\title{
2
}

\section{Web-Enabled Knowledge-Intensive Support Framework for Collaborative Design of MEMS*}

\author{
Xuan F Zha \\ National Institute of Standards and Technology, USA
}

\begin{abstract}
Micro-Electro-Mechanical Systems (MEMS) design and manufacturing are inherently multi-physical and multi-disciplinary; no single person is able to perform a full development process for a MEM device or system. This chapter presents a web-enabled design platform for collaborative design of MEMS. The proposed web-based distributed object modeling and evaluation framework with client-knowledge server architecture, KS-DMME, allows multi-users/designers in different locations to participate in the same design process. Under this framework, concurrent integrated MEMS design and simulation models can be built using both local and distributed resources, and the design collaboration can be realized by exchanging services between modules based upon CORBA standard communication protocol. To facilitate the rapid construction of the concurrent integrated design models for MEMS, a prototype web-enabled design system, Web-MEMS Designer, is implemented through concurrent integration of multiple distributed and cooperative knowledge sources and software. By use of the developed prototype system, MEMS design and simulation can be carried out simultaneously and intelligently in an integrated but open design environment on the web. A case study of a microgripper design for micro robotic assembly systems is provided to illustrate how designers in different teams and organizations may participate and collaborate in MEMS design.
\end{abstract}

\footnotetext{
* This work was supported by the Post-Doctoral Fellowship Program of Korea Science \& Engineering Foundation and the Korea Research Foundation grant funded by the Korean government (MOEHRD).
} 
Keywords: MEMS, World Wide Web, network-centric design, design with objects, distributed module modeling and evaluation (DMME), knowledge-based decision support systems, integrated design and analysis, collaborative design

\section{INTRODUCTION}

Micro-electrical Mechanical Systems (MEMS) are systems that generally incorporate silicon based electrical and mechanical elements at the micro level $\left(10^{-6} \mathrm{~m}\right)$. It is estimated that the MEMS market has already reached around \$30 billion. Micro-Electro-Mechanical Systems (MEMS) is a rapidly expanding field of multi-disciplinary technology, which takes advantage of semiconductor fabrication processes to produce micron-scale mechanical, fluidic, electric, optical, and other devices. MEMS devices are often integrated with microelectronic circuits, which control their behavior, perform signal processing and computing, and control/activate the behavior of the mechanical structures.

With the parallel development of new technologies, new device configurations, and new applications for microsensors, microactuators, and micro-systems, there has arisen a growing need for multi-disciplinary CAD support for MEMS. Needs, key issues and requirements in this arena have been identified, formulated, and reviewed in [39, 46, 47]. MEMS CAD shares some common techniques with the conventional CAD, but it is different in many ways. The multi-dimensional, multi-disciplinary, and multi-scale nature of MEMS makes the CAD software very difficult to develop [15]. Smart product design can be achieved with the aid of concurrent and intelligent concepts to facilitate design tasks. The inherently multi-physical and multi-disciplinary MEMS design problem requires new concurrent intelligent design methodologies and systems involving the integration of modeling, design, analysis and evaluation, and simulation for MEMS devices or systems as early as possible in the course of the different life-cycle phases. On the other hand, contemporary MEMS design problems often embody significant levels of complexities that make it unlikely that a single designer can work alone. The continuing growth of knowledge and supporting information and ever increasing complexity of design problems has led to increasing specialization. It has been recognized that further rapid progress in MEMS technology will be difficult to accomplish without a full range of multi-level hierarchical design tools (from a high-fidelity device level to a system level). Because of the heterogeneous structure of micro-systems, MEMS design and simulation requires different grades of abstraction and needs the cooperation/ collaboration of different disciplines and resources. Wide-area networks and the internet-based WWW allow users/designers to provide remote design servers. MEMS CAD systems running on these design servers can support a large-scale group of users/designers who communicate with the systems over the network. Based on the web protocols (e.g. HTTP), user/designer interfaces can provide access to the remote Web-based design servers with appropriate web browsers. Users do not need special hardware or software to consult these services. Thus, multiple users/designers in different locations are able to use the same CAD tool and design a MEMS device or system together. With the advent of Internet and WWW, it is expected that one of the focal research areas in MEMS design community will be on the development of web-based design framework/platform for collaborative MEMS design [43]. 
The purpose of this chapter is to present a web-based knowledge intensive development framework to facilitate the rapid construction of concurrent integrated distributed design models for MEMS, and to provide distributed designers with a platform/tool for collaboratively building these models. Specifically, in this chapter, the issues to be addressed include the following:

(1) To explore a new concurrent intelligent design methodology, involving the integration of modeling, design, analysis and evaluation, and simulation, for MEMS devices or systems;

(2) To develop a concurrent knowledge intensive design framework for MEMS design and simulation; and

(3) To develop a distributed intelligent platform for MEMS design and simulation using Java and CORBA over the web.

The structure of the chapter is as follows. It begins with an overview and requirements for network-centric design tools (Section 2). Then, in Sections 3 and 4, the web-based framework for supporting different types of collaborative design activities in a distributed design environment is developed for MEMS design, analysis and simulation. Section 5 provides a detailed implementation of the collaborative MEMS design system. A case study of collaborative microgripper design is provided in Section 6 to illustrate how designers in different teams and organizations may participate in the design of a microgripper for micro-robotic assembly system. Section 7 summarizes the chapter.

\section{CURRENT STATUS OF RESEARCH}

\subsection{Computer-Aided MEMS Design}

MEMS development is a broad field that combines a large range of technical disciplines. Computer-aided design (CAD) tools are clearly needed to reduce the consumption of development resources, and frequently help provide insight into complex physical processes for the evolution of high aspect ratio micromechanical devices (like pumps, valves, and micromotors) as high performance demands are placed on these devices, especially in precision and accuracy. Software modeling tools are rapidly gained acceptance by the design community whenever /wherever they are applicable and useful. CAD tools permit the rational design of these devices and evaluate the effects of parameters such as temperature, strain, acceleration, etc. [32]. Without CAD tools, fabrication remains in the domain of experts, and evolution of the design process relies on empirical approaches. In general, the CAD software packages are structured as sketched in Figure 1, with the design aids used to create the design, simulation to develop the technology, and verification to check the design. The final verification is to avoid wasteful and slow experiments by carrying out less costly computer work in order to get the fabrication right the first time [30].

Several CAD systems, which might facilitate the wider acceptance of MEMS, are discussed below. According to Senturia and Howe [45], the ideal suites of CAD tools required for MEMS development are [49]:

(1) Rapid construction and visualization of three-dimensional solid models;

(2) A comprehensive database of materials properties; 


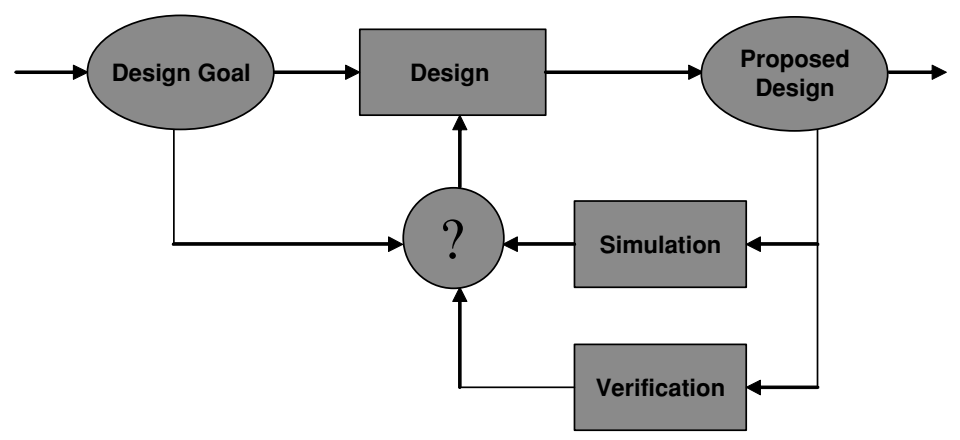

FIGURE 1. Design, simulation and verification in CAD systems

(3) Simulation tools for basic physical phenomena, e.g., thermal analysis, mechanical and structural analysis, electrostatic analysis, magneto static analysis, and fluid analysis;

(4) Coupled force simulators, e.g., thermally induced deformation, electrostatic and magnetostatic actuators, and interaction of fluids with deformable structures;

(5) Formulation and use of macromodels, e.g., lumped mechanical equivalents for complex structures, equivalent electric circuit of a resonant sensor, and feedback representation for coupled-force problems;

(6) Process simulation or process database, including, lithographic and etch process biases; and process tolerances on thicknesses, lateral dimensions, doping, and resistivity levels;

(7) Design optimization and sensitivity analysis, e.g., variation of device sizing to optimize performance, and analysis of effects of process tolerances;

(8) Mask layout;

(9) Design verification, including, construction of a three-dimensional solid model of design using the actual masks and process sequence, checking the design for violation of any design rules imposed by the process, simulation of the expected performance of the design including the construction of macromodels of performance usable in circuit simulators to assess overall system performance;

Several worldwide projects are continuing to develop comprehensive MEMS design tools focusing on either device or system level CAD. They are derived either from the existing microelectronic design tools (ECAD/TCAD) or mechanical tools [14]. Such systems are at boundary between two large CAD industries: electronic design automation (EDA) and mechanical design automation (MDA). Thus, the major task of MEMS CAD systems is to intentionally integrate tools from MDA and EDA. Several vendors, including Coventor, ANSYS, ISE, and CFD Research Corp., are developing MEMS CAD software system. Some examples of MEMS CAD programs under development and developed so far are Oyster [24], CAEMEMS [7], MIT's MEMCAD (now CoventorWare) [13, 14], SESES [25], and IntelliSense [31], IntelliCAD, MEMSCAP, CyberCAD [57], An's MEMS CAD (2000), etc. Oyster facilitates the construction of a three-dimensional polyhedral-based solid mask set and gives a rudimentary process description. MEMCAD is directed at conceptual 

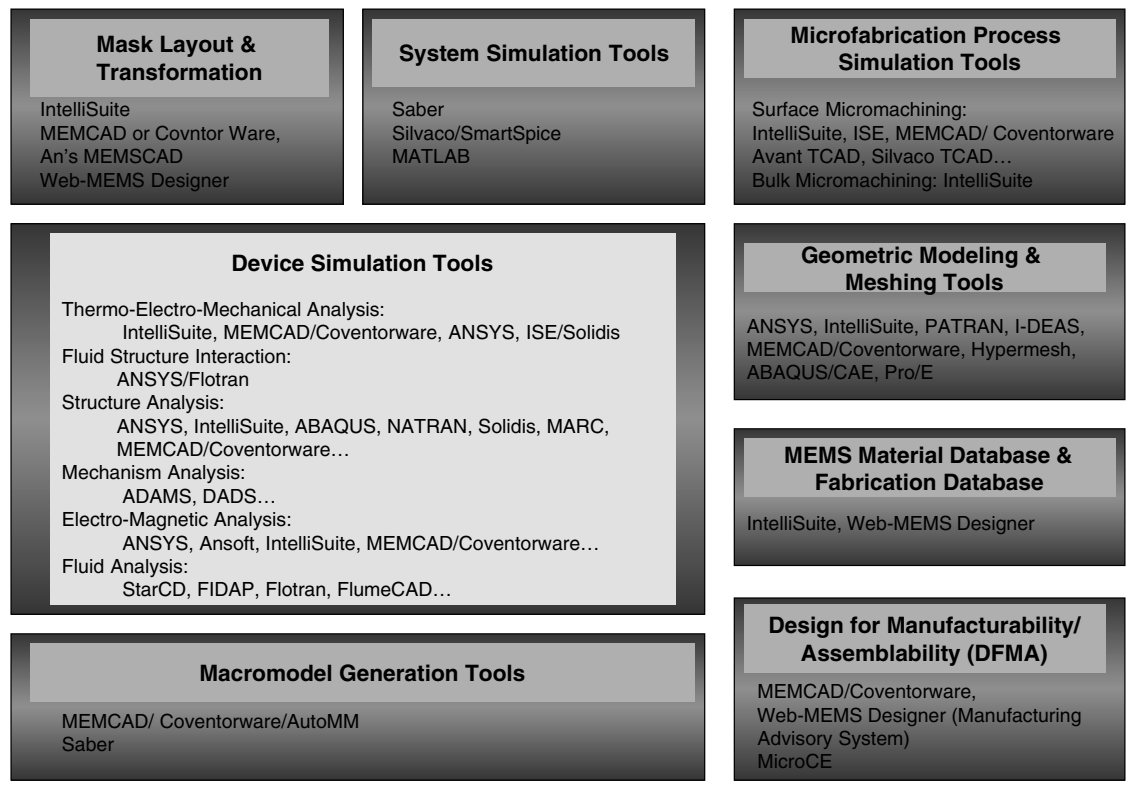

FIGURE 2. Overview of existing MEMS CAD and simulation tools

design and simulation, as well as design verification. CAEMEMS is geared towards design optimization and sensitivity analysis. SESES addresses conceptual design and simulation and design verification. IntelliCAD includes the material database. The material database contains electrical, mechanical, optical, and physical properties of semiconductor thin films collected from the literature. On a general remark, these tools focus on structure design and indeed have little about design that links functions to structures - a notion called synthesis. Moreover, these tools have not provided design process management. To make the CAD system more flexible, a knowledge base/database system is required that has a very systematic representation with less data and more information and that keeps on updating itself as the new information arrives. MEMS CAD is a free MEMS Layout Design tool under development with Java. Since it is programmed by Java, it works on all platforms, such as MS Windows, Linux, Sun, HP and SGI. Its basic functions are: 1) photo mask design, 2) mouse-driven drawing, 3) command-line free polyline drawing, 4) CIF data conversion with polyline, and 5) 3D exploded view with mouse/keyboard navigation. Figure 2 gives an overview of existing MEMS CAD and simulation tools.

In the general area of design, the development of intelligent computer support systems for design has been the subject for many years. One of the key technologies is relevant to the modeling of functions and structures. The matured technology is available for modeling functions alone, but neither the function nor the linkage of the function to structure is available. The notable modeling idea is the so-called function-behaviour-structure framework. This framework is not yet unified, which leads to ad-hoc developments of systems for MEMS design. Contemporary CAD tools for MEMS have not considered the process management, which puts a high demand for research on this missing component. 


\subsection{Collaborative Design for MEMS}

There have been many research efforts on enabling technologies or infrastructure to assist product designers in the computer network-centric distributed design environment [11, $17,28,35,36,40,50,59,64]$. Some of them are intended to help designers to collaborate or coordinate by sharing product information and manufacturing services through formal or informal interactions $[8,26,51]$. Others propose formalized frameworks that manage conflicts between design constraints and assist designers in making decisions [8, 37, 38, 42]. There are also national-level efforts involving university and industry collaboration to make a variety of engineering services available over the Internet [29]. The RaDEO program is concerned with comprehensive information modeling and design tools needed to support the rapid design of electro-mechanical systems. It supports engineers by improving their ability to explore, generate, track, store, and analyze design alternatives.

The SHARE project by Toye et al [59] supports design teams by allowing them to gather, organize, re-access and communicate design information over computer networks to establish a shared understanding of the design and development process. While SHARE is primarily directed towards interaction through integrated multimedia communication and groupware tools, the NEXT-LINK project incorporates agents to coordinate design decisions affected by specifications and constraints [40]. A network-centric design system using interacting agents to integrate manufacturing services available over the network is under development [11].

The Electronic Design Notebook (EDN) is an interactive electronic document that maintains the look and feel of an engineering document to provide an integrated user interface for computer programs, design studies, planning documents, and databases [26]. Manufacturing tools and services are encapsulated in the hypertext documents and distributed through servers using HTTP [51].

A computer-based design system developed by Sriram et al $[52,53]$ provides a shared workspace where multiple designers work in separate engineering discipline. In their Distributed and Integrated Environment for Computer-aided Engineering (DICE) program, an object-oriented database management system with a global control mechanism is utilized to resolve coordination and communication problems. Design rationale provided during the product design process is also used for resolving design conflicts.

A design information system proposed by Bliznakov et al $[5,6]$ incorporates a hybrid model for the representation of design information at several levels of formalization and granularity. It is intended to allow designers in a large virtual organization to indicate the status of tasks assigned to each designer or team so that other designers can follow their progress. A central database manages pointers and access methods for product and process information in the distributed environment.

Hardwick and Spooner [17] propose an information infrastructure architecture that enhances collaboration between design and manufacturing firm. This architecture uses the WWW for information sharing and the STEP standard [33] for product modeling. It utilizes the CORBA standard for interoperability between software applications in the virtual enterprise.

$\mathrm{N}$-dim is a computer-based collaborative design environment for capturing, organizing and sharing data [60]. It is a base, on which applications can be added for the purpose of history maintenance, access control and revision management. The primary focus of 
environment is on information modeling. The system provides a way for defining information types that capture the relations between data or models.

Pahng et al [35, 36] developed a Web-based framework for collaborative design modeling and decision support, based on the distributed object modeling and evaluation (DOME). The DOME framework asserts that multidisciplinary problems are decomposed into modular sub-problems. Modularity divides overall complexity and distributes knowledge and responsibility amongst designers. It also facilitates the reuse of modeling elements. Thus, DOME allows designers to define mathematical models or modules and integrate or interconnect them to form large system models. In DOME, a multiple attribute decision method is used to capture preferences and evaluate design alternatives from different viewpoints.

The above on-going research efforts pave the ways in which a network-centric design environment is able to support product designers and suggest what a computer-based design tool or system should look like in such an environment. However, they do not provide a structured and formalized framework for modeling the characteristics of multi-disciplinary and multi-objective design problems, and none of them are focused on the network-centric, distributed and collaborative design of MEMS. Existing CAD tools above for MEMS design, simulation and manufacturing are unable to support collaborative MEMS modeling and design activities. They are generally specialized and stand-alone applications. It is very difficult to use them for understanding and designing the integrated performance of product systems. Therefore, they are unable to support and coordinate highly distributed and decentralized MEMS modeling and design activities [72]. The motivation and vision presented in this chapter share some similar themes with [28, 35, 36] but emphasizes design and simulation modeling, decision-making, and search/optimization for MEMS.

\section{KNOWLEDGE INTENSIVE COLLABORATIVE FRAMEWORK FOR NETWORK-CENTRIC DESIGN}

Contemporary design process is knowledge-intensive and collaborative. The knowledge-intensive support become critical in the design process and has been recognized as a key solution towards future competitive advantages in product development. The integrated design requires the skills of many design designers and experts that each participant creates models and tools to provide information or simulation services to other participants given appropriate input information. It is the goal that the collective network of participants exchanging services forms a concurrent model of the integrated design.

Based on the DOME framework [35, 36], a web knowledge server framework, was developed for collaborative design process $[66-69,72]$. The developed knowledge-intensive framework adopts the design-with-objects [66, 70], module network $[4,35,36]$, and knowledge server paradigms [10]. The knowledge server paradigms are techniques by which knowledge-based systems can utilize the connectivity provided by the Internet to increase the size of the user base whilst minimizing distribution and maintenance overheads. The knowledge intensive system can then exploit the modularity of knowledge-based systems, in that the inference engine and knowledge bases are located on a server computer and the user interface is exported on demand to client computers via network connections. Thus, design 


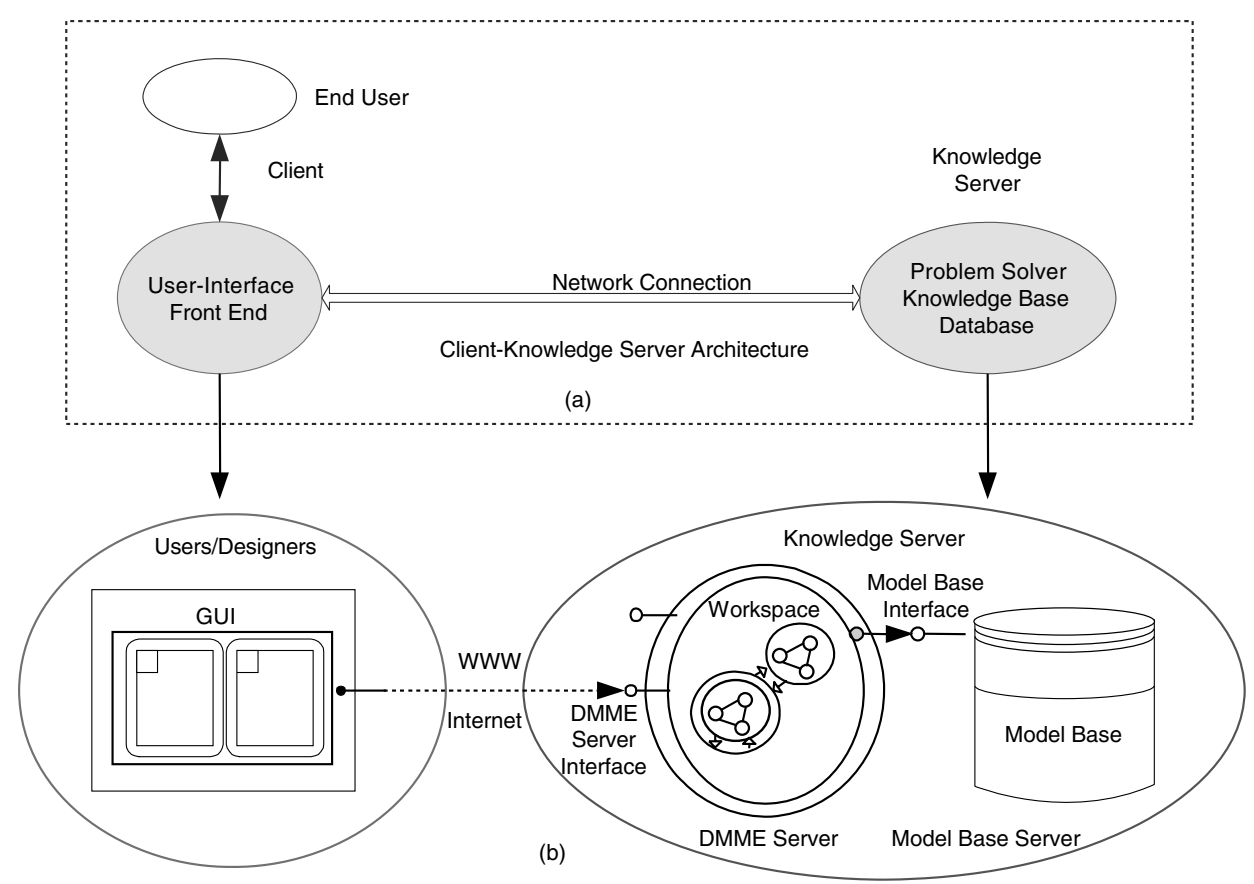

FIGURE 3. (a) Client-knowledge server architecture and (b) main components for KS-DMME

modules or objects are connected together so that they can exchange services to form large integrated system models. The module structure leads itself to a client (browser)/knowledge server-oriented architecture using distributed object technology. The main system components of the developed client/server architecture are shown in Figure 3. Each of these components interacts with one another using a communication protocol (CORBA) over the Internet so that it is not required to maintain the elements on a single machine. As a gateway for providing services, the interface of a system component invokes the necessary actions to provide requested services. To request a service, a system component must have an interface pointer to the desired interface.

With a client / knowledge server architecture, the characteristics of the knowledge intensive framework may be described as follows: (i) extensive knowledge based; (ii) hybrid intelligent system that integrates various knowledge sources; and (iii) concurrent, integrated, distributed. Thus, an intensive knowledge model for design is a large-scale knowledge framework that allows processing various types, different levels, and multiple functionality of knowledge in a design process [66].

\section{KS-WebDMME FRAMEWORK FOR COLLABORATIVE DESIGN OF MEMS}

The development process of MEMS devices or systems includes many steps from design, analysis and simulation, fabrication, to assembly/package and operation. Each of which is difficult, high-cost and time-consuming. Many loops of these steps are needed in the development process. In this section, we will discuss how the KS-WebDMME framework above is used for network-centric MEMS design, analysis and simulation process. 


\subsection{MEMS Design Process and Environment}

A wide range of design problems are included in MEM devices or systems development, such as conceptual design, configuration design, process simulation, solid body geometric renderings from photo-masks and process descriptions, optimization of geometry and process sequence, micro assembly design, planning and simulation, and design of full systems. There are generally two rather different types of CAD requirements [47]: conceptual design phase and product-level phase. The first conceptual phase of a new device is to assist in finding practical configurations; the second product-level phase is to enable careful attention to physical behavior and parasitic phenomena. There is a great benefit if the actual device masks and process description can be used as input to the simulations. The rendering of three-dimensional solid models from mask and process data, both to permit checking of geometries and as input to physical simulation, assures that the device being simulated is also the one being built.

MEMS CAD can be categorized into the work at the following levels: system, device, physical/behavioral, and process level, in which lumped networks, energy-based macromodels, 3D simulation, and TCAD are included respectively ([47, 61, 62], MEMCAD 2000). The host of modeling and simulation requirements for a MEMS CAD system at these levels can be identified and described as follows:

(1) process modeling tools for all process steps;

(2) process optimization tools to achieve a desired device geometry (e.g., topology optimization);

(3) physical simulation in multiple coupled energy domain;

(4) construction of designer-useful behavioral models from simulation (micro models);

(5) device optimization tools to achieve desired device behavior;

(6) insertion of behavioral device models into system-level simulation tools;

(7) behavioral model optimization for desired system performance

In an ideal MEMS design environment, the user will first simulate the fabrication process steps to generate the 3D geometrical model including fabrication dependent material properties and initial conditions (e.g. fabrication induced stresses). The input to this simulation step is the mask layouts (e.g. in CIF or GDS II format) and a process description file (e.g. PFR). To compute fabrication dependent initial fields, the initial geometry model will be meshed and physics based process models (deposition, etching, milling, bonding, annealing, etc) will create a simulation-ready virtual model with complete definition of material properties, boundary and volume conditions, and physical/numerical parameters for field solvers. All model parameters should be specified directly "on geometry" rather than on mesh to allow multi resolution (grid independence) and solution-based mesh adaptation. The ultimate goal, of course, is that the device and the associated system are fabricated, and the system performance is as desired. To the extent that issues can be anticipated through simulation and modeling, also called computational prototyping, costly fabrication experiments can be reduced in number and increased in effectiveness. Figure 4 shows MEMS design methodology and modeling levels.

\subsection{Web-Based Collaborative Design Platform for MEMS}

Based on the design process of MEMS, the KS-DMME architecture for distributed collaborative MEMS design can be illustrated as shown in Figure 5. Under this framework, 

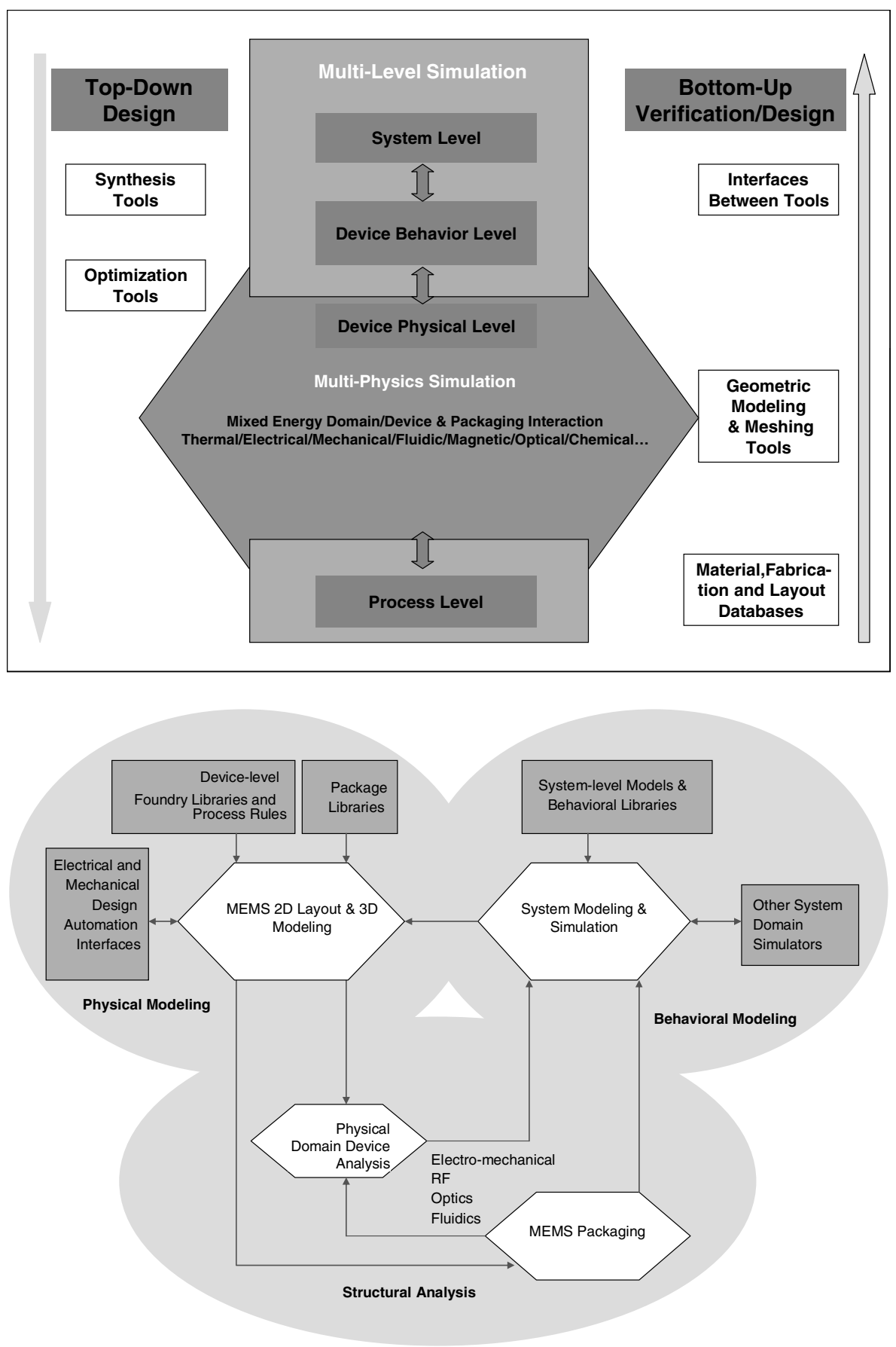

FIGURE 4. MEMS design methodology and modeling levels 


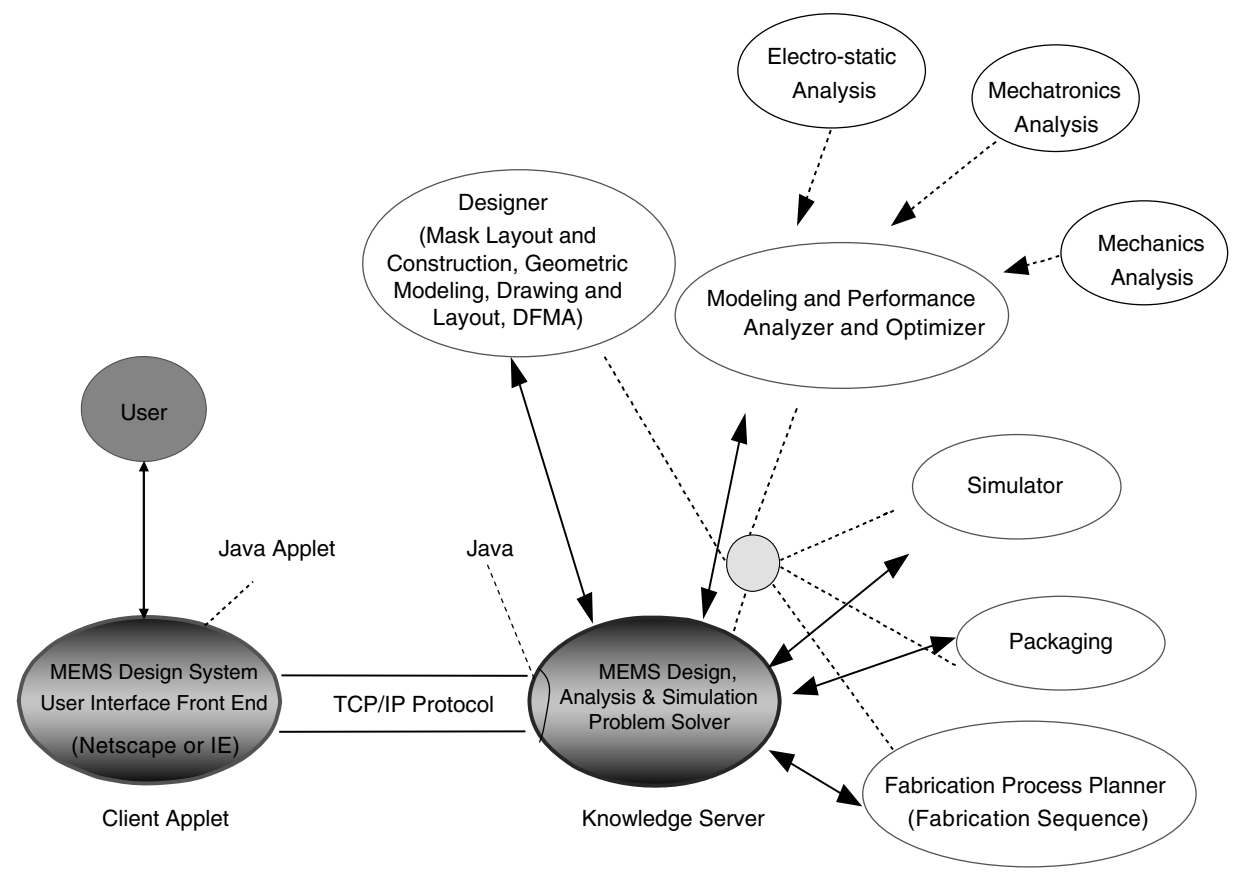

FIGURE 5. KS-DMME architecture for MEMS design, analysis and simulation

the requirements for the Web-based MEMS development tool can range from complex intelligent design, modeling, and simulation capabilities to more narrowly defined requirements. Its capabilities should be built into selectable or configurable, and knowledgeintensive modules that are packaged together to meet the requirements of a desired development flow.

The web-based collaborative MEMS design platform should be able to address the following issues: device layout and construction; device modeling and simulation; system modeling and simulation, and package, etc. The device layout and construction suite includes a direct, automatic connection between design of process and layout and full 3D-device modeling and visualization. It enables MEMS design to be driven by either experienced layout designers or mechanical engineers demanding full 3D editing capabilities. The device modeling and simulation suite provides solvers for the specific 3D physics of each kind of MEMS device. Specific knowledge on MEMS device modeling will be wrapped around state-of-the-art hybrid finite element and boundary element numerical tools. Thus, MEMS designers do not have to be experts in numerical techniques to get usable, accurate simulation results. The system modeling and simulation suite provides tools to help the designer understand manufacturing sensitivities and co-design of MEMS systems and devices. Design engineers can build and simulate accurate system models containing MEMS components integrated with external or on-chip circuit systems. Advanced tools enable automatic extraction of efficient, physically realistic SPICE and SPICE-like models of MEMS components from three-dimensional analysis. The packaging suite provides MEMS designers and packaging groups with tools to support communication and co-design. It enables 
true-coupled 3D package and device co-simulation. Package and MEMS groups can communicate by sharing quantitative models of package induced effects, along with tools to understand detailed device sensitivities to package design variables.

The solution to providing distributed MEMS design support in this research is to extend an original stand-alone MEMS design system, i.e., MEMS Designer [66], into a Web-based collaborative MEMS design system, i.e., Web-MEMS Designer. The system is deployed on a web server enabling access via the Internet a comprehensive suite of scalable and configurable software tools for MEMS design and simulation. Details about the implementation of the Web-based MEMS Designer system will be discussed below.

\section{DEVELOPMENT OF WEB-BASED COLLABORATIVE MEMS DESIGN SYSTEM}

To facilitate the rapid construction of the concurrent integrated models, a web-based collaborative design environment is essential for MEMS design and simulation. In this section, we describe the implementation of the prototype collaborative MEMS design system, Web-MEMS Designer. The focus is on the description of the technologies employed in the design and development of the Web-MEMS Designer system under the KS-DMME framework discussed above.

\subsection{System Overview}

The MEMS Designer system is a knowledge-driven design platform that delivers complete end-to-end development flow for MEMS-enabled devices or systems. It equips design engineers with the means to develop MEMS devices or systems from an initial concept through complete coupled analysis, which can also include package design characteristics, and ultimately extract high-level models for system simulation. The Web-MEMS Designer system exploits the modularity of knowledge-based systems, in that the inference engine and knowledge bases are located on server computers and the user interfaces are exported on demand to client computers via the Web. It is therefore a distributed intelligent development environment, consisting of 3D design, modeling and simulation software tools, which enable the creation of complex micro and/or MEM devices. The design flow of the MEMS Designer system is similar to MEMCAD that coordinates four key MEMS product development functions: layout and construction, device modeling, systems modeling and packaging analysis. The developed prototype MEMS Designer system contains a set of modules that are able to preliminarily support some of these functions, as follows:

(1) function-behavior-structure modeler for conceptual MEMS design (MEMS Designer @ Concept) (Appdendix B);

(2) 2D drawing tool (including layout editor) (MEMS Designer @ Concept);

(3) masking and fabrication process sequence builder (MEMS Designer@Builder);

(4) embedded manufacturing process and material databases (MEMS Designer @ Builder (MaskProcess));

(5) 3D geometric modeler and viewer (MEMS Designer @ Builder);

(6) manufacturing advisory system (MEMS Designer @ Advisor);

(7) embedded design optimization tool (e.g. GA tools) (MEMS Designer @ Analysis);

(8) 2D and 3D FEM analysis (including an ANSYS interface) (MEMS Designer @ Analysis). 
The capabilities of these modules enable the MEMS Designer system to offer a special design platform for collaborative MEMS design, analysis and simulation.

\subsection{System Implementation}

The implementation of the prototype Web-MEMS Designer system is actually a threestage process. The first stage was to convert MEMS Designer into a stand-alone application [66], involving the translation of the original knowledge base into an appropriate format and reconstructing the necessary functionality. The second stage was to convert the standalone application implemented in $\mathrm{C} / \mathrm{C}++$ into $\mathrm{CGI}$ executables that were deployed on a standard Web server, in terms of template Web pages to contain dynamically generated input forms, the necessary code to extract knowledge from submitted forms, and display results. The third stage was to implement the Web-MEMS Designer using Java and CORBA technologies integrating with a Java Expert System Shell, Jess/FuzzyJess, based upon Windows NT-based environment with a front-end Web-browser-based Graphical User Interface (GUI). Jess is a multi-paradigm programming language that provides support for rule-based, object-oriented, and procedural programming system language. The underlying modules are written in Java/Java3D/JDBC, respectively. The implementation architecture shown in Figure 6 uses the two-tier client/ knowledge server architecture (Figure 3) to support collaborative design interactions. Designers can integrate MEMS design problem models with the existing application packages, such as Java3D and JDBC for CAD and database applications.

The CORBA [48] standard is used to add distributed communications capabilities to modules (Orbix and OrbixWeb from IONA Technologies Ltd [20]). CORBA serves as an

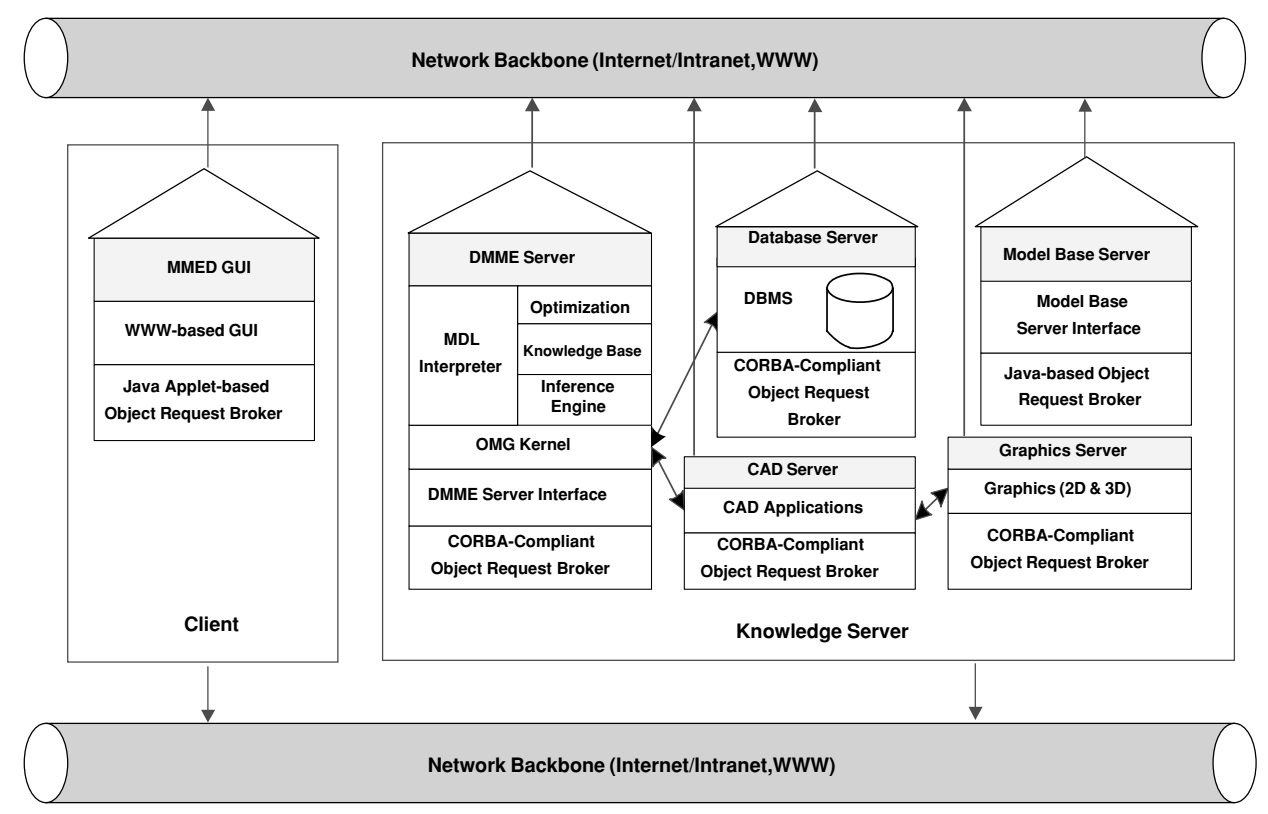

FIGURE 6. Implementation of the open design environment 


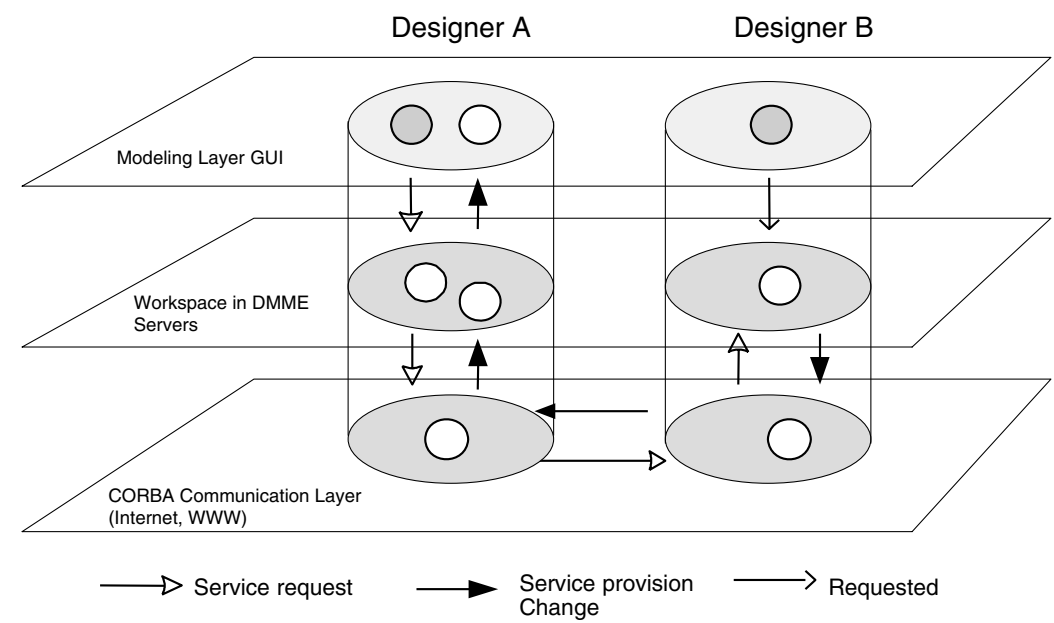

FIGURE 7. Service exchanges between distributed modules

information and service exchange infrastructure above the computer network layer and provides the capability to interact with existing $\mathrm{CAD}$ applications and database management systems through other Object Request Brokers (ORB). In turn, the KS-DMME framework provides the methods and interfaces needed for the interaction with other modules in the networked environment. These interactions are graphically depicted in Figure 7. When Designer B makes a change, the service corresponding to the request from Designer A will reflect the design change. The enumerated request shows the sequence for Designer A needs obtaining the service that. The light gray module seen by Designer A is the remote module published by Designer B. The underlying collaboration mechanism is based on the board systems. Each modular system has two-board systems, black and white board, as shown in Figure 8. The blackboard system is used for the local modular system to store intermediate reasoning and calculation results. It dynamically flushes in running. The whiteboard system is used for collaboration, which is actually a bulletin board system.

The Web-MEMS Designer GUI provides users with the ability to examine the configuration of design problem models, analyze tradeoffs by modifying design parameters within modules, and to search for alternatives using an optimization tool. The GUI is a pure client of the DMME server, delegating all events to an associated DMME server. For wide accessibility and interoperability, the GUI is implemented as a Web browser-based client application, which is a combination of HTML/XML documents and Java applets. For the CORBA-based remote communication between the GUI Java applets and the back-end side system components such as DMME server, CAD server, graphics server, and model base server, a commercial ORB implementation of Java applets (OrbixWeb) is employed [20]. Based on the system implementation architecture in Figure 6, the functionality of the knowledge server is achieved through implementing DMME servers, model base server, core knowledge engine, database server, and even knowledge base assistant and inter-server communications explanation facilities. Figure 9 shows several demonstration screenshots of 


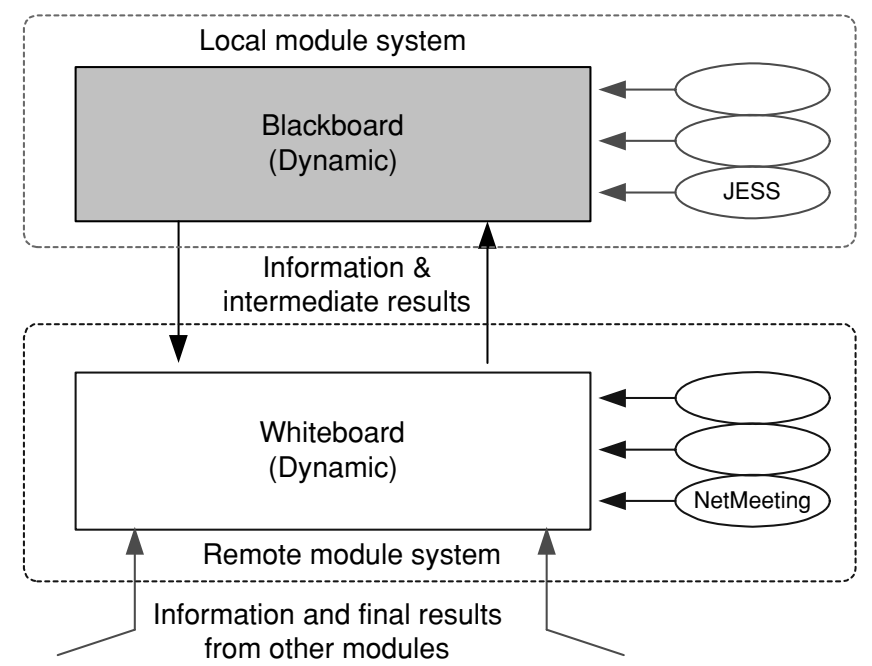

FIGURE 8. Blackboard and whiteboard system for collaboration

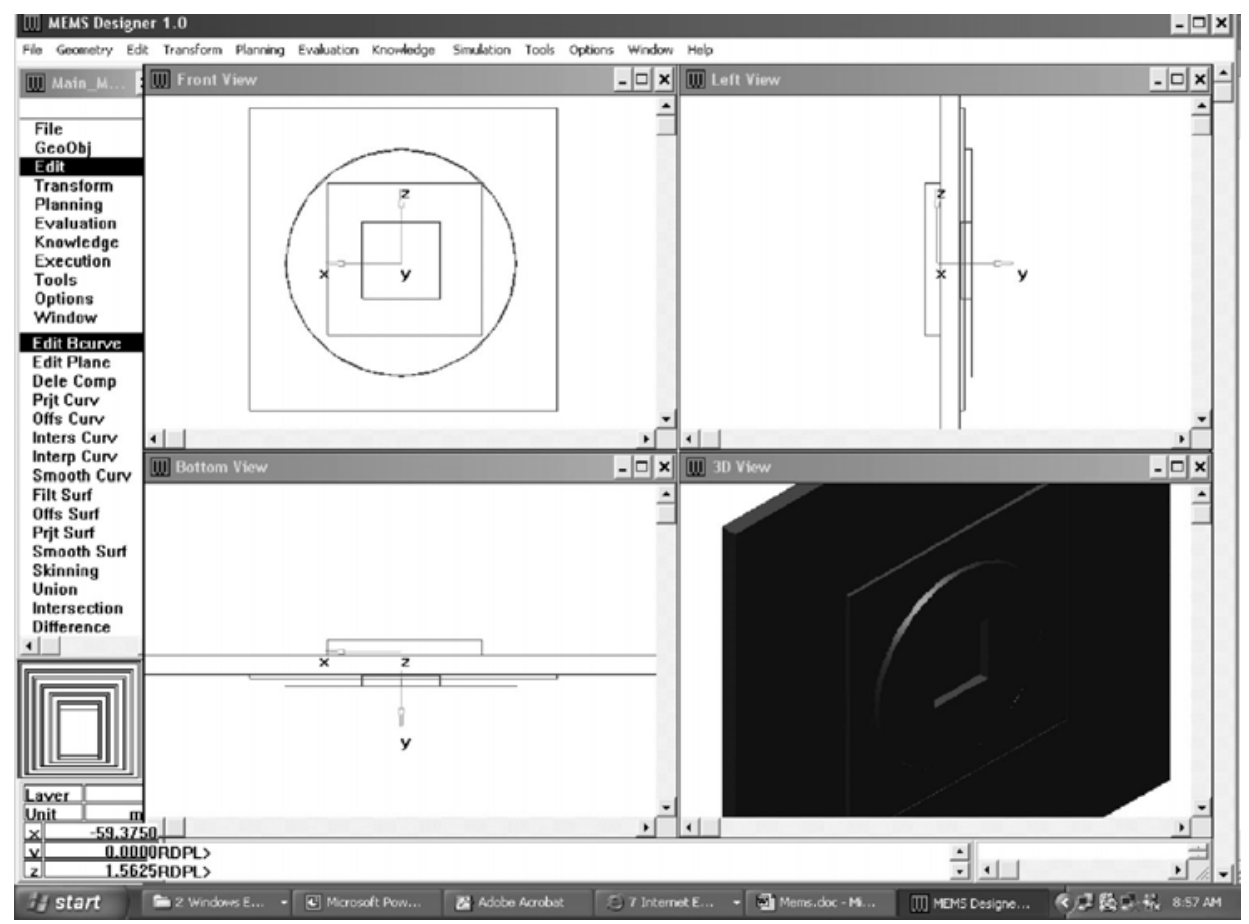

FIGURE 9. MEMS Designer GUI: Standalone and Web Enabled. (a) C++ MEMS Designer Standalone (Flow Rate Sensor Demo) 


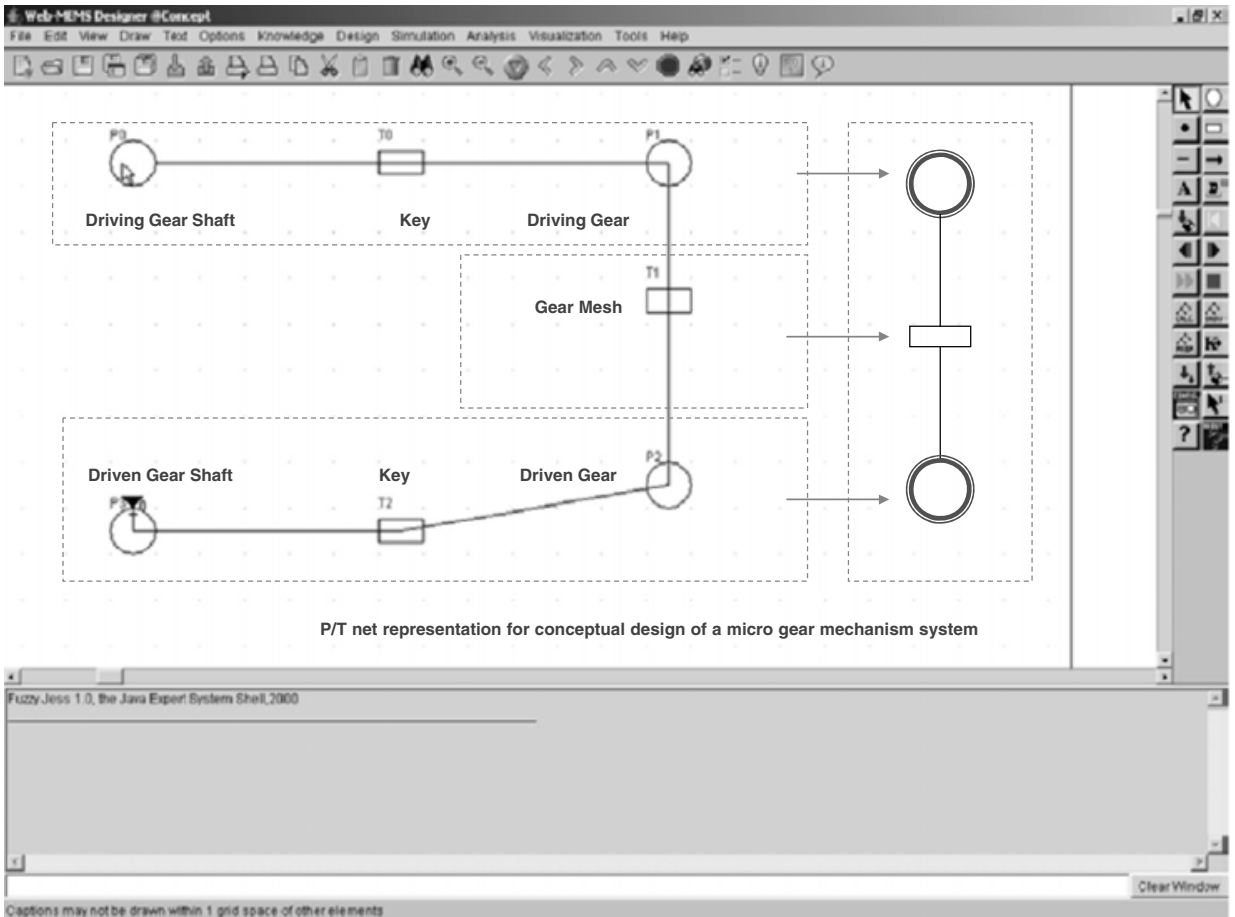

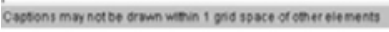

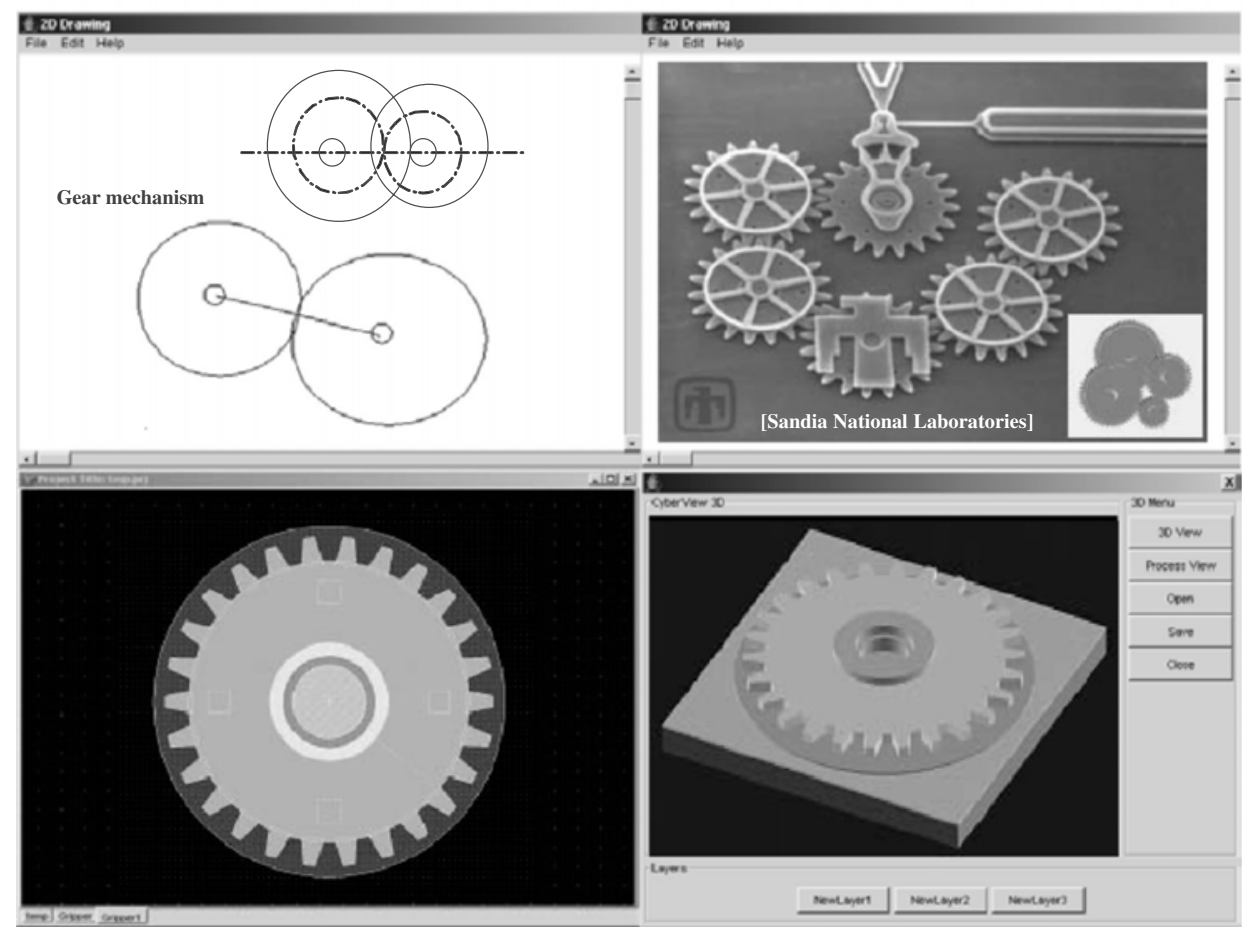

FIGURE 9b. Java Web-Enabled MEMS Designer @Concept GUI (Gear Mechanism Demo) 

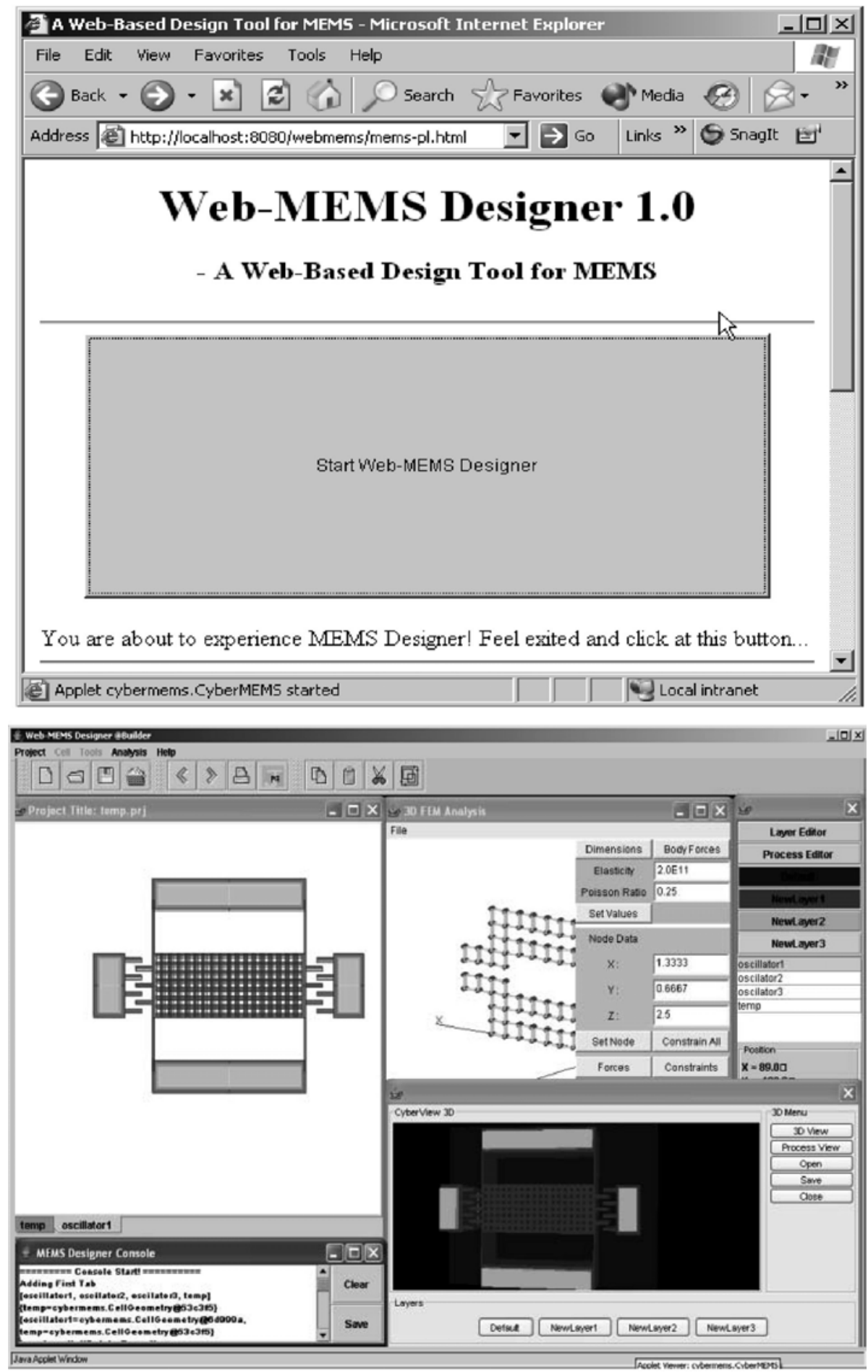

FIGURE 9c. Java Web-Enabled MEMS Designer @Builder GUI (Demo) 
MEMS Designer GUI both as a standalone and as an applet. Figure 9(a) is a design screenshot of flow rate sensor using C++ MEMS Designer standalone; Figure 9(b) illustrates the conceptual design of micro gear mechanism using the MEMS Designer @ Concept; Figure 9(c) gives screenshots of Java-based web-enabled MEMS Designer startup and design for an oscillator by Web-MEMS Designer @ Builder.

The GUI interacts with designers' events and requests to the DMME server that provides the back-end implementation for the modeling of design problems. The core of the server is based upon object modeling and evaluation (OME) kernel $[35,36]$ written in Java/Java3D/JDBC, integrating Jess/FuzzyJess. The back-end implementation for knowledge server, including DMME server, and model base server, and the front-end interface to the GUI are written in Java. The DMME server manages each design session in a workspace and can simultaneously maintain several workspaces. The workspace manages administrative aspects of a model (e.g., ownership, access privilege, links to other workspaces in different DMME servers, etc.). The DMME server itself is a CORBA-compliant distributed object and can communicate with other DMME servers. The model base server maintains persistent storage for models created by the DMME servers. The model repository stores a model in a model definition file (MDF) with two parts: meta definition and model definition. The meta definition contains the information such as model id, ownership and access privilege information. The model definition is based upon a model definition language (MDL) used by the system. The core knowledge engine includes knowledge base and problemsolving paradigm (inference engine). The knowledge base is built in Java/Jess. The Web database system is developed by use of Microsoft Access or MySQL databases to store the details of data and Java programs to access these databases through JDBC connections $[58,68]$.

To enable real-time communication/talking among customers, designers and manufacturing engineers, Windows NetMeeting is incorporated into KS-DMME and used as a video design conferencing tool. The whiteboard system is implemented in NetMeeting. Using the chat function of NetMeeting, designers/ users can talk to and discuss with customers, other designers, and manufacturing engineers for any issues. Using the program sharing function of NetMeeting, designers/users can also share the CAD system with manufacturing engineers to discuss or design the product together in real-time. If designers/users share an image editing software, such as the Microsoft Paint, and import the image file of object (module, product variant, family), then they can mark it up, and discuss any issues.

\section{CASE STUDY: COLLABORATIVE DESIGN FOR A MICROGRIPPER}

To illustrate the application of the developed Web-MEMS Designer system for collaborative MEMS design process, a working case of a microgripper design for a micro-robotic assembly system was carried out. The design case originated from $[9,55]$. It was chosen because of its interdisciplinary and developing nature. The research results from this particular case could be generalized to cover other designs that require collaboration and integration of multiple domains. The focus of the illustration is on how designers from different teams, divisions, or companies in remote locations may participate to create an integrated design model for the microgripper design. 


\subsection{Problem Definition}

Currently, the most common technique used to fabricate MEMS devices involves some form of a lithography-based micro-fabrication method with little or no little assembly. MEMS products manufactured utilizing this technique are accelerometers, and inkjet printer heads. However, certain micro systems or parts cannot be manufactured using MEMS techniques. MEMS devices that have incompatible processes, different materials, or complex geometries, have to be 'assembled'. Assembly practices require that a human operator pick and place micro-parts manually using high power microscopes and microtweezers. This method of assembly is tiresome, time consuming, unreliable, and costly. The term 'micro assembly' is used to describe the assembly of micronsized parts that are extremely small (in the order of $10^{-6}$ meters). As manual assembly of micro devices is extremely difficult and tedious, there is a need to design computer-controlled approaches to facilitate rapid assembly. In this context, the design of automated or semi automated environments for micro assembly applications become important. Innovative computer based automated assembly methods must be developed to increase efficiency, reliability, and reduce cost. One of the long-term goals of this research is to develop an integrated physical and virtual assembly system to support the design and analysis of candidate assembly and manipulation. The scope of discussion in this chapter is restricted to the design of the micro robotic assembly work cell to support the assembly of micro devices. The emphasis of discussion is on the distributed collaborative design of microgripper used for micromanipulation/micro assembly.

Suppose that designers from different teams, divisions, or companies in remote locations would like to participate in designing a micro-robotic assembly work cell. The micro robotic assembly work cell discussed consists of four major components: the robot system, the micro-positioning stages (work platform) for work piece, a micro-gripper used for manipulation, micro sized parts to be assembled, and microscope with camera to provide guidance and visual feed back, as illustrated in Figure 10. The microgripper could be used as a tool in micro assembly and micro measurement in the micrometer scale.

We use the developed system to carry out collaborative design of the micro robotic assembly work cell. We first decompose the robotic system design problem into modules and define how these modules are related to one another to create the model of a robotic system design problem. The relationships among these modules specify how outputs of a module are connected to inputs of other modules. Then, we use the distributed module modeling and evaluation (DMME) approach to carry out the distributed collaborative design of micro robotic manipulation systems and microgripper used for micromanipulation under the system support.

\subsection{Collaborative Micro Robotic System Design Process}

Generally, a micro-robotic assembly system consists of three major parts: a micro-robot system, an assembling platform, and micro-components to be assembled. A micro-robot system is generally composed of a micro-robot body and its end-effector with a microgripper. Thus, the overall topology of the design problem and the design workspace can be illustrated in Figure 11. As shown in Figure 11, the micro-robot and microgripper manufacturers provide their design and simulation models to the micro-robot system designers who in turn 


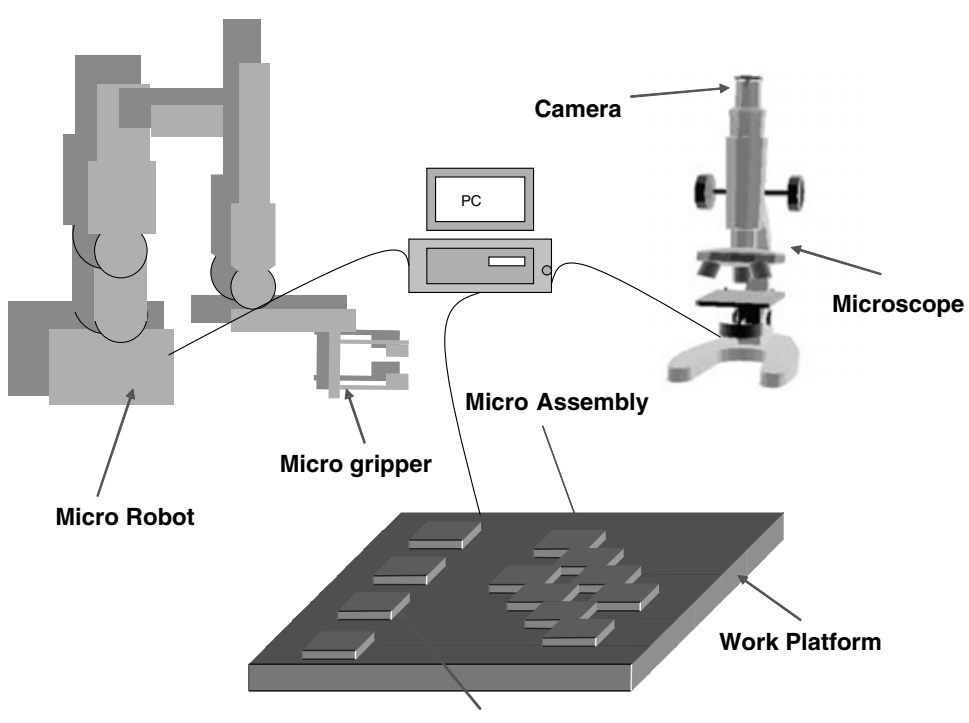

Micro Parts

FIGURE 10. Concept of the micro robotic assembly system

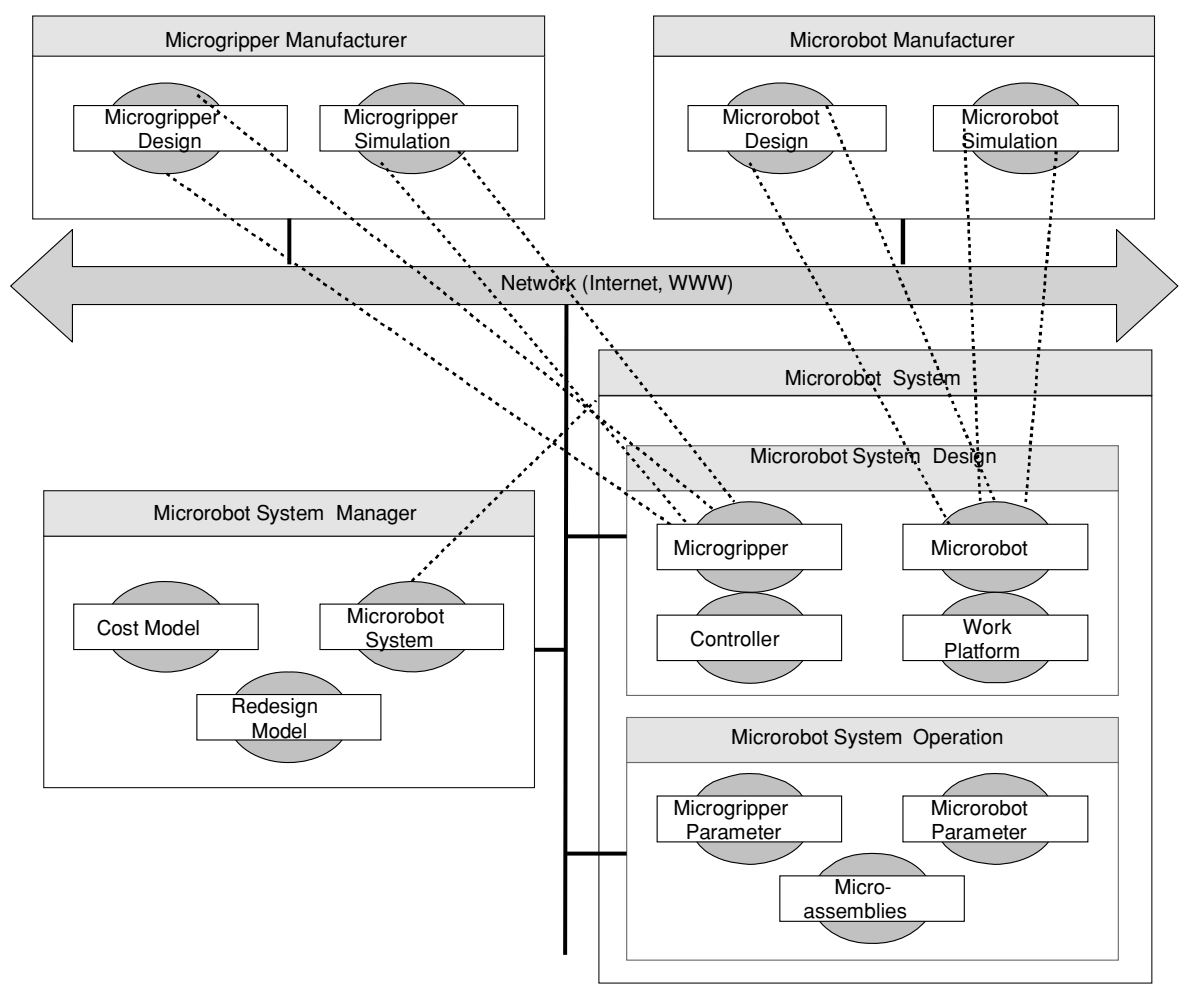

FIGURE 11. Problem topology of the micro-robot system design model 


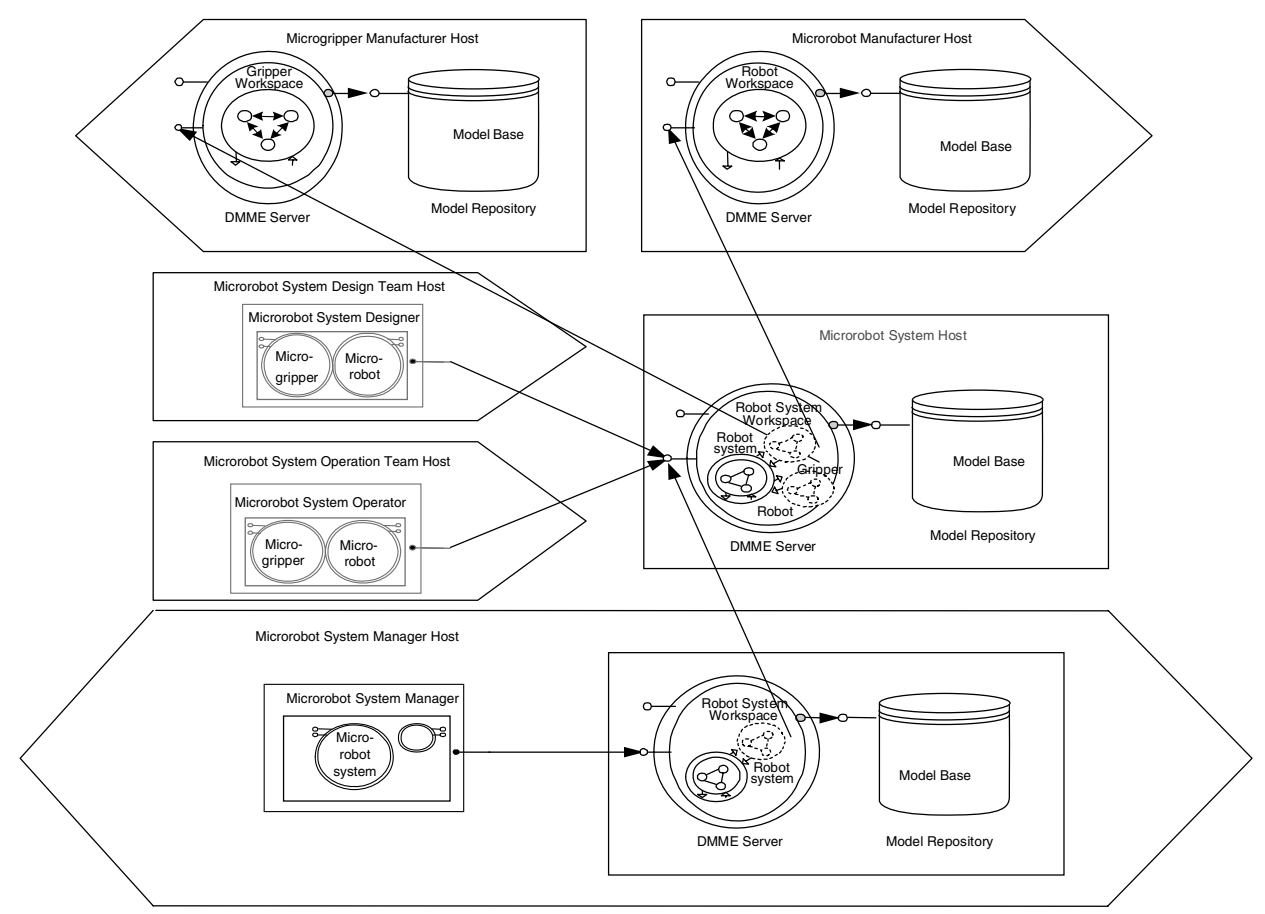

FIGURE 12. Shared design workspace as viewed by the robotic system designers and operator; robotic system manager model connected to the robot system design model

develop the technical models for the micro-robot system. The micro-robot system manager collaborates with the micro-robot system designers and provides models and data for microrobot operating conditions and requirements. Then, he/she uses the micro-robot system design models created by the micro-robot system designers to develop cost evaluation and redesign models. The microgripper and micro-robot manufacturers develop models for their products so that their customers can obtain performance predictions and evaluations for different parametric configurations and operating conditions. These individual models are constructed, published and served by different companies, as shown in Figure 12. If a single designer or company creates all these models and provides all those services the design work is carried out in an individual workspace, as illustrated in Figure 13.

The design session GUI of Web-MEMS Designer creates and depicts the layout and construction and simulation models or modules in the microgripper design workspaces. Designers can use any commercial web browser to access and work on these modules. Since users/customers will connect to these models to assess the performance of their products, designers should decide how to publish these models, i.e., what simulation services the models offer given appropriate input information. When a model is published anyone can use its services if he/she has the appropriate access privileges. The owner of the model can or may want to conceal knowledge intensive engineering formulae or supply chain information embedded in the model. Through service publication, a designer sets access privilege levels for the services of each module in their workspace. Therefore, the designer 


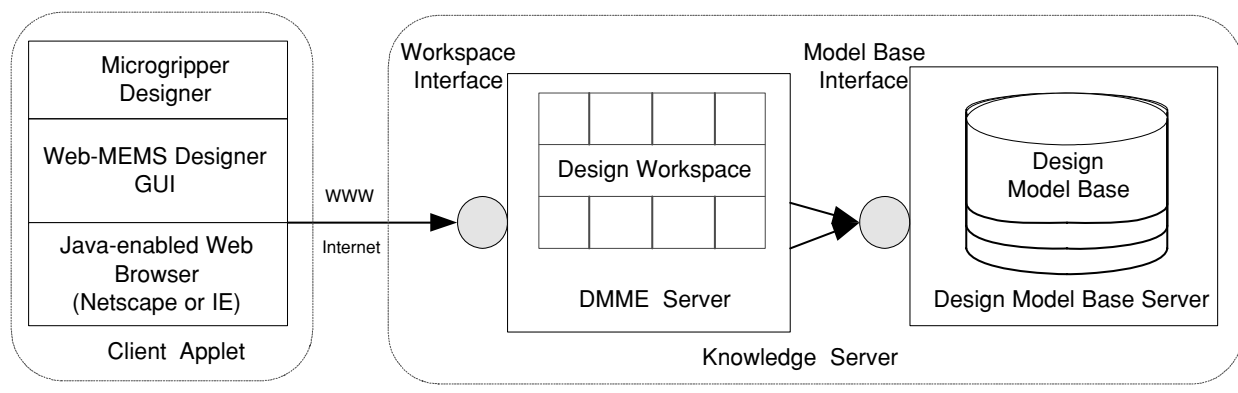

FIGURE 13. Individual workspace for single microgripper designer

working on the design model is assigning access privileges to the services that modules can provide.

As the robot system design and operation are tightly coupled, it would make sense for designers in these groups to share a common model. Thus, while designers from different groups are in remote locations, they can access into the same workspace, which is referred as a shared workspace. Figure 12 shows the design workspace as viewed by the designers from the robot system design team and the robot operation team. The robot system design team is connected to the robot and gripper manufacturing teams so that their robot system design integrated with gripper and robot models can be tested. In this implementation and demonstration, the robotic system is assembled through the use of predefined fixed types of modules (joint modules and link modules) in distributed module inventories (repositories). These modules are published and can be accessed.

Since the micro robot system design and operations are tightly coupled, it would make sense for designers in these groups to share a common model. Therefore, while designers from different groups are in remote locations, they can access into the same workspace, which is referred as a shared workspace. Figure 12 shows the design workspace as viewed by the micro-robot system designers and operation designers. The micro-robot system designer is connected to the microgripper and micro-robot manufacturers. The micro-robot system designer can test their micro-robot system design integrated with microgripper and microrobot models. In this implementation and demonstration, the robotic system is assembled through the use of predefined fixed types of modules (joint modules and link modules) in distributed module inventories (repositories). These modules are published and can be accessed. The users or operation team can share their workspaces with the design team. The design team creates modules in the upper left corner while the robot system operation team makes the rest design. In this case the design team owns the session and the operation team join as a builder. Although builders cannot modify the modules created by other builders or owners, they can add new modules and utilize all services. For example, the operation team can use a service from a design module to obtain the robot accuracy and the open distance of the gripper and can build new modules in the workspace that utilize this information.

Similarly, the design team can also use services from the models published by the robot and gripper manufacturing team. Utilizing models provided by other designers is referred to as subscribing to a model. It is the responsibility of the design team to provide these data or to locate other models that can provide these data as services. The robot system managers want to evaluate the design from in term of costs and they may link their models 
to the design module to obtain the information services needed by their models. The design team has only published cost related aspects of their models. This means that the robot system managers can only observe elements of the design models that were published, as the designers wanted to protect their proprietary models.

The microgripper analysis and simulation and the microgripper design are also tightly coupled so that the designers from different design and simulation groups may also need to share a common model and access into the same workspace, although these groups may be in remote locations. The micro-assembly system is operated by means of a virtual microrobot manipulation system in which 3D models of the micro-components are manipulated virtually in a computer graphics constructed by VRML in the web scheme. The microassembly system simulator developed by the simulation team provides a new design tool of 3D MEMS by combining the possibility of the flexible assembly and the intuitive operations. Designers in the design team can use this tool to carry out the intuitive operations and simulations. This can help the designers to verify the design. When a simulating assembly or operating sequence is running, users can control microgripper open-close states, microrobot positions and orientations, micro-components positions and orientations by clicking on them. The user interface graphically displays micro-robot configurations, microgripper states, and the component states. The simulation results also help the designers in the design team to modify/redesign the design if necessary.

\subsection{Collaborative Microgripper Design and Analysis}

As discussed above, the microgripper is one of the important components in the micro robotic assembly system. In the collaborative design of mircogripper, many considerations and constraints should be emphasized and negotiated among designers on how to make sure the sensor could work well and the signals could be transferred back without any problem. Figure 14 depicts a mask layout, 2D \& 3D model and elastic simulation model for the gripper. Figure 14a shows the shape design of the microgripper. It is a symmetric structure consisting of six beams and four compliant linkages. In operation, the electricity current is applied to the beams of the thermal extension element. The beam heats up and lengthens, causing an angular deformation in the compliant mechanism and then open the gripper tips. Normally, the gripper is in its close position. Adding and switching off the voltage on the thermal extension element can open/close the gripper and thus an object can be gripped. Details can be found in the literature $[9,55]$.

Since the whole structure is symmetric, only half of it needs to be analyzed. The open distance of the microgripper mostly depends on the compliant mechanism part. Several parameters, including the widths and lengths of the linkages and the distance between the two linkages, influence the final function of the mechanism. The relationship between these parameters and the open distance is studied (see Appendix A). The algebraic equations for the description of the parameters, the relationships, and the conditions under which they apply facilitate dealing with the process of parametric design of microgripper in a systematic and efficient manner. Here, design diagram, a graph representation [23], is used, which is more effective for depicting the parameters and parameter relationships involved in the design. Furthermore, design diagrams bridge the representational gap that usually exists and provides a standard procedure. Constraint nets and data-flow graphs are closely related representation methods. 


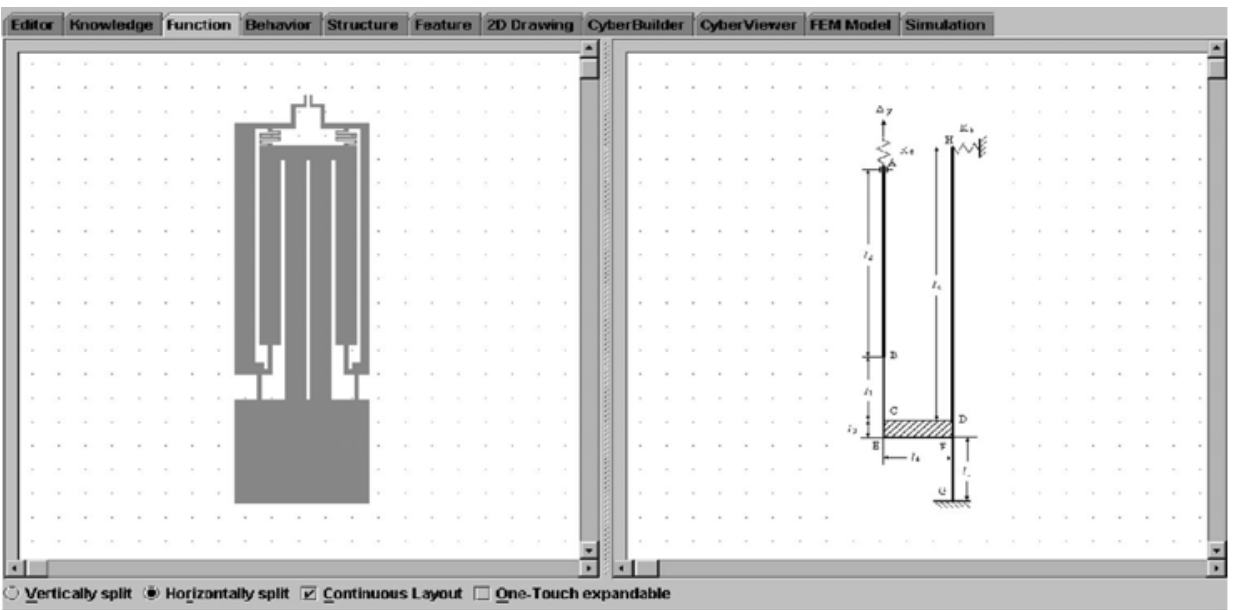

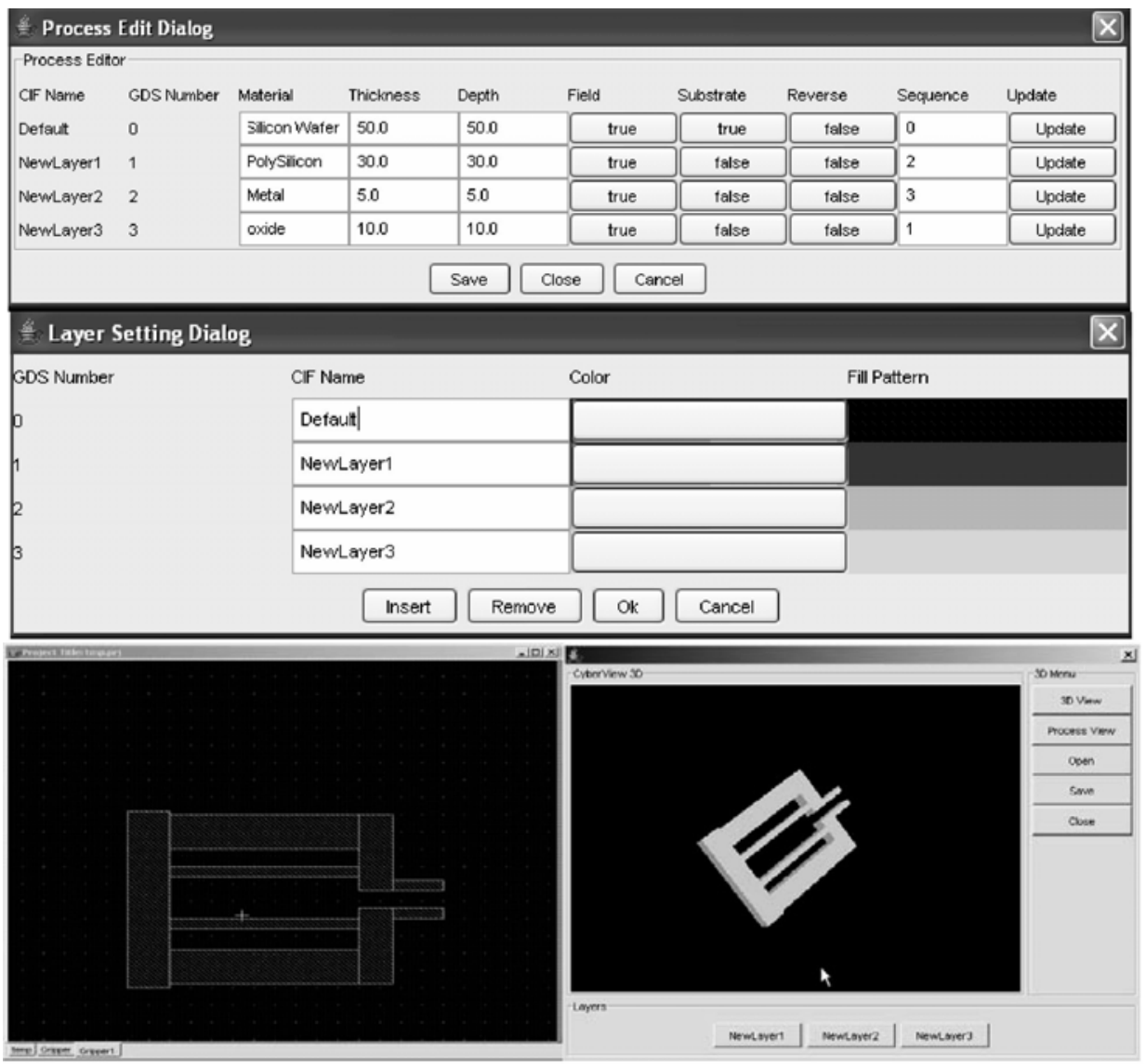

FIGURE 14. Microgripper design and simulation: mask layout, 3D model and elastic simulation model, (a) Layout and elastic simulation model, (b) Mask process and the generated 3D model (Java3D) (simplified demo) 


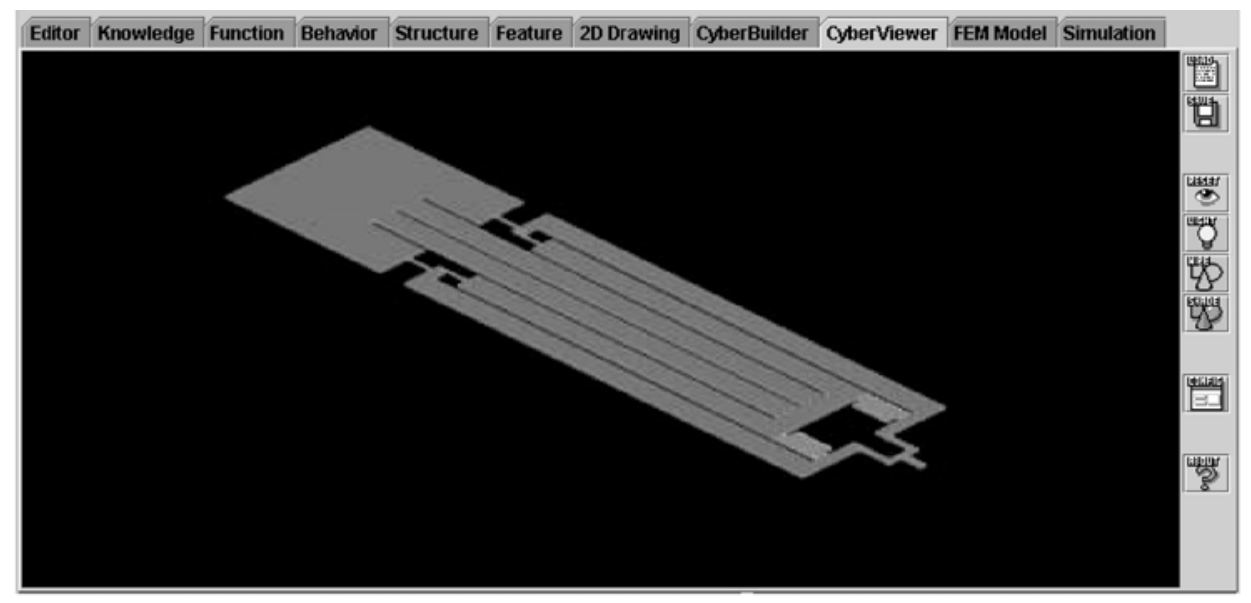

FIGURE 14c. Cyberview of the generated 3D model (VRML)

In the distributed module modeling and design schema, design modules are allowed to make decisions independently on certain parameters identified to be in the control of individual modules; these are decision parameters. Also, values of some exclusive and shared parameters are specified in the design requirements identified as given parameters. The strategy of concurrence can, however, result in conflict in shared parameters (i.e. conflict parameters), due to propagation of given parameters and decision parameters through engineering relationships between the parameters. Resolution of the conflict in values of the shared parameters requires some form of negotiation between the design modules to agree on mutually acceptable values. The classical models of utility and economic negotiation are useful to form the basis for a model of design negotiation. An illustrative situation is shown in Figure 15 in the form of a design diagram, where three modules $\mathrm{D}_{\mathrm{Mo}}, \mathrm{A}_{\mathrm{Mo}}$ and $\mathrm{E}_{\mathrm{Mod}}$ are the design module, analysis module and evaluation module respectively. Figure 16 shows the mesh generation and 2D \&3D FEM analysis GUI of the Web-MEMS Designer @ Analysis for the microgripper.

\subsection{Decision Support in Collaborative Design}

The consultation or decision support session in Web-MEMS Designer for collaborative design was implemented through the application of advisory systems for design support, for example, in assembly method selection, material and process selection, and so on. To illustrate the use of the manufacturing service module in Web-MEMS Designer for material and process selection, an example of a prototype gripper is explored to show the possibilities for making a full production run. Thus, it is necessary to use the process search, material search, and results survey mode [73]. The specifications are made for the procedures of process search and material search. The process search is for the lowest possible cost over a long production cycle. At the end of the process search, Electro-Dischargeable Machine (EDM) (rank 1.00) was ahead of the only other possibility, Etch (rank 0.96). Similarly, after material search, the system generated six viable materials, with carbon steel ranking the highest at 1.00 and aluminum \& alloys ranking at 0.98 . Furthermore, after process search 


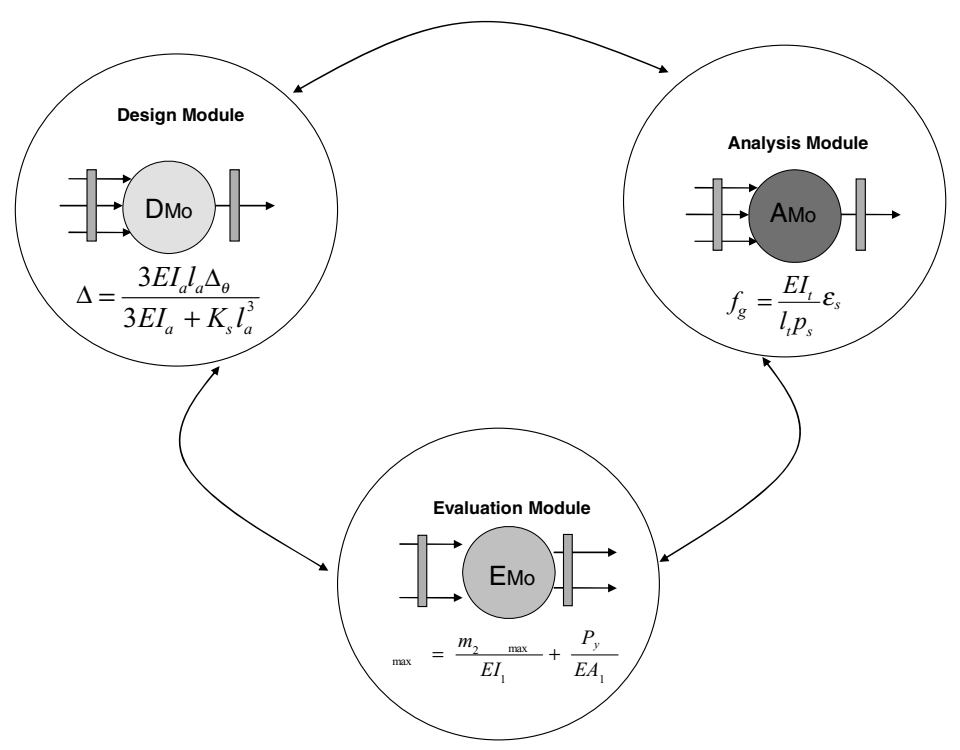

FIGURE 15. Microgripper design and analysis module network

and material search, the "Final Result" button would be enabled and clicked to combine the results of both searches to find the best material/process combination. As shown in Figure 17, the two boxes at the top is a summary of the viable materials and processes, and the final box lists all of the feasible combinations, taking into account a compatibility factor between each process and material. Thus, EDM with carbon steel is the best choice, with etched stainless steel second.

On completion of the reasoning process, Web-MEMS Designer returns the score obtained for this design with respect to this attribute and indicates in the result page the Pass/Fail status of each design parameter. To request an explanation of the evaluation, the user can click on the button of the "Explain the Result" on the results' page shown above. The explanation may consist of the rationale for the score in terms of justifications and references, both to the design literature and various on-line resources. It also shows an example of how on-line expert design knowledge and resources can be accessed during the design process.

\section{SUMMARY AND FUTURE WORK}

This chapter presented a web-based design platform for supporting collaborative MEMS design over the Internet and web. A two-tiered client (browser) / knowledge server architecture was adopted to allow experts and designers to publish and subscribe modeling services on the web. The proposed KS-DMME framework is built upon to provide module network architecture for integrating modeling services. In the module network, design resources, models, data, and activities are not centralized nor concentrated in one location. They are distributed among many companies, designers, or design participants working 

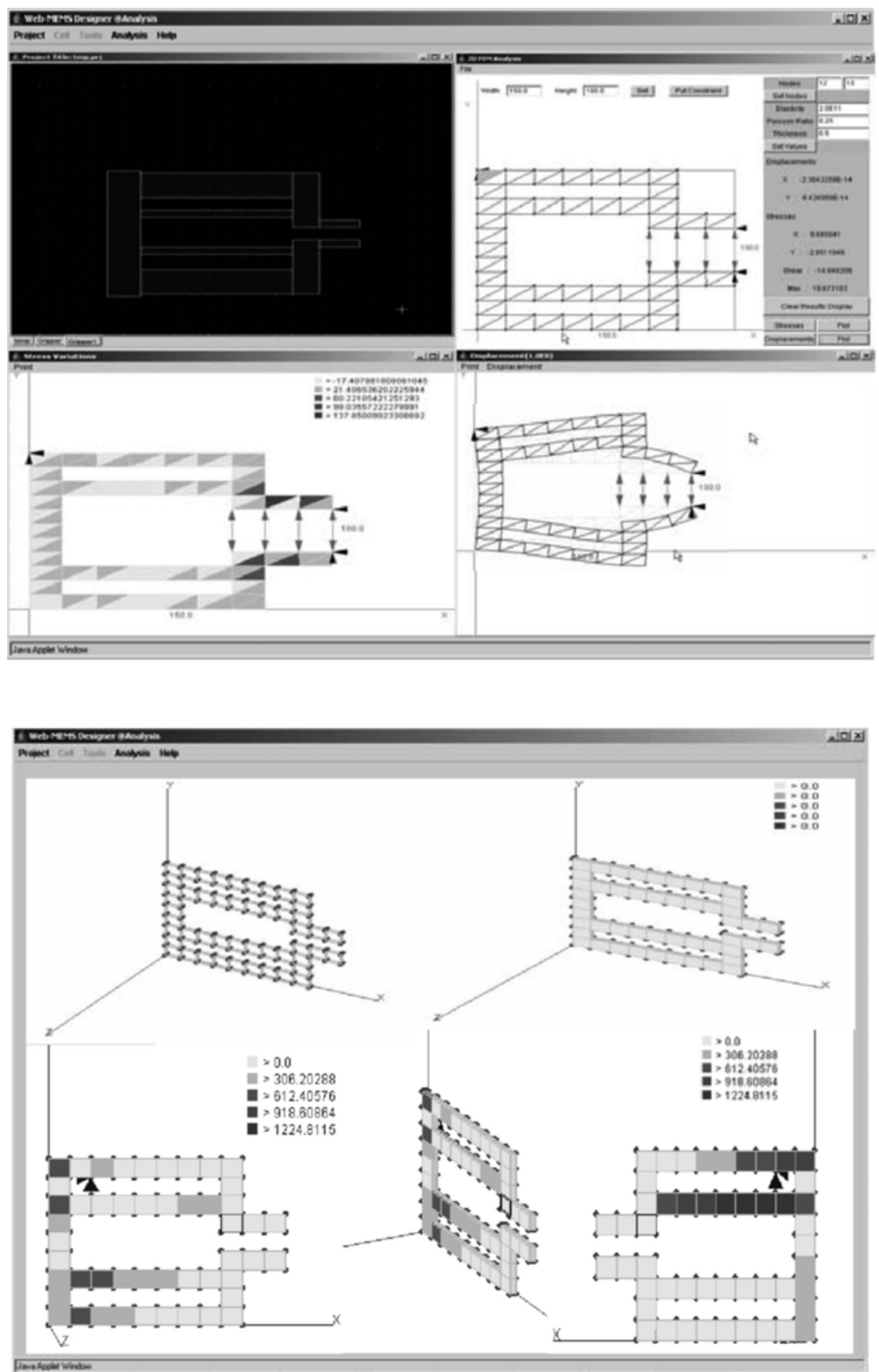

FIGURE 16. Microgripper 2D and 3D FEM analysis by Web-MEMS Designer @ Analysis (Demo), (a) 2D FEM analysis, (b) 3D FEM analysis 


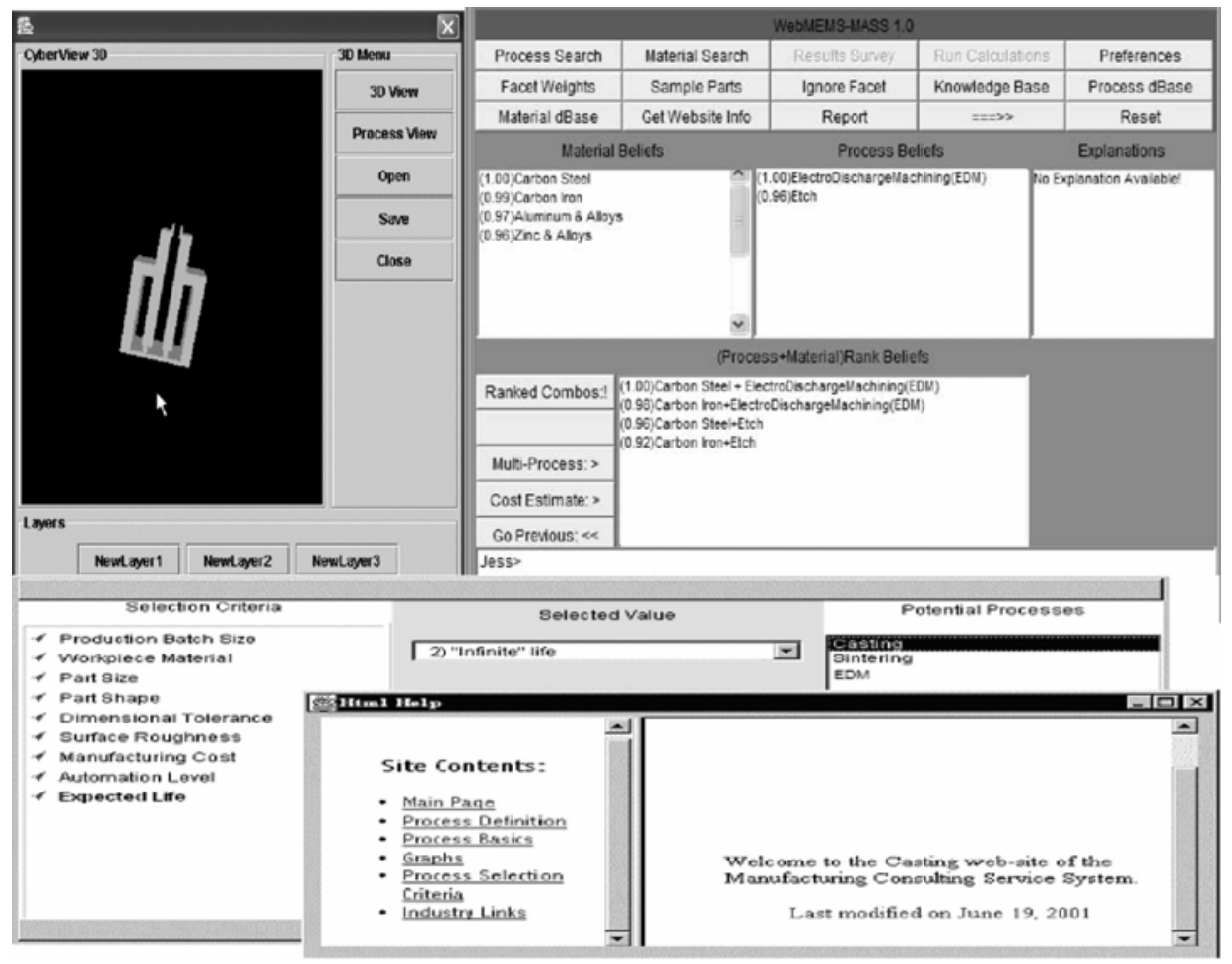

FIGURE 17. Process and material search in the design process and final results

together over the internet/intranet. When module services are connected, the resultant service exchange network creates a concurrent integrated system model or a module network that invokes a chain of service requests if needed to provide correct information. To provide distributed designers with a tool for collaboratively building the concurrent integrated design system models, the KS-DMME framework is extended to be a computer network environment focusing on the design and simulation for MEMS. MEMS design modules are created by fully implementing the locally defined modules and subscribing to the services of remote modules. The implementation of Web-MEMS Designer system hides the details of the remote interaction mechanism from the user but allows the MEMS designer to model interactions between local and remote modules in a transparent manner. In turn designers can selectively publish modeling services for use by others. The microgripper design for micro-robot assembly example illustrates the concept and different models of collaboration supported by the prototype implementation.

The knowledge supported design system can help companies capture and archive their design knowledge and manage the design process. It also supports communication and teamwork by sharing the most up-to-date design information. Designers, especially novices, can benefit from retrieval of knowledge about previous designs by abstracting information and applying it to a new design or by gaining insight into how an earlier related product was designed. By making use of the design knowledge, companies are expected to improve 
the design process for more innovative products and reducing product development cycle time. As a kernel of the knowledge supported design system, the design decision support system can help design teamsmake better decisions.

The preliminary implementation of the Web-MEMS Designer system illustrates the potential of KS-DMME framework for MEMS design and simulation. When fully implemented and integrated with other computer-based collaboration tools, the Web-MEMS Designer system will provide designers with a powerful infrastructure for collaborative MEMS design. However, there exists a large amount of work to be done both on the particular design paradigms or methodologies for MEMS and the system development. For example, the framework should accommodate top-down and bottom-up approaches or models in the context of both traditional sequential design processes and concurrent design for MEMS devices or systems. In a collaborative design environment, there are also a number of fundamental issues yet to be addressed such as knowledge base evolutionary maintenance, model interface standard, computational strategy for resolving circular dependencies in the DMME model, parallel service request invocation, etc. In addition, other aspects such as human interaction and knowledge sharing will still require the integration of additional support tools with the framework (e.g., ontology, etc.). The project described in this chapter is still in progress.

\section{DISCLAIMER}

The bulk of the work reported here by the author was conducted during his tenure at Nanyang Technological University, Singapore. No approval or endorsement of any commercial product, service or company by the National Institute of Standards and Technology is intended or implied.

\section{REFERENCES}

1. An, S. (2000), MEMS CAD, http://myhome.dreamx.net/piyo123/default.html (not accessible now)

2. Abdrzej, P., An Integrated Multidisciplinary CAD/CAE Environment for Micro-Electro-Mechanical System (MEMS), CFD Research Corp., AL, 1998.

3. Bay, Eric, Seah, Calvin, Zha, X.F., and Du, H., A Web-Enabled Drawing Applet for Masking Process in MEMS, Final Year Project Report, School of Mechanical and Production Engineering, Nanyang Technological University, Singapore, 2000.

4. Bic, L.F., Fukuda, M., and Dillencourt, M.B., Distributed Computing Using Autonomous Objects, IEEE Computer, 1995;55-61.

5. Bliznakov, P.I., Shah, J.J., Jeon, D.K., and Urban, S.D., Design Information System Infrastructure to Collaborative Design in a Large Organization, Proceedings of ASME DETC, Boston, MA, 1995, Vol. 1, pp. 1-8.

6. Bliznakov, P.I., Design Information Framework to Support Engineering Design Process, Dissertation, Arizona State University, AZ, 1996.

7. Cary, S.B. and Zhang, Y., CAEMEMS: An Integrated Computer Aided Engineering Work Bench for Micro-Electro-Mechanical Systems, Proceedings of 1990 IEEE Micro-Electro-Mechanical Systems Workshop, 1990.

8. Case, M.P. and Lu, S.C.-Y., Discourse Model for Collaborative Design, Computer-Aided Design, 1996;28(5):333-345.

9. Du, H., Su, C., Lim, M.K., and Jin, W.L., A Micro-machined Thermally-driven Gripper: a Numerical and Experimental Study, Smart Material and Structure, 1999;8:616-622. 
10. Eriksson, H., Expert systems as Knowledge servers. IEEE Expert, 1996;14(3):14-19.

11. Frost, H.R. and Cutkosky, M.R., Design for Manufacturability via Agent Interaction, Proceedings of 1996 ASME DETC, Irvine, California, 1996.

12. Gad-el-Hak, M., The MEMS Handbook, CRC Press, 2002.

13. Gilbert, J.R., Osterberg, P.M., Harris, R.M., Ouma, D.O., Cai, X., Pfajfer, A., White, J., and Senturia, S.D., Implementation of A MEMCAD System for Electrostatic and Mechanical Analysis of Complex Structures from Mask Descriptions, Proceedings of MEMS'93, Fort Lauderdale, FL, 1993, pp. 207-212.

14. Gilbert, J.R., Integrating CAD Tools for MEMS Design, Computer, 1998, pp. 99-101.

15. Giridharam, M.G., Stout., P., Yang, H.Q. et. al., Multi-Disciplinary CAD System for MEMS, Journal of Modeling and Simulation of Microsystems, 2001;2(1):43-50.

16. Graham, I., Object-Oriented Methods, Addison-Wesley, 1994.

17. Hardwick M. and Spooner, D., An Information Infrastructure for a Virtual Manufacturing Enterprise, Proceedings of Concurrent Engineering: A Global Perspective, McLean, VA, 1995, pp. 417-429.

18. Hahn, K. and Bruck, R., Web-based Design Tools for MEMS-Process Configuration, Technical Proceedings of the 1999 International Conference on Modeling and Simulation of Microsystems, 1999, pp. 346349.

19. Schneider, C., Schumer, C., and Brück, R., An Internet-Based Design Tool in 3-Tier Component-Architecture for Adaptive MEMS-Design, The Eleventh Micromechanics Europe Workshop (MME '00) (Uppsala, 2000), Uppsala University: Eigenverlag, 2000.

20. IONA, Orbix2 Programming Guide, IONA Technologies Ltd., 1997.

21. Karam, J.M., Bcourtois, B., and Boutamine, H. et al., CAD and Foundries for Microsystems, DAC97, Anaheim, California, 1997.

22. Judy, J.W., Microelectromechanical Systems (MEMS): Fabrication, Design and Applications, Smart Materials and Structures, 2001;10:1115-1134.

23. Kannapan, S.M. and Marshek, K.M., An Approach to Parametric Machine Design and Negotiation in Concurrent Engineering, in Kusiak, A., Chapter 20, Concurrent Engineering: Automation, Tools, and Techniques, 1993, John Wiley \& Sons, Inc., pp. 509-533.

24. Koppleman, G.M., OYSTER, A Three Dimensional Structural Simulator for Micro-Electro-Mechanical Design, Sensors and Actuators, 1989, Vol. 20.

25. Korvink, J.G., Funk, J., Roos, M., Wachutka, G., and Baltes, H., SESES: A Comprehensive MEMS Modeling System, IEEE International Workshop on Micro Electro Mechanical Systems, MEMS'94, Osio, Japan, 1994, pp. 22-27.

26. Lewis J.W. and Singh, K.J., Electronic Design Notebooks (EDN): Technical Issues, Proceedings of Concurrent Engineering: A Global Perspective, McLean, VA, 1995, pp. 431-436.

27. Lin, H.C., JAVAMESH-A Two Dimensional Triangular Mesh Generator for Finite Elements, Master Thesis, University of Pittsburgh, PA, 1997.

28. Li, Y.L., Shao, X.Y., Li, P.G., and Liu, Q., Design and Implementation of A Process-oriented Intelligent Collaborative Product Design System, Computer in Industry, 2004;53:205-229.

29. MADEFast (1999), http:// madefast.stanford.edu/

30. Maseeh, F., A CAD Architecture for Microelectromechanical Systems, Proceedings of IEEE Conference on Micro Electro Mechanical Systems (MEMS'90), Napa Valley, CA, 1990, pp. 44-49.

31. Maseeh, F., A Novel Multidimensional Semiconductor Material Analysis Tool, Solid State Technology, 1994;37:83-84.

32. Madou, M., Fundamentals of Microfabrication, CRC Press, 1997.

33. Owen, J., STEP - An Introduction, Winchester, 1993.

34. Osterberg, P.M. and Senturia, S.D., MEMBUILDER: An Automated 3D Solid Model Construction Program for Micro-Electro-Mechanical Structures, Proceedings of TRANSDUCERS'95, Stockholm, Sweden, 1995, pp. 21-24.

35. Pahng, F., Senin, N., and Wallace, D., Modeling an Evaluation of Product Design Problems in a Distributed Design Environment, CD ROM Proceedings of ASME DETC, Sacramento, CA, 1997.

36. Pahng, F., Bae, S.H., and Wallace, D., Web-based Collaborative Design Modeling and Decision Support, Proceedings of DETC'98, Atlanta, Georgia, USA, 1998.

37. Pena-Mora, F., Sriram, R., and Logcher, R., SHARED DRIMS: SHARED Design Recommendation-Intent Management System, Enabling Technologies: Infrastructure for Collaborative Enterprises, IEEE Press, 1993, pp. 213-221. 
38. Pena-Mora, F., Sriram, R., and Logcher, R., Conflict Mitigation System for Collaborative Engineering, AI EDAM-Special Issue of Concurrent Engineering, 1995;9(2):101-123.

39. Perterson, K.E., Dynamics Micromechanics on Silicon: Techniques and Devices, IEEE Trans. Electron Devices ED-25, 1978;1241-1250.

40. Petrie, C., Cutkosky, M., and Park, H., Design Space Navigation as a Collaborative Aid, Proceedings of Third International Conference on Artificial Intelligence in Design, Lausanne, Switzerland, 1994.

41. Rao, D.J., The Finite Element Method Site, http://members.tripod.com/dattaraj_rao/FEM/index.html

42. Salzberg S. and Watkin, M., Managing Information for Concurrent Engineering: Challenges and Barriers, Research in Engineering Design, 1990;(2):35-52.

43. Saha, D. and Chandrakasan, A.P., A Framework for Distributed Web-based Microsystem Design, Proceedings Sixth IEEE workshops on Enabling Technologies: Infrastructure for Collaborative Enterprises, Cambridge, MA, 1997, pp. 69-74.

44. Schneider, P., Huck, E., Reitz, S., Parodat, S., Schneider, A., and Schwarz, P., A Modular Approach for Simulation-based Optimization of MEMS, Design, Modeling and Simulation in Microelectronics, SPIE Proceedings Series, Singapore, 2000, 4228.

45. Senturia, S.D. and Howe, R.T., Mechanical Properties and CAD, Lecture Notes, MIT, Boston, MA, 1990.

46. Senturia, S.D. and Harris, R.M., A Computer-Aided Design System for Micro-Electro-Mechanical Systems (MEMCAD), Journal of Micro-Electro-Mechanical Systems, 1992;1(1).

47. Senturia, S.D., CAD Challenges for Microsensors, Microactuators, and Microsystems, Proceedings of the IEEE, 1998;86(8):1611-1626.

48. Siegel, J., CORBA: Fundamentals and Programming: OMG, 1996.

49. Sinha, N., Modeling for Effective Computer Support to MEMS Product Development, Master Thesis, University of Saskatchewan, Canada, 2004.

50. Singh, A.K., CONSENS-An IT Solution for Concurrent Engineering, Proceedings of Concurrent Engineering: A Global Perspective, McLean, VA, 1995, pp. 635-644.

51. Sobolewski M.W. and Erkes, J., CAMnet Architecture and Applications, Proceedings of Concurrent Engineering: A Global Perspective, McLean, VA, 1995, pp. 627-634.

52. Sriram D. and Logcher, R., The MITDICE Project, IEEE Computer, 1993, pp. 64-65.

53. Sriram, R.D., Distributed and Integrated Collaborative Engineering Design, Sarven Publishers, Glenwood, MD 21738, USA, 2002.

54. Stodes, M., Managing Engineering Knowledge: MOKA Methodology for Knowledge Based Engineering Applications, MOKA Consortium, London.

55. Su, C., Development of Three MEMS Devices: A Microgripper, A Micromechanism and A Microaccelerometer, M. Eng Theses, Nanyang Technological University, Singapore, 1999.

56. Tadigadapa, S.A. and Najafi, N, Developments in Microelectromechanical Systems (MEMS): A Manufacturing Perspective, Transaction of the ASME, Journal of Manufacturing Science and Engineering, 2003;125:816823.

57. Tay, Francis E.H., Collaborative Design Software for MEMS, Proceedings of SPIE- The International Society for Optical Engineering, Proceedings of the 1999 Education in Microelectronics and MEMS, Oct 28-Oct 29, 1999, Vol. 3894, pp. 152-158.

58. Toh, W.Y., Development of a Web Database System for Manufacturing Processes of MEMS, Final Year Project Report, School of Mechanical and Production Engineering, Nanyang Technological University, Singapore, 2001.

59. Toye, G. Cutkosky, M.R., Tenenbaum, J.M., and Glicksman, J., SHARE: A Methodology and Environment for Collaborative Product Development, Proceedings of Second Workshop on Enabling Technologies: Infrastructure for Collaborative Enterprises, Morgantown, West Virginia, 1993, pp. 33-47.

60. Westerberg, A.W., Coyne, R. Cuningham, D. Dutoit, A. Gardner, E. Konda, Levy, S. Monarch S.I., Patrick, R. Reich, Y. Subrahmanian, E., Terk, M., and Thomas, M., Distributed and Collaborative Computer- Aided Environment in Process Engineering Design, Proceedings of ISPE, 1995.

61. Wilson, N.M., Liang, S. Pinsky, P.M., and Dutton, R.W., A Novel Method to Utilize Existing TCAD Tools to Build Accurate Geometry Required for MEMS simulation, Proceedings of Modeling and Simulation of Microsystems, April 19-21, 1999, pp. 120-123.

62. Wilson, N.M., Wang, K. Yergeau, D., and Dutton, R.W., GEODESIC: A New and Extensible Geometry Tool and Framework with Application to MEMS, Proceedings of Modeling and Simulation of Microsystems, March 27-29, 2000, pp. 716-719. 
63. Wilson, N.M., Wang, K. Yergeau, D., and Dutton, R.W., Internet-based Prototyping of Micro-ElectroMechanical Systems, ICCES 2000, 2000.

64. Wood III, W.H. and Agogino, A.M., Case-based Conceptual Design Information Server for Concurrent Engineering, Computer-Aided Design, 1996;8(5):361-369.

65. Yoshimura, S., Kowalczyk, T., Wada, Y., and Yagawa, G. (1998), A CAE System for Multidisciplinary Design and Its Interface in Internet, Transactions of JSCES, http://save.k.u-tokyo.ac.jp/jsces/trans/trans1998/ n19980004/n19980004.html.

66. Zha, X.F. and Du, H., Knowledge Intensive Methodology for Design and Simulation of MicroEelectro-Mechanical System (MEMS) Devices, Report-MEMS CAD/CAE/CE for Singapore NSTB Project ARC 5/97, School of Mechanical and Production Engineering, Nanyang Technological University, Singapore, 1999.

67. Zha, X.F. and Du, H., Web-Based Knowledge Intensive Collaborative Design Modeling and Decision Support for MEMS, Proceedings of International Conference on Engineering and Technological Sciences, Beijing, China, 2000.

68. Zha, X.F. and Du, H., A World Wide Web Based Manufacturing Advisory Service System for Fabrication/Packaging Process/Material Selection in Concurrent Collaborative Design of MEMS Devices, Proceedings of International Conference on Material for Advanced Technologies, Singapore, 2001a.

69. Zha, X.F. and Du, H., Web-Based Knowledge Intensive Collaborative Design Framework for MEMS, Proceedings of International Workshop on MEMS, Singapore, $2001 \mathrm{~b}$.

70. Zha, X.F. and Du, H., Mechanical Systems and Assemblies Modeling Using Knowledge Intensive Petri Net Formalisms, Artificial Intelligence for Engineering Design, Analysis, and Manufacturing (An international Journal), 2001c;15(3):145-171.

71. Zha, X.F. and Du, H., An Integrated Representational Model for Concurrent Assembly Design and Planning, Proceedings of 4th IEEE International Symposium on Assembly and Task Planning, Fukuoka, Japan, 2001d.

72. Zha, X.F. and Du, H., Web-based Knowledge-Intensive Support Framework for Collaborative Design of MEMS, Journal of Microengineering and Micromechanics, 2002;12:509-522.

73. Zha, X.F. and Du, H., Manufacturing Process and Material Selection in Concurrent Collaborative Design of MEMS Devices, Journal of Microengineering and Micromechanics, 2003;13:512-524.

\section{APPENDIX A: ANALYSIS OF MICROGRIPPER}

The analysis of the microgripper is based on the work $[9,55]$. In the model shown in Figure 12a, the expansion element is replaced by a spring with stiffness $K_{\mathrm{e}}$, and $K_{\mathrm{e}}=$ $\mathrm{EA}_{\mathrm{e}} / l_{\mathrm{e}}$, here $\mathrm{E}$ is the Young's modulus and $\mathrm{A}_{\mathrm{e}}$ and $l_{\mathrm{e}}$ are cross-section area and length of the thermal extension element. The transfer element and the two compliant linkages are treated as Euler beam. Because the two linkages have the same functions, they should have the same dimensions. As the figure shows, one linkage is fixed on the basement and a small displacement in $\mathrm{Y}$ direction is inputted at the end of the other one. A much shorter and wider beam connects the two compliant linkages, and thus the beam can be considered as a rigid body. The gripper arm is also treated as an Euler beam and another spring with stiffness coefficient $\mathrm{K}_{\mathrm{s}}$ is used to simulate the supporting spring structure.

Figure A1 shows force condition of the connector beam. From the force equilibrium principle, it can be known that the forces added on point $\mathrm{C}$ and $\mathrm{F}$ should have the same values and opposite directions. $\mathrm{m}_{1}$ and $\mathrm{m}_{2}$ are the moments added on the connector beam by the linkages. The sum of the moments on the beam should be zero, therefore,

$$
m_{1}+m_{2}+l_{3} P_{x}-l_{4} P_{y}+K \Delta_{\theta}=0
$$




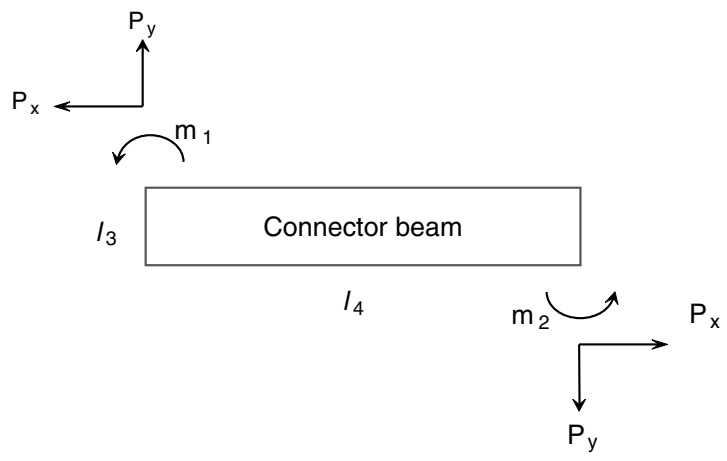

FIGURE A1. Force illustration of the connector beam

where, $\Delta_{\theta}$ is the rotation angle of the connector beam and $\mathrm{K}$ represents the equivalent rotation stiffness of the gripper arm at point $\mathrm{D}$ and should be

$$
K=\frac{3 E I_{a} K_{s} l_{a}^{2}}{3 E I_{a}+K_{s} l_{a}^{3}}
$$

where, $I_{\mathrm{a}}$ and $l_{\mathrm{a}}$ denote the second moment of area and length of the arm. As the rotation angle $\Delta_{\theta}$ is normally only a small value [55], $\sin \Delta_{\theta}$ can be considered the same as $\Delta_{\theta}$, $\sin \Delta_{\theta} \approx \Delta_{\theta}$. In this case, the difference between the $x$-coordinates of point $\mathrm{C}$ and $\mathrm{F}$ reduces $l_{3} \Delta_{\theta}$ and the difference between the $y$-coordinates of point $\mathrm{C}$ and $\mathrm{F}$ increases $l_{4} \Delta_{\theta}$. Thus,

$$
\begin{aligned}
& -\frac{1}{2}\left[\frac{1}{E I_{1}} l_{1}^{2}+\frac{1}{E I_{2}} l_{2}\left(2 l_{1}+l_{2}\right)\right] m_{1}-\frac{1}{2} \frac{1}{E I_{1}} l_{1}^{2} m_{2} \\
& +\frac{1}{3}\left[\frac{2}{E I_{1}} l_{1}^{3}+\frac{1}{E I_{2}}\left(l_{2}^{3}+3 l_{2}^{2} l_{1}+3 l_{2} l_{1}^{2}\right)\right] P_{x}-l_{3} \Delta_{\theta}=0
\end{aligned}
$$

and,

$$
\left(2 \frac{1}{E A_{1}} l_{1}+\frac{1}{E A_{2}} l_{2}+\frac{1}{K_{e}}\right) P_{y}+l_{4} \Delta_{\theta}=\Delta_{y}
$$

where, $I_{1}, l_{1}$ and $I_{2}, l_{2}$ represent the second moment of area and length of the linkages and the displacement transfer element respectively. The ends of beam BC and beam GF should also rotate $\Delta_{\theta}$ and they can be considered as cantilever beam, then $\mathrm{m}_{1}, \mathrm{~m}_{2}, \mathrm{P}_{x}$ and $\Delta_{\theta}$ should have the following relationship,

$$
\left(\frac{1}{E I_{1}} l_{1}+\frac{1}{E I_{2}} l_{2}\right) m_{1}-\frac{1}{2}\left[\frac{1}{E I_{1}} l_{1}^{2}+\frac{1}{E I_{2}} l_{2}\left(2 l_{1}+l_{2}\right)\right] P_{x}-\Delta_{\theta}=0
$$

and

$$
\frac{1}{E I_{1}} l_{1} m_{2}-\frac{1}{2} \frac{1}{E I_{1}} l_{1}^{2} P_{x}-\Delta_{\theta}=0
$$


This means the rotation angle $\Delta_{\theta}$ is generated by the combination of the moment and the force.

In total, there are five variables, namely $\mathrm{m}_{1}, \mathrm{~m}_{2}, \mathrm{P}_{\mathrm{x}}, \mathrm{P}_{\mathrm{y}}$, and $\Delta_{\theta}$ and we have five equations (A1)-(A6). Hence, if the geometric dimensions of the microgripper are determined, these variables can be found, and then the open distance can be calculated out as

$$
\Delta=\frac{3 E I_{a} l_{a} \Delta_{\theta}}{3 E I_{a}+K_{s} l_{a}^{3}}
$$

Further, to evaluate the strength of gripper, the location and the value of the maxim strain need to be determined. In this design, deforming some certain parts transfers the displacement, and the strain is concentrated mainly in the two compliant linkages. The two linkages have the same rotation angles at their ends, and the rotation stiffness of the beam FG is stronger than the stiffness of the combination of beam $\mathrm{BC}$ and beam $\mathrm{AB}$. Therefore, $m_{2}$ should be bigger than $m_{1}$ and the maximum strain occurs at the left side of the beam FG as follows:

$$
\varepsilon_{\max }=\frac{m_{2} \rho_{\max }}{E I_{1}}+\frac{P_{y}}{E A_{1}}
$$

where, $\rho_{\max }$ should be half of the width of the beam FG. The gripping force can be expressed as

$$
f_{g}=\frac{E I_{t}}{l_{t} \rho_{s}} \varepsilon_{s}
$$

where $\varepsilon_{s}$ denotes strain of the piezoresistive film, $\mathrm{I}_{\mathrm{t}}$ and $l_{t}$ denote the second moment of area and length of the tip and $\rho_{\mathrm{s}}$ denotes how far the piezoresistive film is away from the central line of the tip.

\section{APPENDIX B: GENERIC P/T NET FOR MICRO ASSEMBLY REPRESENTATION AND CONCEPTUAL MEMS DESIGN}

A generic graph can be described as a two tuple $G=G(V, E)$, where $V$ is the set of nodes and $\mathrm{E}$ is the set of connecting arcs which link between nodes. If each arc in a graph has a direction, then the graph is a directed graph. If a node is categorized into two classes: place $(\mathrm{P})$ node and transition $(\mathrm{T})$ node, then a place-transition $(\mathrm{P} / \mathrm{T})$ net graph model, as shown in Figure B1 (a), can be formally defined as: PTN $=\{\mathrm{P}, \mathrm{T}, \mathrm{A}, \mathrm{W}\}$, where, $\mathrm{P}=\left(\mathrm{p}_{1}\right.$, $\left.\mathrm{p}_{2}, \ldots, \mathrm{p}_{\mathrm{m}}\right)$ is the place node set; $\mathrm{T}=\left(\mathrm{t}_{1}, \mathrm{t}_{2}, \ldots, \mathrm{t}_{\mathrm{n}}\right)$ is the transition node set; and $\mathrm{A}$ is a arcs set which links between place node and transition node, and has the characteristics of $: \mathrm{P} \cap \mathrm{T}=\phi, \mathrm{P} \cup \mathrm{T} \neq \phi$, and $\mathrm{A} \subseteq(\mathrm{P} \times \mathrm{T}) \cup(\mathrm{T} \times \mathrm{P})$; and $\mathrm{W}: \mathrm{A} \rightarrow\{0,1\}$ is an association weight function on arcs, $\forall \mathrm{a} \in \mathrm{A}, W(\mathrm{a})=w_{\mathrm{i}}, w_{\mathrm{i}}$ is the weight of arc a. Correspondingly, if each arc in the $\mathrm{P} / \mathrm{T}$ net graph has a direction, then it becomes a directed $\mathrm{P} / \mathrm{T}$ net graph, as shown in Figure B1(b).

Based on the definition, a Petri net consists of places (P) and transitions (T), which are linked to each other by arcs. Therefore, a Petri net graph is in fact a directed P/T net graph. If the net activities are based on a vision of tokens moving around an abstract network, in which 


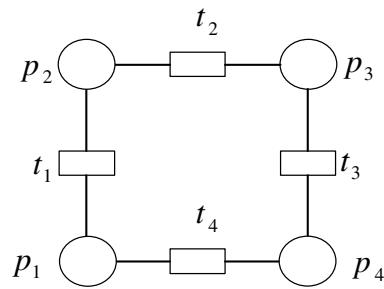

(a) $\mathrm{P} / \mathrm{T}$ net

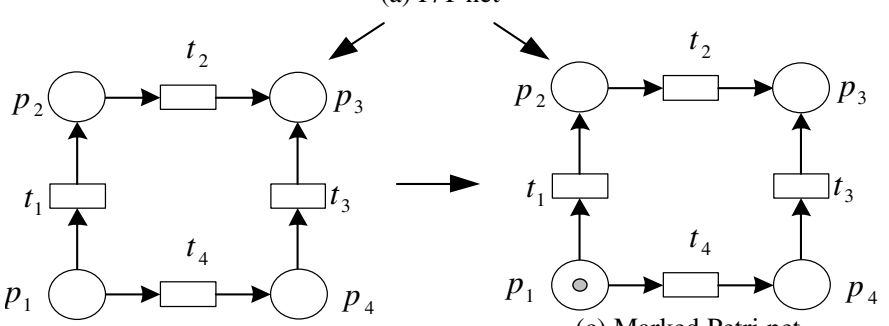

(b) Directed Petri net

(c) Marked Petri net

FIGURE B1. Generic P/T net graph

tokens are conceptual entities that model the objects and appear as small solid dots moving in a real network, a marked Petri net, as shown in Figure B1(c), can be formally defined as a 5-tuple, $\mathrm{PN}=\left(\mathrm{PTN}, \mathrm{M}_{0}\right)=\left(\mathrm{P}, \mathrm{T}, \mathrm{A}, \mathrm{W}, \mathrm{M}_{0}\right)$, where, $\mathrm{PTN}$ is a directed $\mathrm{P} / \mathrm{T}$ net; $\mathrm{P}, \mathrm{T}, \mathrm{W}, \mathrm{A}$ are the same as above definitions; $\mathrm{M}_{0}: \mathrm{P} \rightarrow\{0,1,2, \ldots\}$ is the initial marking. The Petri net graph is a graphic representation of Petri net structure and visualizes the reasoning rules.

By the generic P/T net scheme, we mean that a P/T net model of a problem is first described as a kind of "template", and the models of the particular sub-problems are then established as instances of the template. Since incorporating a P/T net model into a general problem description scheme in AI generates the proposed generic P/T net, existing AIbased problem solving strategies such as search, reasoning and (fuzzy) expert systems are applicable for the generic P/T net modeling and analysis. Thus, the generic P/T net is knowledge intensive, also called knowledge P/T net. For more descriptions, please refer to $[70,71]$.

As an assembly is composed of parts or components and connectors (joints), and a single part is composed of physical features, the different levels of assembly actually form a hierarchy, which utilizes the relationships between different parts of assembly and even different features of part. The "place-transition" $(\mathrm{P} / \mathrm{T})$ model is used to represent the mechanical systems and assemblies, in which each part is represented as a place and each connector (joint) is represented as a transition. Therefore, a mechanical system or assembly can be viewed as a hierarchical P/T net, called Assembly_Model, and accordingly a subsystem or subassembly is a sub P/T net. Using modular representation, a sub P/T net (object) can be described as either a macro place or transition. This is mainly dependent on its function as either a component or a joint or a connector. Token data abstraction and dynamic distribution can be used for knowledge representation in describing the structure and system state changes. In addition, the generic $\mathrm{P} / \mathrm{T}$ net can be used for intelligent modeling of assembly functions and behaviors, and design, planning and simulation of assembly processes and assembly systems. 


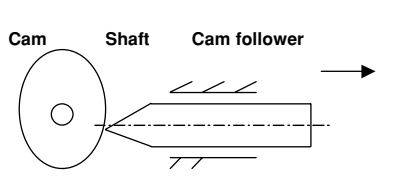

(a) Cam mechanism
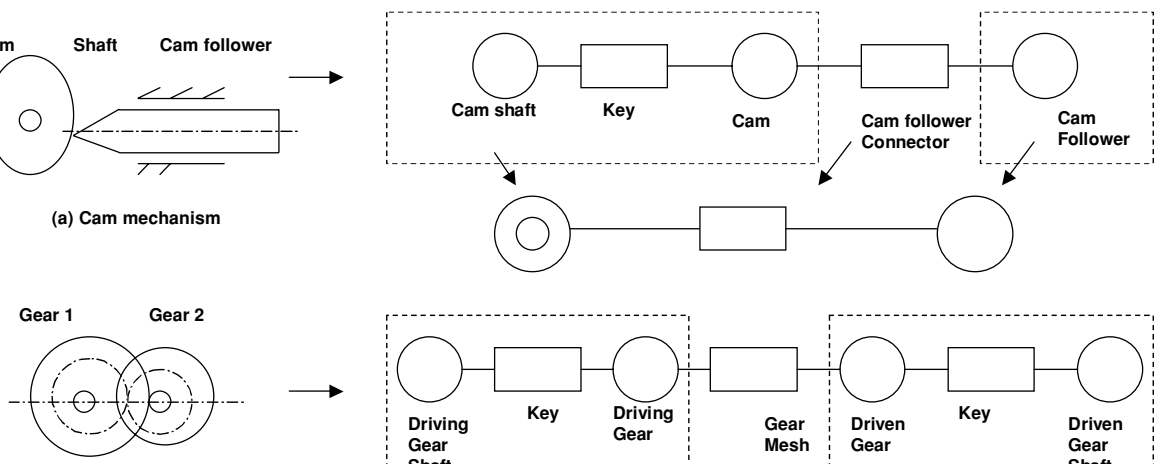

(b) Gear mechanism
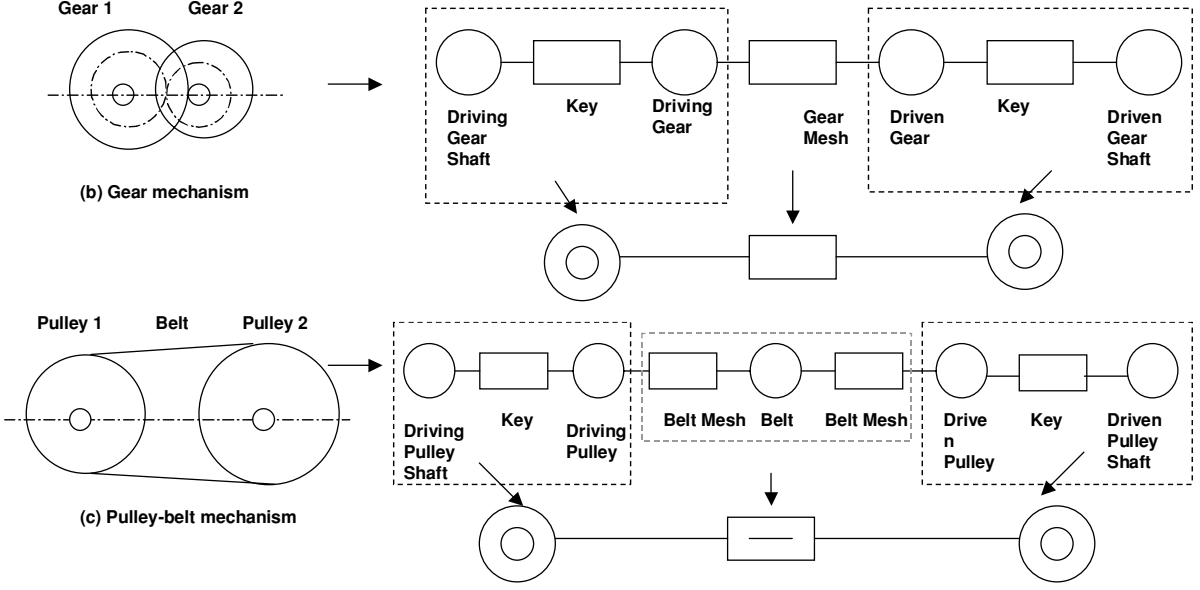

FIGURE B2. P/T net-based representation for micro primitive mechanisms

A multi-level P/T net could be generated by network modeling from top to bottom. Any conceptual design product can be considered as a combination of places and transitions. Transitions make places work normally by connecting them. Each primitive mechanism can be viewed as a micro place node or micro place node in the P/T net-based hierarchical structure of the mechanical product. Each primitive mechanism can also be represented by a P/T net. Figure B2a, b and c show a P/T net-based representation of three primitive mechanisms (Cam, gear mesh, and pulley-belt), respectively.

$\mathrm{P} / \mathrm{T}$ net model could also allow for the possibility that the properties take some values in the form of a fuzzy set over a base range. For example, a transition with a motion transmission function might become a gear pair; a transition with a fixing function might be a collection of geometric mating surfaces such as a cylinder and shoulder. Since places and transitions for components and connectors are conceptually fuzzy, they might form a fuzzy $\mathrm{P} / \mathrm{T}$ net to represent a sub-assembly during later stages of design.

\section{APPENDIX C: MEMS MODELING AND SIMULATION RELATED WEBSITES}

3-D Modeling and Simulation

ANSYS (http://www.ansys.com/products/multiphysics.shtml)

CFD Research Corporation (http://www.cfdrc.com/datab/Applications/MEMS/mems.html) IntelliSuite (http://www.intellisuite.com/) 
MEMCAD (http://www.memcad.com)

FlumeCAD(http://www.flumecad.com)

SESE (http://www.nmtec.ch)

Solidis (http://www.ise.ch)

Coyote Systems (http://www.coyotesystems.com/applications/applications.html)

IntelliSense Corporation (http://www.intellisense.com/software.html)

Microcosm Technologies, Inc. (http://www.memcad.com/products.html)

Stanford University (http://www-tcad.stanford.edu/tcad.html)

University of Illinois (http://galaxy.ccsm.uiuc.edu/mems_research.htm)

Reduced Order Modeling

Analogy (http://www.analogy.com/Mixed/default.htm)

Duke University (http://www.ee.duke.edu/Research/IMPACT/)

Massachusetts Institute of Technology (http://rle-vlsi.mit.edu/research)

Microcosm Technologies, Inc. (http://www.memcad.com/products.html)

System Level Modeling

Carnegie Mellon University (http://www.ece.cmu.edu/ mems/projects/memsyn/index.sht $\mathrm{ml}$ )

University of California-Berkeley (http://ptolemy.eecs.berkeley.edu/)

Tool Suites

MEMSCAP (http://www.memscap.com/)

An's MEMS CAD, http://myhome.dreamx.net/piyo123/default.html

IntelliSense (2001), http://www.intellisense.com/

Jess (1999), http: // herzberg.ca.sandia.gov /jess, Sandia National Laboratories

MEMCAD (2000), http://www.memcad.com/

Other

University of California-Berkeley (http://www-bsac.eecs.berkeley.edu/ cfm/)

NIIIP (1999), http:// www.niiip.org/

RaDEO (1998), http://elib.cme.nist.gov/radeo/

Anis, http://www.intellisense.com/software/anise.html

SIMODE, http://www.gemac-cheminitz.de/mst/simode.html

MicroCAD, http://www.fuji-rico.co.jp/crab/electric/semicon/microcad/micro.html

SEGS, http://www.design.caltech.edu/Research/MEMS/software.html

SIMPler, http://www-inst.eecs.berkeley.edu/ ee40/SIMPLer/SIMPLer.html

CaMEL, http://www.memsrus.com/cronos/svcs2tcml.html

LASI, http://cmoseu.com/cmos1/winlasi/winlasi.htm

MAGIC, http://www.research.digital.com/wrl/projects/magic/magic.html

VALCAIN, http://www.memscap.com

MEMS Pro, http://www.tanner.com or http://www.memscap.com

Mentor Graphics, http://www.mentorg.com

Saber, http://www.analogy.com 
3

GaAs Thermally Based MEMS Devices-Fabrication Techniques, Characterization and Modeling MEMS Device Design and Fabrication

MEMS Device Thermo-Mechanical Characterization

MEMS Device Thermo-Mechanical Modeling

Tibor Lalinský1 ${ }^{1}$, Milan Držík ${ }^{2}$, Jiří Jakovenko ${ }^{3}$, and Miroslav Husák ${ }^{3}$

${ }^{1}$ Institute of Electrical Engineering, Slovak Academy of Sciences, Dúbravska cesta 9, 84104 Bratislava, Slovakia

${ }^{2}$ International Laser Center, Ilkovičova 3, 81219 Bratislava, Slovakia

${ }^{3}$ Czech Technical University, Dept. of Microelectronics, Technicka 2, 16627 Prague 6,

Czech Republic

MEMS Device Design and Fabrication

\section{INTRODUCTION}

Silicon (Si) based MicroElectroMechanical Systems (MEMS) are now well understood and widely used in various integrated micromachined microsensors and microactuators. In relation to this, gallium arsenide (GaAs) offers a number of material-related properties and 
technological advantages over $\mathrm{Si}$ [1-3]. These include well know properties, such as direct band gap transition and high electron mobility.

A very important feature of GaAs is the possibility of forming compatible ternary and quaternary compounds by alloying. Using GaAs as a substrate material, formation of $\mathrm{Al}_{x} \mathrm{Ga}_{1-x} \mathrm{As}$ is especially attractive, since their lattice constants are nearly equal, and aluminum and gallium atoms are easily substituted in the lattice without causing too much strain in the film. Thanks to this prominent feature, a number of interesting properties and phenomena, such as high-mobility two-dimensional carrier gases, resonant tunneling, and fractional quantum Hall effect, have been found in the $\mathrm{Al}_{x} \mathrm{Ga}_{1-x} \mathrm{As} / \mathrm{GaAs}$ heterostructure system. New devices, such as modulation-doped FETs, heterojunction bipolar transistors, hot electron transistors, resonant tunneling transistors, quantum-well lasers, and other photonic and quantum effect devices, have been developed using this heterostructure system. The heterostructure system seems to be of primary importance also for the investigation of new one- and zero-dimensional effects in structures, such as quantum wires and dots. These areas are recognized to be very interesting from the viewpoint of semiconductor physics and device engineering.

GaAs also has piezoelectric properties comparable with those of quartz. The piezoelectric response of GaAs is an attractive feature. It gives the possibility of activating motions by an electric field and of detecting motions by bound charges generated by the mechanical stress [4]. Other advantages with piezoelectricity are negligible thermal gradients due to the low activation power (of the order of $\mu \mathrm{W}$ ), and the possibility of detecting very small mechanical amplitudes. The response time of the piezoelectric effect is limited by Maxwell's equations and, therefore, it is extremely small. In general, the response time is restricted by mechanical damping of the structure. The piezoelectric effect in GaAs is well suited for resonant sensors and actuators.

GaAs also exhibits a very interesting piezoresistive response [2]. In GaAs, the physical mechanisms that change the resistance due to an applied stress are different from those in silicon. One response mechanism is the observed mobility change due to the change of the electron effective mass with pressure. Another mechanism is a pressure induced transfer of electrons from the high-mobility band gap minimum $\Gamma$ to low mobility minimum $\mathrm{X}$ or $\mathrm{L}$ due to a change of their relative energy. The third response mechanism is the pressure induced freezing of electrons to deep level impurity states DX, observed mainly for $\mathrm{Al}_{x} \mathrm{Ga}_{1-x} \mathrm{As}$ epitaxial layers of compositions about $x=0.3-0.35$. The fourth response mechanism is somewhat different from the others, using the stress gradient induced piezoelectric bound charges to change the resistivity in a diffused resistor. Combination of piezoresistive and piezoelectric effects in a GaAs two-dimensional electron gas (2DEG) layer yields a higher effective piezoresistive coefficient compared with $\mathrm{Si}$ [5]. The piezoresistive coefficient of GaAs with $2 \operatorname{DEG}\left(\Pi_{\mathrm{L}}=46 \times 10^{-10} \mathrm{~m}^{2} / \mathrm{N}\right)$ is almost 10 times higher than that of $\mathrm{Si}\left(\Pi_{\mathrm{L}}=\right.$ $\left.5.7 \times 10^{-10} \mathrm{~m}^{2} / \mathrm{N}\right)$.

Further GaAs response mechanisms, such as thermoresistive, piezooptic and direct band gap responses, may also be found in [2].

$\mathrm{GaAs}$ is also an attractive material for thermal sensors because it can operate at ambient temperatures up to $350{ }^{\circ} \mathrm{C}$ thanks to its wide band gap. The higher thermal resistivity and higher Seebeck coefficient, as compared with $\mathrm{Si}$, make it a very promising material for such MEMS devices as microwave power sensors, gas sensors and flow sensors. Using various micromachining techniques it is possible to fabricate free-standing micromechanical 
structures that are thermally isolated from the bulk material, allowing realization of thermal based MEMS devices.

Likewise, the initial MBE and MOCVD grown GaAs heterostructures provide more flexibility and precision in micromachining, which results in very sharp interfaces (one or two monolayers). Due to different compositions, these layers can easily be etched by wet or dry etching techniques with excellent compositional selectivity. Precise control of the thickness and uniformity of GaAs based micromechanical structures like cantilever beams $[3,6,7]$, membranes or bridges $[1,8-11]$ can be achieved directly via the thickness of the MBE grown materials over an etch stop layer.

Additional advantages can also be taken by the well-controlled mechanical characteristics offered by single-crystalline epitaxial layers. The measured fracture properties of GaAs are known to be sufficiently good with average fracture strength of $2.7 \mathrm{GPa}$ (i.e., at least three times as high as that of most construction steels) $[1,2]$.

Technological advantages and intrinsic physical properties of GaAs heterostructures have been demonstrated in various MEMS based on GaAs solid state device and micromechanical structure technology [1, 3, 9-18]. Most of them were designed for application as micromachined microsensors for electrical power sensing [3, 12-16], infrared thermal radiation detection [18] and for pressure sensing [10, 11].

In order to demonstrate the high resistivity and micromachining capabilities of GaAs, micromachined coplanar waveguides have been developed $[17,19]$ to suppress highfrequency losses at millimeter and submillimeter wavelengths. Moreover, a suspended planar spiral inductor and a micromachined GaAs/metal thermopile [1], and a suspended GaAs resistor [9] as special micromechanical devices have also been demonstrated for GaAs MEMS design. The introduction of new material families, such as AlGaAs/GaAs, offered compatibility of the micromechanical devices with the MESFET or pHEMT technology, so that they can be integrated with GaAs based monolithic microwave integrated circuits (MMICs).

A GaAs cantilever has also been introduced as a micromechanical device for fabrication of novel probes for scanning probe microscopy (SPM). Recently, the developments in the field of micromachined technology of GaAs cantilevers with integrated tips like those of atomic force microscopy (AFM) cantilevers have been successfully reported [20, 21]. These cantilevers represent the basic sensor design for both passive as well as active scanning near field probes. For example, using an integrated Schottky diode as a temperature sensor and photodetector in a GaAs tip, great potentials for the development of scanning thermal microscopy (SThM) and scanning near-field optical microscopy (SNOM) can be predicted. Likewise, the combination of standard III-V based epitaxy techniques (MBE or MOCVD) with 3D growth of different layer heterostructures for light-emitting and laser diodes or quantum dots on the tip is a challenge for future new concepts of GaAs based Micro(Nano)OptoElectroMechanical System-M(N)OEMS devices.

In addition, the bimetallic effect was observed in $2 \mu \mathrm{m}$ thick GaAs cantilever beams of a power sensor microsystem [14, 22, 23]. Cantilever deflections were induced by differential thermal expansion of the cantilever layers using GaAs MESFET as a heater.

Thermal actuation of suspended GaAs membrane Bragg reflectors has been performed to tune the Fabry-Pérot filters [24]. Four supporting membrane bridges have been used with resistor heaters as driving elements. Thermal actuation of the membrane yielded a mechanical sensitivity of $13 \mathrm{~nm} / \mathrm{mW}$ under normal pressure. 
TABLE 1. Structural, thermal and mechanical properties of Si, GaAs and AlAs [2]

\begin{tabular}{lccc}
\hline Semiconductor materials & $\mathrm{Si}$ & $\mathrm{GaAs}$ & AIAs \\
\hline Crystal structure & Diamond & Zincblende & Zincblende \\
Lattice constant, a $(\AA)$ & 5.4311 & 5.6533 & 5.6611 \\
Density, $\rho\left(10^{3} \mathrm{~kg} \mathrm{~m}^{-3}\right)$ & 2.3290 & 5.360 & 3.760 \\
Melting point, $\mathrm{T}_{\mathrm{M}}\left({ }^{\circ} \mathrm{C}\right)$ & 1413 & 1238 & 1740 \\
Specific heat, $\mathrm{C}_{\mathrm{p}}\left(\mathrm{J} \mathrm{g}^{-1} \mathrm{~K}^{-1}\right)$ & 0.71 & 0.35 & 0.48 \\
Thermal resistivity, $\mathrm{W}\left(\mathrm{K} \mathrm{cm} \mathrm{W}^{-1}\right)$ & 0.64 & 2.27 & 1.1 \\
Thermal expansion coefficient, $\alpha_{11}\left(10^{-6}{ }^{\circ} \mathrm{C}^{-1}\right)$ & 2.6 & 6.4 & 5.2 \\
Debay temperature, $\theta_{\mathrm{D}}(\mathrm{K})$ & 463 & 370 & 446 \\
Fracture toughness, $\mathrm{K}\left(\mathrm{MPa} \mathrm{m}^{1 / 2}\right)$ & 0.9 & 0.44 & 1.7 \\
Hardness, $\mathrm{H}_{\mathrm{v}(100)}(\mathrm{GPa})$ & 10 & 7 & 5 \\
Stiffness constants $(\mathrm{GPa})$ & & & \\
$c_{11}$ & 165.6 & 118.8 & 120.2 \\
$c_{12}$ & 63.98 & 53.8 & 57.0 \\
$c_{44}$ & 79.51 & 58.9 & 58.9 \\
Elastic compliance constants $\left(10^{-12} \mathrm{~Pa}^{-1}\right)$ & & & \\
$s_{11}$ & 7.7 & 11.7 & 12 \\
$s_{12}$ & -2.1 & -3.1 & -3.9 \\
$s_{44}$ & 12.6 & 16.8 & 17 \\
Piezoelectric coefficient $\mathrm{d}_{14}(\mathrm{pm} / \mathrm{V})$ & 0 & -2.69 & -3.82 \\
\hline
\end{tabular}

A comprehensive electro-mechanical performance analysis of a thermally actuated GaAs cantilever was also reported [25, 26]. An integrated GaAs MESFET heater was used to actuate a $2 \mu \mathrm{m}$ thick GaAs cantilever. Simultaneously, a Schottky gate diode of a MESFET was used to sense the cantilever temperature corresponding to various levels of power dissipation. The analyzed device has shown an excellent linearity in the electro-thermomechanical conversion characteristics and high electro-mechanical conversion efficiency $\left(\Gamma_{\mathrm{E}}=802 \mathrm{~nm} / \mathrm{mW}\right)$. The dynamic behavior of the GaAs cantilever was tested with a square wave signal. The mechanical time constant of $1.4 \mathrm{~ms}$ obtained from the measured time response of the cantilever deflection was found to be limited by the electro-thermal time response. The resonant frequency of the cantilever as high as $15.44 \mathrm{kHz}$ was measured.

Based on this short review devoted to design and fabrication of various micromachined GaAs based devices and structures it should be noted that both GaAs and AlGaAs materials seem to be the basic electronical and construction materials for MEMS design, mainly thanks to their structural, electrical and thermo-mechanical properties. The basic structural, electrical, mechanical and thermal properties of both materials have been well-introduced by many of authors. They can be found, for example, in [2, 27-32]. For comparison, some of their properties are also summarized in Table 1 [2].

In this chapter, various GaAs micromachining approaches compatible with GaAs heterostructure based device fabrication techniques are introduced. Special attention is given to GaAs thermally based MEMS devices because they directly demonstrate the necessity for mutually compatible integration of the micromechanical structures with the MESFET or HEMT high-speed devices. Efforts are made to cover the basic fabrication technologies, techniques, processes, and materials. One of the main goals is to show how to integrate GaAs microelectronic devices with GaAs micromechanical structures in order to produce integrated high performance MEMS. This chapter introduces the mentioned group of MEMS devices in all their complexity and multidisciplinary basis. Therefore, besides 
fabrication methods, non-conventional optical methods are introduced to analyze the basic thermo-mechanical properties of the MEMS devices. They permit (in situ) to study three-dimensional (3D) device nano-deformations induced by temperature changes in both stationary and non-stationary dynamic process conditions. Finally, various approaches and design concepts in the field of 3D thermo-mechanical modeling and simulation are also introduced and directly compared with comprehensive experimental electro-thermo-mechanical characterization of the MEMS devices. In general, these model approaches demonstrate a great potential for studying the thermo-mechanical phenomena and effects observed in MEMS devices on the required micro- and nanoscale. They are considered to be indispensable for the design of novel 3D GaAs thermally based MEMS devices.

\section{DESCRIPTION OF GaAs THERMALLY BASED MEMS}

A GaAs thermally based MEMS integrates GaAs microelectronic devices such as high-speed transistors or resistors, and temperature sensors on GaAs thermally isolated micromechanical structures, such as membranes, cantilevers and bridges. The microelectronic devices are designed to serve as micro-heaters, and the temperature sensors are proposed "in situ" to sense the temperature of the micromechanical structures.

MEMS, as described above, fulfils the requirements imposed on the so-called micromachined thermal converter (MTC) device. The main design criterions, such as high electro-thermal conversion efficiency, linearity, short response time, thermal stability, micromechanical integrity and integration device simplicity should be taken into account in the MTC design.

Due to a higher thermal resistivity and operation at high temperatures (see Table 1), MTC based on GaAs should be able to perform electro-thermal conversion with a higher conversion efficiency than Si. Likewise, the high-speed performance of GaAs based fieldeffect transistors (MESFET, HFET, HEMT) creates conditions for the design of MEMS in monolithic microwave integrated circuit design. GaAs based MTC devices can be considered to be a "heart" of various thermal based MEMS, such as microwave power sensors, infrared thermal sensors (infrared bolometers), gas sensors, vacuum sensors, micromechanical wind flow sensors, thermally actuated microactuators, and even accelerometers.

Fig. 1 shows a schematic view of a GaAs bridge based MTC device. As it has already been mentioned, it consists of a micro-heater and a temperature sensor monolithically integrated on a thin GaAs bridge structure. To fabricate such a device, the front-side processing

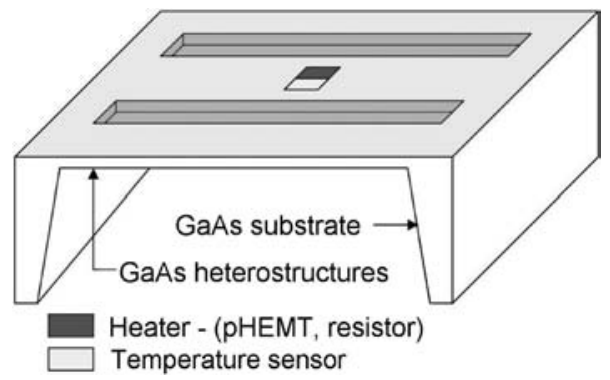

FIGURE 1. Schematic view of GaAs bridge based MTC device. 
technology of the micro-heater and temperature sensor must be combined with surface and bulk micromachining of GaAs. As a rule, GaAs micromachining technology should be fully compatible with the processing technology of integrated microelectronic devices. Therefore, in the next parts of this chapter the basic GaAs micromachining techniques and MEMS fabrication technologies are described in detail.

\section{GaAs MICROMACHINING TECHNIQUES}

In GaAs micromachining, wet and dry etching processes are widely used. Wet etchants are categorized as isotropic etchants (attack the material being etched at the same rate in all directions) and anisotropic etchants (attack the material or GaAs at different rates in different directions, and therefore, shapes/geometry can be precisely controlled). In other words, the isotropic etching has a uniform etch rate at all orientations, while for anisotropic etching, the etch rate depends on crystal orientation.

The dry etching process often used in GaAs micromachining is reactive ion etching (RIE). In this process, ions are accelerated towards the material to be etched, and the etching reaction is enhanced in the direction of ion trajectory. Reactive ion etching is therefore an anisotropic etching process.

Selectivity of the etching process is the most important property utilized in GaAs micromachining. It is defined as a ratio between the structural material (slower etching) and sacrificial material (faster etching) etch rates for a specific etchant. For a few systems, entirely selective etching can be achieved allowing real etch stop materials. The most frequently used sacrificial wet and dry etch systems for III-V compound heterostructures are presented in $[33,34]$. It can be noted that not only different materials but also different dopant concentrations and damaged regions could act as stop and sacrificial layers.

A great number of micromachining techniques have already been developed for GaAs, such as selective etch stops for hetero- and homostructures of various electrical properties, sacrificial layer techniques with various etch rate selectivities, and dry and wet etching for isotropic and anisotropic shaping.

Two main methods can be applied to fabricate GaAs micromechanical structures: bulk micromachining, where structures are etched in the substrate, and surface micromachining, where micromechanical layers are formed from layers deposited onto the surface. In bulk micromachining, two different approaches have been considered: etching from the front side and from the back side of the wafer.

\section{Front Side Bulk Micromachining}

In the case of front side bulk micromachining, sacrificial damaged GaAs layers created by high energy ion implantation of nitrogen can be used [35, 36]. Energy ions effectively produce a lot of damage in GaAs. The displaced atoms in the damaged regions are chemically less stable than the original GaAs atoms. If a layer has a sufficient density of displaced atoms, it can be used as a sacrificial layer, selectively removable by defect-sensitive etchants. Deep low-dose nitrogen implantation can also compensate n-doped GaAs to n-GaAs at the top, which then can be used as an etch stop layer if electrolytic etching techniques are applied. On 


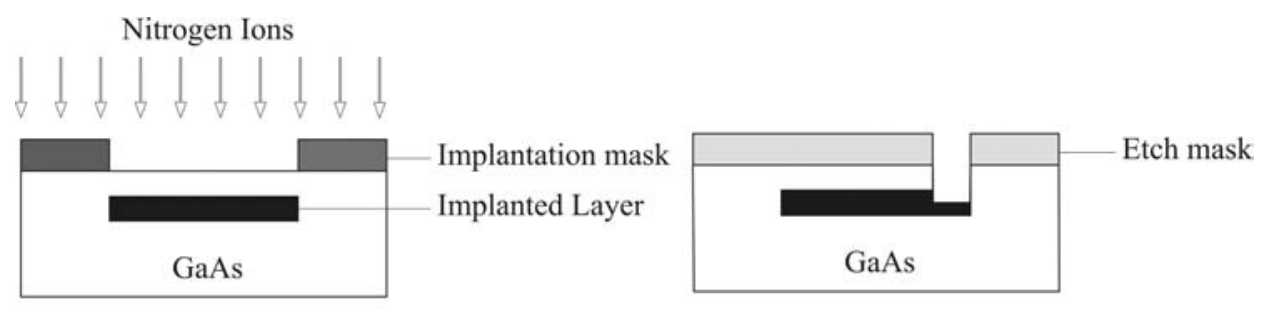

(a)

(b)

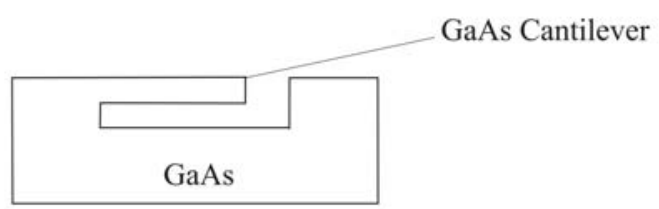

(c)

FIGURE 2. Fabrication of a GaAs cantilever using wet chemically selective etching: (a) selective implantation, (b) annealing and non-selective etching, (c) selective etching [36].

the other hand, deep high-dose implantation of nitrogen into GaAs followed by subsequent annealing produces buried $\mathrm{GaAs}_{1-x} \mathrm{~N}_{y}(y<x<1)$ layers which can be used as sacrificial layers and are selectively etched in $1 \mathrm{~N} \mathrm{NaOH}$ solution.

Fig. 2 schematically describes the fabrication steps of the GaAs cantilever [36], starting with selective ion implantation of nitrogen (Fig. 2a), followed by post-implant annealing and masked nonselective etching of the top n-GaAs layer (Fig. 2b), and ending by selective etching of the buried $\mathrm{GaAs}_{1-x} \mathrm{~N}_{y}$ layer (Fig. 2c).

A front side $\mathrm{AlGaAs} / \mathrm{GaAs}$ micromachining technique that is compatible with the laser diode fabrication process has been described [37]. AlGaAs structural layers and GaAs sacrificial layers were prepared by metal organic vapor phase epitaxy (MOVPE). Reactive dry etching with chlorine in combination with selective etching of the GaAs sacrificial layer in peroxide/ammonium hydroxide solution were used to fabricate high-aspect structures. Fig. 3 shows the fabrication process of an $\mathrm{AlGaAs}$ microcantilever. First, the AlGaAs structural layer is grown by MOVPE. Then, after making the resist pattern for the cantilever, the substrate is etched vertically through the $\mathrm{AlGaAs}$ etching stop layer by reactive fast atom beam etching (RFAB). Chlorine served as an etching gas, and a hard-baked photoresist $\left(160^{\circ} \mathrm{C}\right)$ as an etching mask. Finally, the GaAs layer underneath the cantilever was selectively removed by a peroxide/alkaline (P/A) system of $30 \% \mathrm{H}_{2} \mathrm{O}_{2}$ and $29 \% \mathrm{NH}_{4} \mathrm{OH}$ in order to make a movable structure. The volume ratio of the etchant has the maximum selectivity of 70 for $\mathrm{Al}_{0.6} \mathrm{Ga}_{0.4} \mathrm{As}$ used in the experiment.

Front side bulk micromachining has also been used to fabricate free-standing triangular prism-shaped bridges for $\mathrm{GaAs}$ thermocouple applications using a $\mathrm{H}_{2} \mathrm{SO}_{4}$-based etchant [38]. Such a kind of structure has been investigated in detail in [39, 1, 9], using the micromachining technique adopted at TIMA-CMP laboratory as a broker for a number of technologies (prototyping and low volume production) [40]. The strategy adopted at TIMA-CMP laboratory consists in applying front side bulk micromachining in standard IC processes, keeping superposed opening regions in the dielectric layers to access 


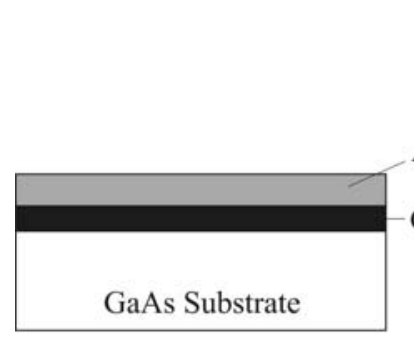

(a)

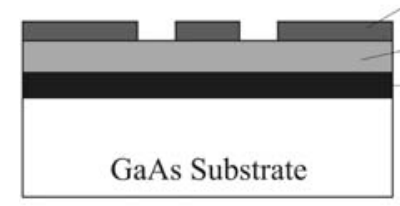

(b)

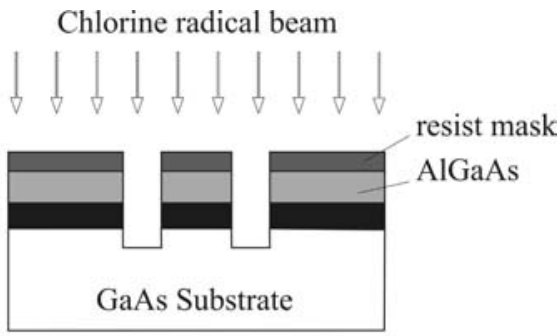

(c)

resist mask

$\mathrm{AlGaAs}$

GaAs buffer layer

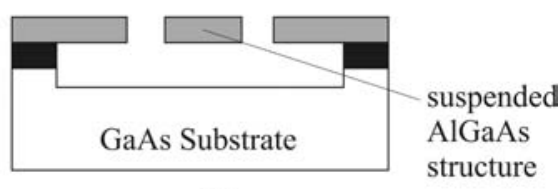

(d)

FIGURE 3. Fabrication process of an AlGaAs cantilever: (a) epitaxial growth, (b) photoresist patterning and baking $\left(160{ }^{\circ} \mathrm{C}\right)$, (c) reactive dry etching by chlorine plasma through AlGaAs etching stop layer, (d) GaAs layer removal by P/A-30 [37].

the substrate surface. The structures are then released through additional post-process wet etching. For the suspended GaAs/AlGaAs mesa-shaped bridge structure depicted in Fig. 4, a mesa structure used to fabricate HEMT transistor is placed between the two open areas. During post-process etching, $\mathrm{AlGaAs}$ acts as a stop layer to keep the top GaAs epilayer, while the GaAs substrate as well as the InGaAs layer are selectively removed. Thus, the etching selectivity of GaAs to AlGaAs is the critical parameter to be controlled. For example, considering an AlGaAs thickness of $500 \AA$, selectivity 100 and a symmetrical bridge, the maximum mesa width over the bridge must be $5 \mu \mathrm{m}$ before starting the etching of the top GaAs layer. This kind of bridge structure allows to suspend GaAs resistors useful to realize bolometers, piezoresistive-based sensors or metal/semiconductor thermocouples

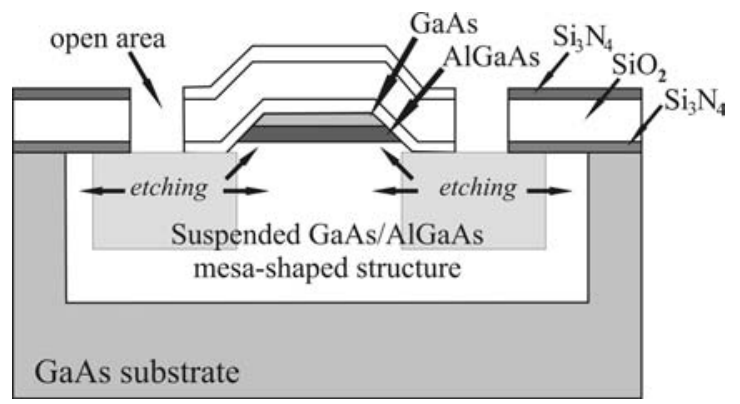

FIGURE 4. Suspended GaAs/AlGaAs mesa-shaped bridge structure [9, 39]. 


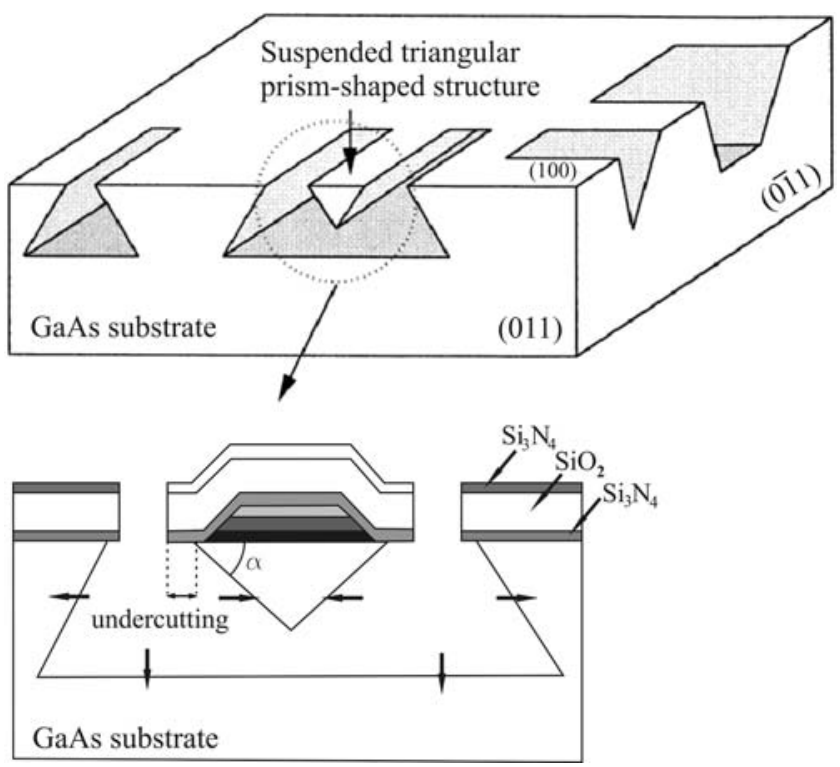

FIGURE 5. Free standing triangular prism-shaped bridge structure $[9,39]$.

for infrared thermopile sensors. The triangular prism-shaped bridge structure illustrated in Fig. 5 can be obtained via selective anisotropic etching. Experimental details of the etching process can be found in $[9,39]$. This type of bridge structure is suitable for fabrication of free-standing active devices (diodes and transistors) or thermal sensors, as mentioned later on.

\section{Back Side Bulk Micromachining}

The back side bulk micromachining commonly involves stopping on an epitaxial layer. Additional masking on the back side and special alignment techniques are required. Because of the high selectivity of the GaAs/AlGaAs system, it is possible to etch several hundred micrometers of GaAs, stopping at an exact depth defined by a stop layer of AlGaAs. This technique has been widely used to fabricate GaAs membranes $[10,11,15,16]$, cantilevers $[3,6,7,12-14]$ and bridges [19].

Fig. 6 shows a cross-section through a GaAs membrane patterned by the back side wet chemical etching technique [10]. Definition of the membrane was done by using fast etching over $400 \mu \mathrm{m}$. An unselective etchant $\mathrm{H}_{2} \mathrm{SO}_{4}: \mathrm{H}_{2} \mathrm{O}_{2}: \mathrm{H}_{2} \mathrm{O}$ with concentration ratio 4:3:3 was used in the bubble etch method. Then etching continues with selective etchant $\mathrm{NH}_{4} \mathrm{OH}: \mathrm{H}_{2} \mathrm{O}_{2}$ with concentration ratio 1:24. Etching continues until the AlAs etch stop layers are reached. This isotropic etchant has a selectivity of 1000 and $\mathrm{pH}$ around 8 . The AlAs etch stop is removed using diluted HF which has a selectivity of $10^{7}$. A simplified description of such a process is also depicted in Fig. 7 [11]. The back side etch mask is 


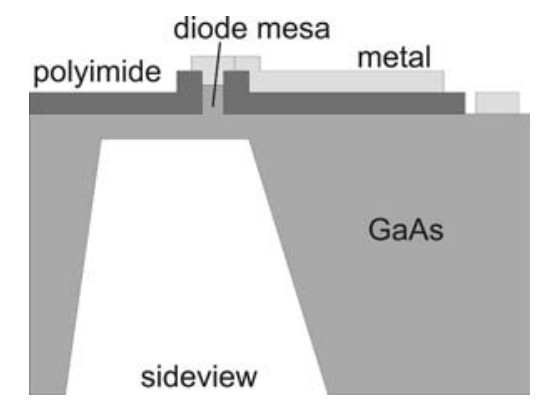

FIGURE 6. A cross-section through GaAs membrane patterned by back side wet chemical etching technique [10].

patterned in the first step. Then, fast unselective etching is performed, followed by slower selective etching, stopping at the $\mathrm{AlGaAs}$ layer.

Besides the mentioned wet etching techniques, anisotropic dry etching techniques have also been developed for back side bulk GaAs micromachining [3, 6, 7, 12]. The etching experiments were aimed at establishing the etch conditions which would best meet the following process requirements:

- sufficient etch rate of GaAs for deep back side RIE through a GaAs substrate to the etch stop layer, with anisotropy needed to minimize lateral undercutting,

- selectivity of GaAs etching to the etch masks to achieve back side over-etching of the GaAs substrate to the stop layer,

- selectivity of GaAs to AlGaAs etching needed for smooth bottom of the back side etched cantilevers.

$\mathrm{CCl}_{2} \mathrm{~F}_{2}$ was used as the process gas, the chamber pressure during etching being 10, 20 and $30 \mathrm{~Pa}$ at a gas flow of $30 \mathrm{sccm}$. The RF power was varied from 50 to $150 \mathrm{~W}$. Fig. 8 shows the basic fabrication steps of $2 \mu \mathrm{m}$ thick GaAs cantilevers [7]. The GaAs/AlGaAs heterostructure layer system grown by MBE was designed to be used for both GaAs MESFET technology and micromechanical structures fabrication. In the first step, double-side aligned
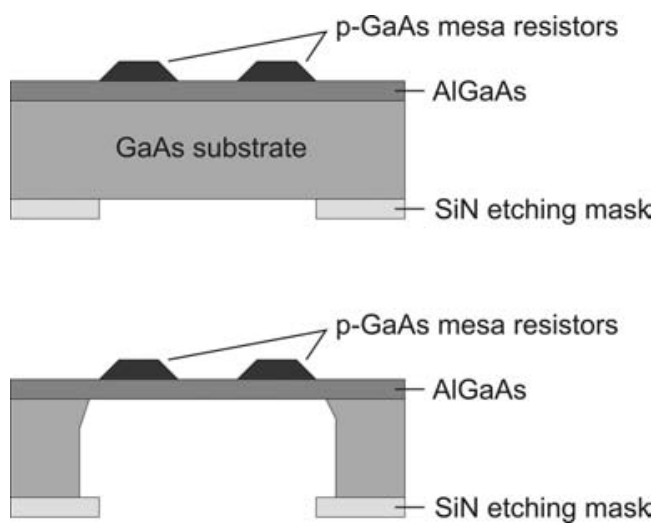

FIGURE 7. Back side GaAs bulk micromachining using AlGaAs as etch stop layer [11]. 

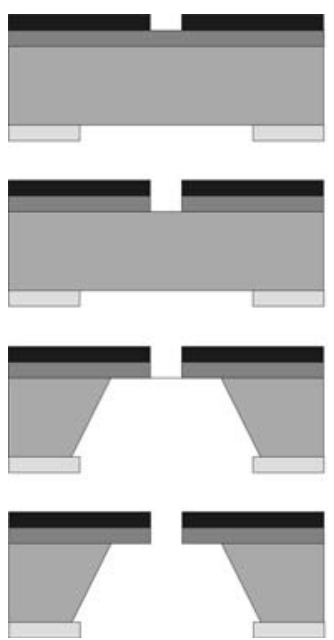

Patterning of masks

on both sides of

substrate

High selective front side

RIE of GaAs using

$\mathrm{CCl}_{2} \mathrm{~F}_{2}$ process gas

High selective back side

RIE of GaAs using

$\mathrm{CCl}_{2} \mathrm{~F}_{2}$ process gas

Selective etching of

AlGaAs stop layer

FIGURE 8. Fabrication steps of GaAs cantilevers by back side dry plasma etching technigue [7].

photolithography is carried out to define the etching masks on both sides of the substrate. After this, highly selective RIE of GaAs from the front side was used to define the lateral dimension of the cantilever. The vertical dimension of the cantilever was defined by deep back side RIE through a $350 \mu \mathrm{m}$ thick GaAs substrate to the AlGaAs etch-stop layer, hence the cantilever thickness (vertical dimension) is precisely determined by the thickness of MBE grown layers over this etch stop layer. The last step is selective etching of the AlGaAs stop layer.

Recently, a novel bulk GaAs micromachining technology has been introduced [41, 42]. It permits to fabricate very thin GaAs heterostructure based cantilevers and bridges fully compatible with the processing technology of GaAs pHEMT. In this technology a thin polyimide membrane with a low mechanical stress and thermal conductivity is used for mechanical fixing and thermal insulating of micromechanical structures. The micromachining technology, in principle, can also be used to define very thin small-size thermally isolated islands or dot areas. The process flow is schematically shown in Fig. 9. In order to demonstrate the process compatibility of the pHEMT with the micromachining technology of micromechanical structures, the technology starts with an MBE or MOCVD growth of GaAs heterostructures, which defines both the pHEMT device and the thickness of the micromechanical structures. Then, a front-side processing technology is performed to define Source (S), Drain (D) and Gate (G) contacts of the pHEMT. The next step is surface micromachining of a cantilever, bridge or island by masked non-selective wet or plasma etching. In this step the whole layer structure has to be etched away up to SI GaAs substrate. Surface micromachining is followed by deposition and subsequent thermal forming of a thin top polyimide layer. Finally, three-dimensional patterning of the micromechanical structures is defined by deep back side selective reactive ion etching of SI-GaAs through the openings in the mask, using $\mathrm{AlGaAs}$ or InGaP as etch-stop layers. It should be emphasized that in this deep back side etching the polyimide layer also fulfils the role of the additional back side etch stop layer. After bulk GaAs micromachining, it enables the micromechanical structures to be mechanically fixed and thermally isolated in space. 


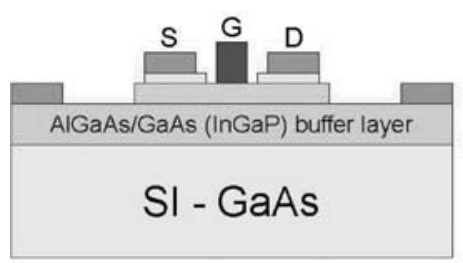

(a)

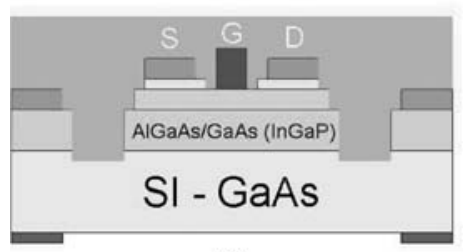

(c)

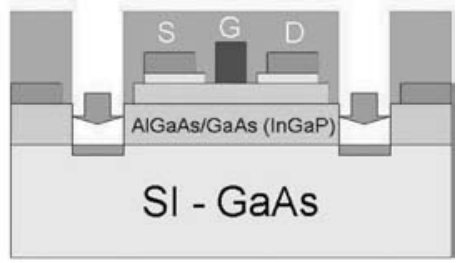

(b)

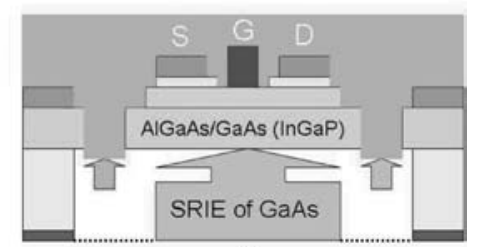

(d)

FIGURE 9. Fabrication steps of polyimide fixed cantilever and bridge structures by back side dry plasma etching technique: (a) HEMT layer growth by MBE or MOCVD, (b) surface micromachining of cantilever or bridge structures, (c) polyimide layer deposition and thermal forming, (d) bulk micromachining of GaAs substrate $[41,42]$.

The micromachining technology permits precise control of the thickness and uniformity of the cantilever, bridge or island structures directly by the thickness of MBE or MOCVD grown buffer layers. Moreover, it permits to define islands and dot areas wherever in space, keeping the thermal resistance value as high as possible.

\section{TEMPERATURE SENSORS}

As it was already discussed in the previous part of this chapter, temperature sensors are becoming an integral part of thermally based MEMS devices, where they are designed to serve as low power and high precision temperature sensing elements. They convert the temperature or temperature difference directly into an electrical signal.

For the design of GaAs thermally based MEMS devices, the following kinds of temperature sensors are used:

- thin film temperature sensor,

- Schottky diode temperature sensor,

- metal/GaAs thermocouple.

$\mathrm{Ni}$ and Pt thin films were designed and tested as temperature sensing elements on GaAs because of their high temperature coefficients and compatibility with GaAs-based devices and related IC processing steps [43]. In the GaAs device technology, mostly metal/GaAs contact systems are involved for alloyed ohmic contact fabrication (AuGe/Ni) and Schottky gate barrier formation (Ti/Pt/Au). Both of the thin films, when deposited directly on $\mathrm{GaAs}$, exhibit high chemical reactivity and interdiffusion effects on GaAs interface mainly at elevated temperatures. This can lead to degradation of their electrical and thermal properties as observed in [43]. In order to suppress the interfacial interactions, a thin diffusion barrier layer has to be formed on the GaAs surface before Pt and Ni deposition. 


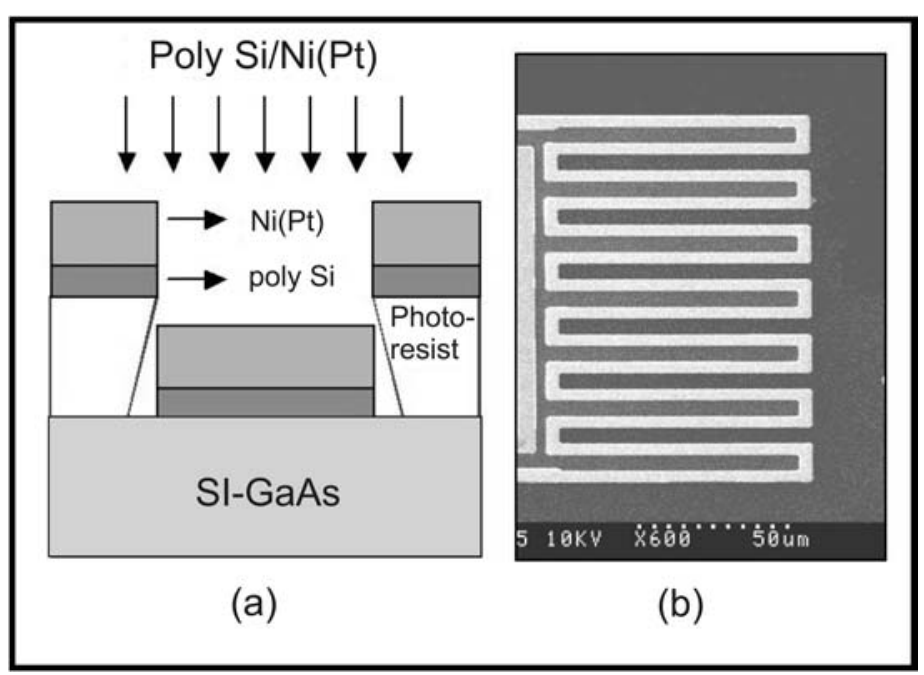

FIGURE 10. Fabrication of thin film temperature sensor, (a) thin films deposition and patterning, (b) SEM view of meander-like structure of temperature sensor [44].

An undoped, highly resistive polysilicon (poly $\mathrm{Si}$ ) thin film has been designed and tested as a diffusion barrier layer for Pt and Ni temperature sensors on GaAs [44]. RF sputtering and electron beam evaporation (EBE) combined with a lift-off technique were used to pattern a meander-like structure of resistance temperature sensors as shown in Fig. 10. A sputtered poly Si barrier layer with various thickness $(0,10,20$, and $30 \mathrm{~nm})$ was immediately followed by EBE of Pt or Ni thin films. The layer thickness of Pt and Ni was chosen to be $60 \mathrm{~nm}$. The meander-like structure of the temperature sensors was designed with a length and width of $1300 \mu \mathrm{m}$ and $3 \mu \mathrm{m}$, respectively.

The optimal thickness of the poly Si interfacial barrier layer was found to be $20 \mathrm{~nm}$. This was evaluated to be sufficient to form a diffusion barrier layer on the GaAs interface. Pt and $\mathrm{Ni}$ temperature sensors with optimum thickness of the poly Si interfacial layer exhibited a high temperature sensitivity $\left(\alpha_{\mathrm{Pt}, \mathrm{Ni}}>2 \Omega / \mathrm{K}\right.$ or $\left.\alpha_{\mathrm{Pt}, \mathrm{Ni}}>1.5 \mathrm{mV} / \mathrm{K}\right)$ and very good linearity even after aging storage tests at temperature $250^{\circ} \mathrm{C}$ for 300 hours as shown in Fig. 11.

High temperature sensitivity and thermal stability, GaAs IC compatibility as well as very good broad temperature linearity of the thin film resistance temperature sensors make them very attractive for a wide range of applications in GaAs thermally based MEMS and MOEMS.

It is a generally known that the Schottky gate diode of the pHEMT, schematically depicted in Fig. 12, can be directly used to sense the temperature with high sensitivity [41]. The forward $I-V$ characteristic of the diode at constant current biasing is used to convert the temperature into voltage. Fig. 13 shows a typical behavior of the gate diode $I-V$ characteristic measured at room temperature with operating point in the forward direction. If the temperature increases, the corresponding diode voltage decreases due to the Schottky gate barrier lowering. Fig. 14 shows the measured voltage responses of the gate diodes to the temperature. Likewise, very good linearity in the diode voltage responses was observed. The extracted diode temperature sensitivities $(-1.15$ and $-1.34 \mathrm{mV} / \mathrm{K})$ are comparable with 


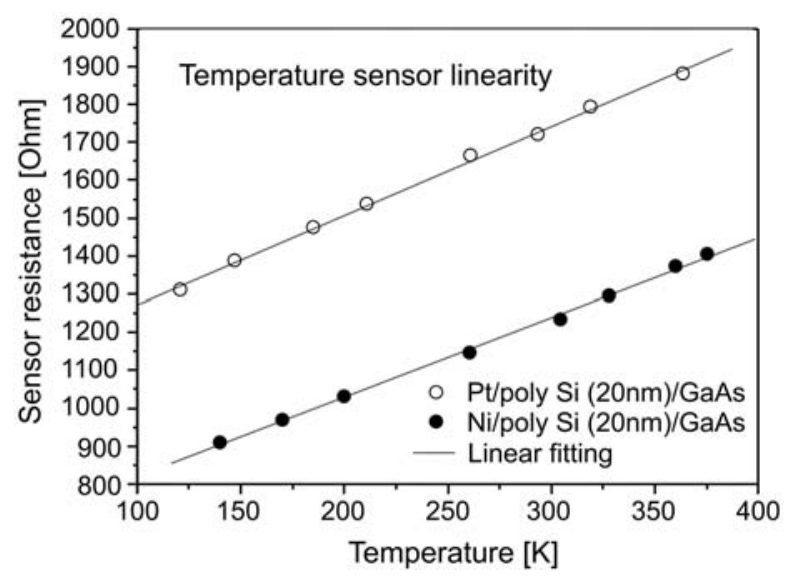

FIGURE 11. Characteristics of Pt and Ni temperature sensors aged at $250{ }^{\circ} \mathrm{C}$ for 300 hours in argon atmosphere [44].

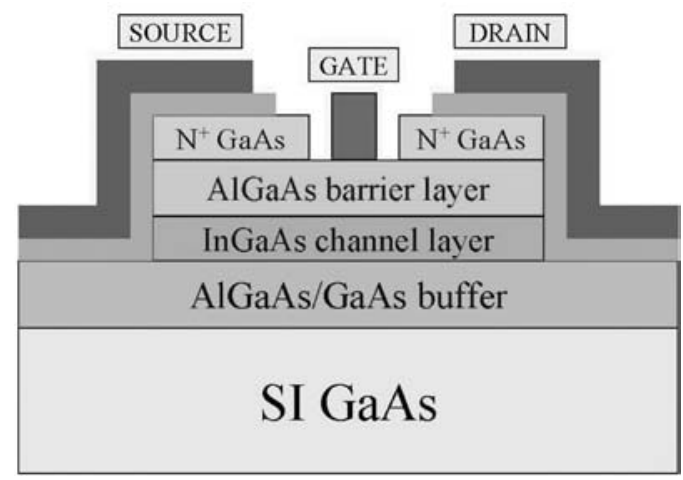

FIGURE 12. Schematic view of Schottky gate diode (source-gate or drain-gate contacts) of HEMT device.

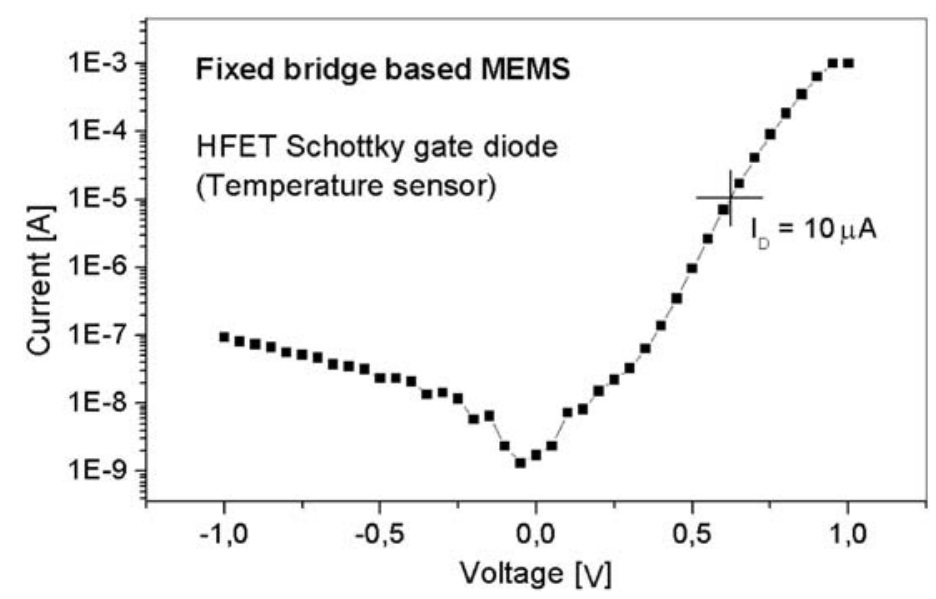

FIGURE 13. Typical behavior of gate diode $I-V$ characteristic measured at room temperature with operating point in the forward direction. 


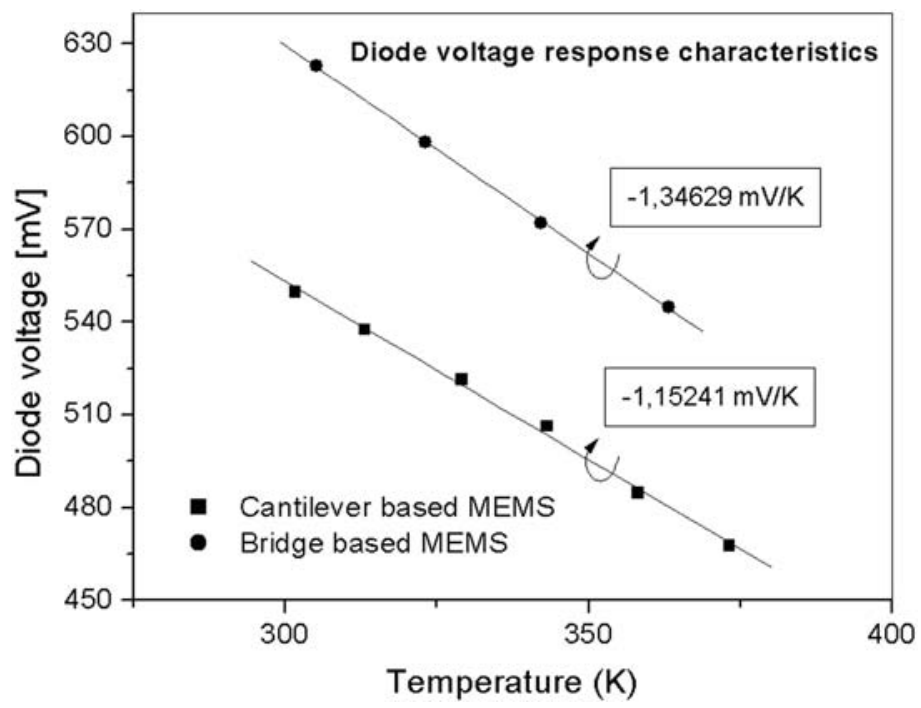

FIGURE 14. Measured voltage responses of gate diodes to the temperature at constant current biasing of $10 \mu \mathrm{A}$ [41].

the Pt or Ni thin film temperature sensors discussed above. However, Schottky gate diode temperature sensors exhibit higher compatibility with the pHEMT processing technology. In contrast to Pt and Ni temperature sensors, no additional lithographic levels are needed because they are directly an integral part of the pHEMT device.

Since semiconductors exhibit a large Seebeck coefficient, great effort has been made to realize on-chip thermopile based sensors using micromachined structures, where the hot junctions are placed at the most isolated portions of the free-standing micromechanical structures and cold junctions over the non-etched substrate region, which acts as a heat sink. Several examples have been presented in Si technologies, using membranes, cantilevers and bridges. Investigations have also been made considering GaAs processing technologies in order to take the advantage of high thermal resistance, higher heat capacitance and higher Seebeck coefficient of GaAs and AlGaAs materials [18, 39, 40, 45-47]. The voltage generated by a thermocouple consisting of two junctions of two different conductors at different temperatures (Seebeck effect) can be increased by connecting a number of thermocouples in series to form a thermopile (Fig. 15). Thermal isolation of the hot junctions by micromachining increases further the performance of such a device $[1,39]$.

A micromachined thermopile structure consisting of $20 \mathrm{GaAs}$-TiAu thermocouples has been efficiently implemented using either triangular prism-shaped bridges (see Fig. 5) or GaAs/AlGaAs mesa-shaped structures (see Fig. 4). It was fabricated using the PML HEMT process $[39,40]$. Details of the design, fabrication and different model approaches can be found in [39]. The micromachined thermopile structure was designed for application in infrared detectors and micromachined electro-thermal converter devices.

The Seebeck coefficient of $\mathrm{Al}_{x} \mathrm{Ga}_{1-x}$ As can vary from 300 to $700 \mu \mathrm{V} / \mathrm{K}$ by changing the $\mathrm{p}$ - or n-type carrier density and the aluminum mole fraction $x[18,48]$. The advantages 


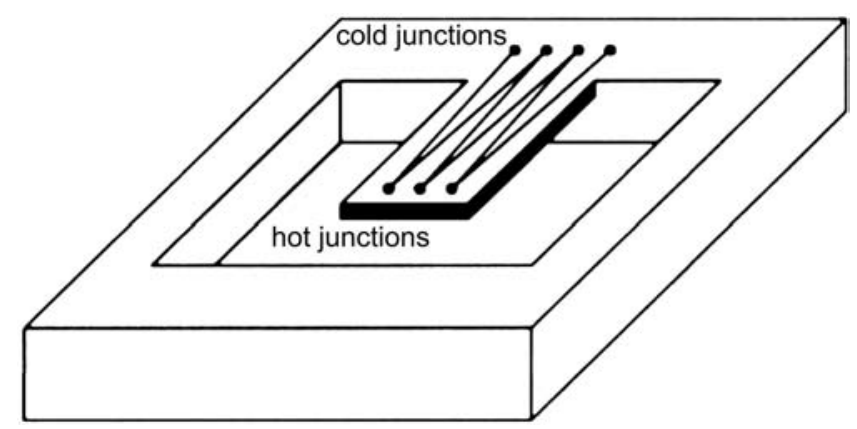

FIGURE 15. Micromachined thermopile structure consisting of hot thermocouple junctions placed on a cantilever structure and cold one placed on a bulk GaAs substrate [39].

of the AlGaAs material system in the design and fabrication of micromachined infrared thermopile sensors were also successfully demonstrated [18].

A schematic cross section through the sensor is shown in Fig. 16. The MOCVD grown $1.2 \mu \mathrm{m}$ thick $\mathrm{Al}_{0.4} \mathrm{Ga}_{0.6} \mathrm{As}$ buffer layer was designed to serve as a supporting membrane and an $\mathrm{n}$-doped $\mathrm{Al}_{0.15} \mathrm{Ga}_{0.85} \mathrm{As}$ channel layer with two different thicknesses was used for thermopiles. A cascade of 20 thermocouples connected in series with $\mathrm{Cr}$-Au interconnectors has been processed with the same masks that are necessary for the MESFET technology. The radiation-induced temperature difference was measured, as seen in Fig. 16. Black-body radiation in the range of $315-530 \mathrm{~K}$ was used to test the sensor sensitivity and detectivity. Sensitivity as high as $145 \mathrm{~V} / \mathrm{W}$ was achieved.

A thermopile structure consisting of 24 series-connected $\mathrm{AlGaAs} / \mathrm{Cr}$-Au thermocouples has also been designed to sense the temperature in micromachined microwave power sensors of sensitivity $\sim 1.6 \mathrm{mV} / \mathrm{K}[15]$.

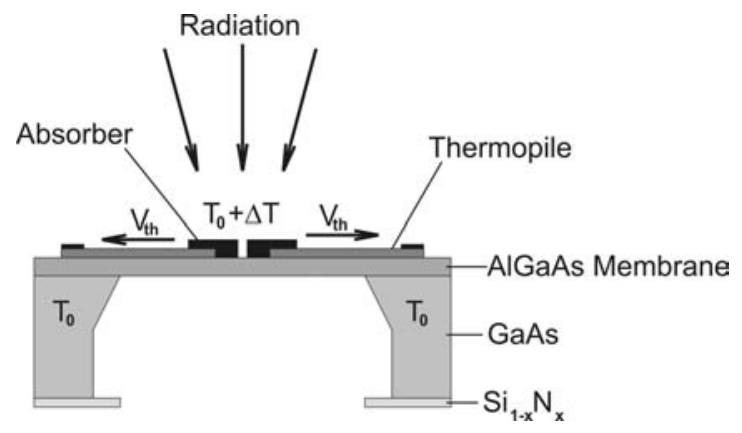

FIGURE 16. Schematic cross section through micromachined infrared thermopile sensors [18].

\section{MICROMACHINED THERMAL CONVERTERS}

MTC devices are widely used for power sensors in a broad frequency range. One of the first micromachined power sensors based on GaAs micromachining technology was 


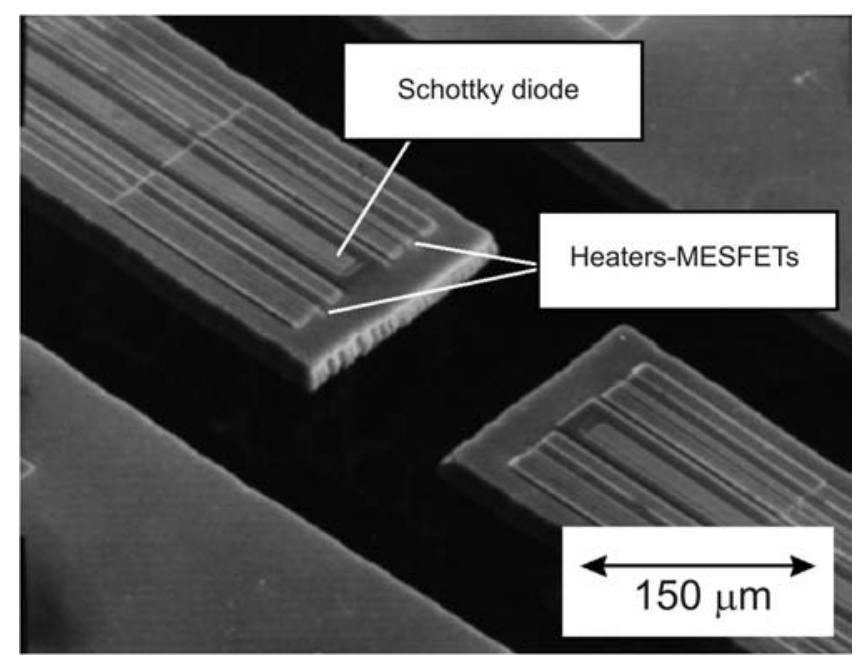

FIGURE 17. Real view of fabricated cantilever based power sensor [3].

described in [3]. GaAs MESFET technology combined with the micromachining technology of GaAs cantilevers was used to obtain a MEMS device for highly sensitive and broad frequency range power measurements.

A real view of the fabricated power sensor is shown in Fig. 17. It consisted of two MESFETs (as heaters) and a symmetrically placed Schottky diode (as a temperature sensor) monolithically integrated on $8 \mu \mathrm{m}$ thick GaAs cantilevers. A back side bulk GaAs micromachining technology described in Fig. 8 was used to fabricate the power sensor.

The principle of the measuring method is to balance the unknown power dissipated by one heater at a known power on the second one while maintaining a constant sensor temperature (about $50-100^{\circ} \mathrm{C}$ ) sensed by the Schottky diode. Controlling the current under constant voltage or the voltage under constant current on the second heater, a power/current or power/voltage converter, respectively, is obtained. The power sensor exhibited a sensitivity over $10 \mathrm{~V} / \mathrm{W}$ and yielded thermal resistance that exceeded $5200 \mathrm{~K} / \mathrm{W}$. The thermal time constant of $5 \mathrm{~ms}$ was measured using both electrical and optical methods [49, 50].

A new MMIC compatible sensor that measures RF and microwave power transmitted over a $50 \Omega$ coplanar waveguide (CPW) was presented [15]. A schematic cross-section through the power sensor is shown in Fig. 18. It consists of CPW that feeds the RF power to the sensor and an $\mathrm{AlGaAs}$ based thermopile that is proposed to measure the temperature increase. Both devices are integrated on a thin $\mathrm{AlGaAs}$ membrane. Back side bulk GaAs micromachining based on spray etching with $\mathrm{NH}_{3} \mathrm{OH} / \mathrm{H}_{2} \mathrm{O}_{2}$ solution was used to form a 1 $\mu \mathrm{m}$ thick undoped $\mathrm{Al}_{0.48} \mathrm{Ga}_{0.52} \mathrm{As}$ membrane. The etching technique has the advantage of high selectivity against $\mathrm{Al}_{0.48} \mathrm{Ga}_{0.52} \mathrm{As}$ while etching GaAs isotropically.

The sensor principle is based on the conversion of electrical power into heat resulting in a local temperature increase. Conversion is achieved via ohmic losses of the central conductor of the CPW, which is an intrinsic effect of the CPW and hence should not affect its performance. The heat losses are converted into measurable temperature differences by isolating the measurement region thermally. The central conductor is guided across 


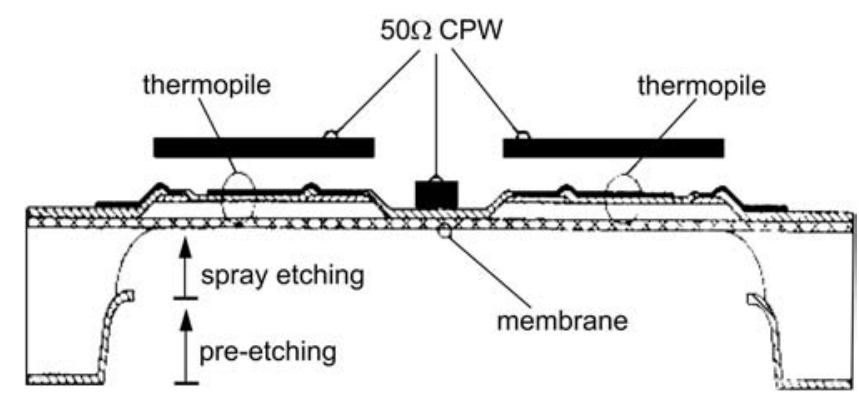

FIGURE 18. Schematic cross section through micromachined power sensor [15].

the AlGaAs membrane of high thermal resistance while the mass conductors cross the membrane in a bridge configuration with an air gap of $3 \mu \mathrm{m}$ to prevent undesirable heat losses. Detection of the temperature difference between the central conductor and the rim of the chip that presents the heat sink is realized with 24 series-connected $\mathrm{AlGaAs} / \mathrm{CrAu}$ thermocouples. Sensitivity of this power sensor of $1.1 \mathrm{~V} / \mathrm{W}$ has been achieved with a reflection coefficient of 0.1 at $10 \mathrm{GHz}$.

A further improvement of the power sensor microwave performance has been achieved using CPW designed to have a terminating load configuration [16]. Schematic diagram of the sensor is shown in Fig. 19. CPW is designed to have a characteristic impedance of $50 \Omega$. It is terminated with a resistive load that is matched to the line impedance. To achieve improved high frequency performance, two $100 \Omega \mathrm{NiCr}$ thin film resistors are connected in parallel between the centre conductor and the ground metallization. This load absorbs

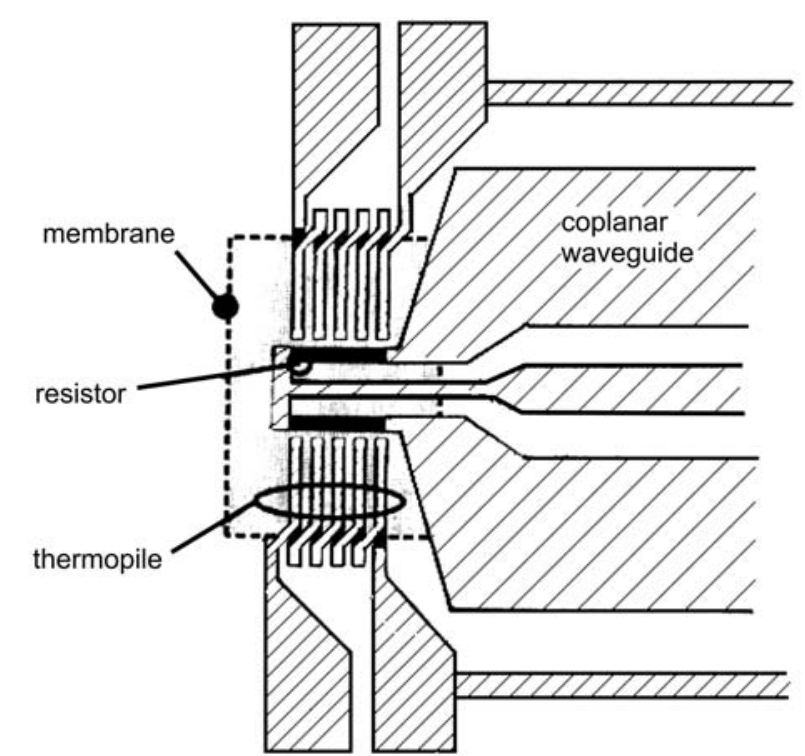

FIGURE 19. Schematic diagram of sensor with CPW terminated by two $100 \Omega$ thin film resistors in parallel [16]. 


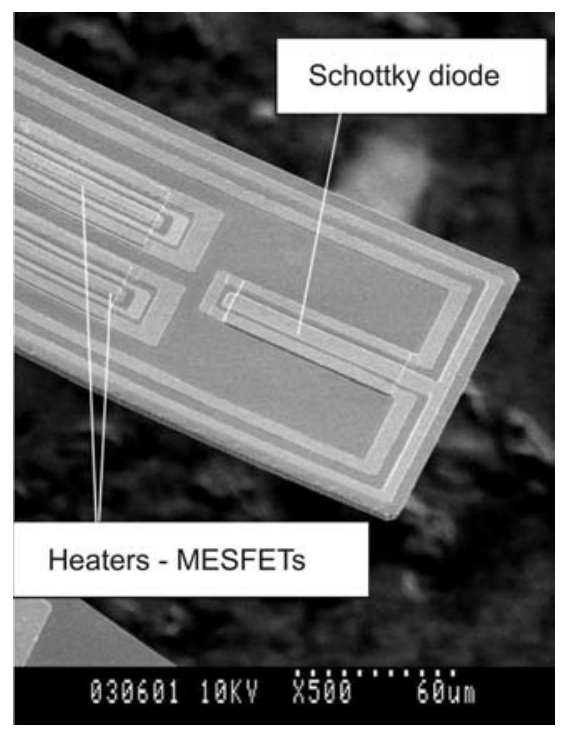

FIGURE 20. Front side view of $2 \mu \mathrm{m}$ thick cantilever of power sensor [14].

the microwave power and converts it into heat. The power sensor of this design concept exhibited sensitivity of $2.02 \mathrm{~V} / \mathrm{W}$, inherent linearity and a thermal time constant $0.5 \mathrm{~ms}$.

Further improvements in the performance of the power sensors of the cantilever based design concept as shown in Fig. 17 have also been demonstrated [7, 14, 22, 23, 51]. To improve the electro-thermal conversion efficiency of the sensor, cantilevers with thickness $2 \mu \mathrm{m}$ were used. A high resistive low-temperature grown GaAs layer (LT-GaAs) was designed to define the thickness of the cantilevers in order to suppress the parasitic leakage currents in the Schottky diode temperature sensor. The front side view of the sensor cantilever is in Fig. 20. Comprehensive thermo-mechanical characterization of the sensor was carried out [14, $22,51]$. As expected, the electro-thermal conversion efficiency of the sensor was improved substantially. It can be demonstrated by a direct measurement of the power-to-temperature $(P-T)$ conversion characteristics at different ambient atmospheres (Fig. 21). The corresponding thermal resistance values were determined to be $14000 \mathrm{~K} / \mathrm{W}, 17000 \mathrm{~K} / \mathrm{W}$ and 31000 $\mathrm{K} / \mathrm{W}$ for air, argon and vacuum environments, respectively. This is consistent with the decrease of the thermal conductivity of these gaseous media. The increased sensitivity of the sensor cantilever to the thermal conductance changes of the ambient gaseous environments could also be useful for the design of GaAs micromachined vacuum sensors. Moreover, the sensor cantilever exhibited a significant deflection induced by the different thermal expansions of the GaAs cantilever layer and of the top device interconnecting metallic layers (Ti/Au), thus by the so-called bimetallic effect. The cantilever deflection was changed by the power dissipated in the heaters (cantilever temperature increase). This effect can be used for the design of thermally actuated micromachined actuators [22, 23, 52]. Finally, the temperature time constant of $2.79 \mathrm{~ms}$ was obtained from both experiment and simulation.

A further progress in the design of micromachined power sensors has been demonstrated by implementation of pHEMT processing technology compatible with the micromachining technology of the micromechanical structures (membrane, bridge, cantilever) [1, 9, 39-42]. 


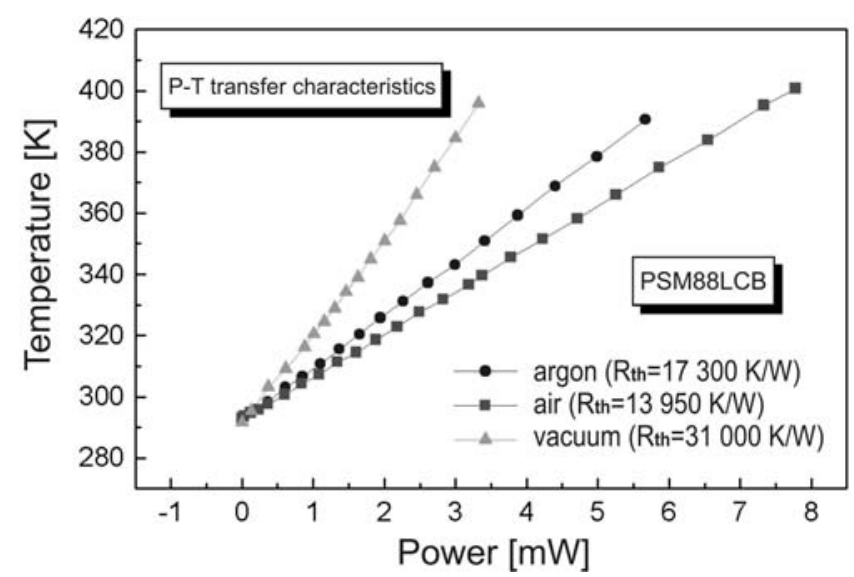

FIGURE 21. Power-to-temperature $(P-T)$ conversion characteristics measured at different ambient atmospheres [22].

A schematic cross-section through polyimide-fixed micromechanical structures to be integrated with the pHEMT as a heater is shown in Fig. 22a. The technology starts with MBE or MOCVD growth of GaAs heterostructures on a semi-insulating substrate (SI-GaAs) (Fig. 22b). The layer system represents the pHEMT design. A silicon deltadoped layer is formed in the $\mathrm{Al}_{0.22} \mathrm{Ga}_{0.78} \mathrm{As}$ barrier layer, and it is separated by a $3 \mathrm{~nm}$ thick undoped $\mathrm{Al}_{0.22} \mathrm{Ga}_{0.78} \mathrm{As}$ spacer layer from the $\mathrm{In}_{0.2} \mathrm{Ga}_{0.8} \mathrm{As}$ channel. $\mathrm{A} \mathrm{GaAs} / \mathrm{Al}_{0.3} \mathrm{Ga}_{0.7} \mathrm{As}$ $(700 / 300 \mathrm{~nm})$ heterostructure buffer layer under the channel was designed to define the thickness of the cantilever or bridge structure. Based on the bulk GaAs micromachining technology as described in Fig. 9, MTC devices were fabricated to study the electro-thermal properties of polyimide-fixed cantilever and bridge structures. To fabricate the MTC devices, front side surface processing and micromachining are combined with back side bulk GaAs micromachining. Basically, the process flow is divided into two steps involving front side processing of pHEMT structures followed by surface micromachining of the cantilever

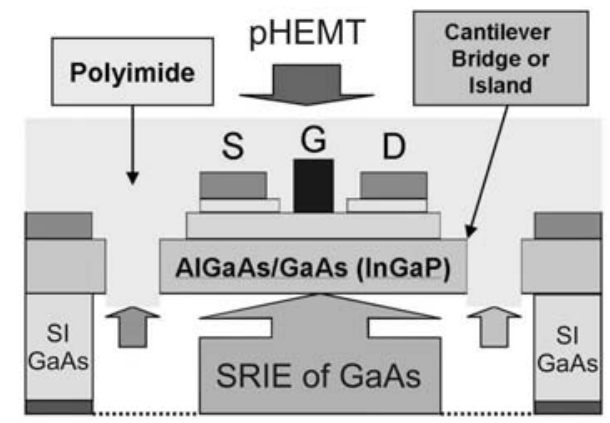

(a)

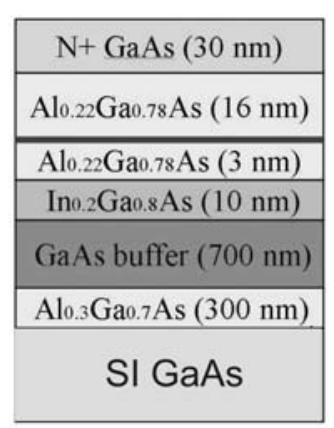

(b)

FIGURE 22. Polyimide-fixed micromachined thermal converter, (a) schematic cross section through polyimidefixed micromechanical structures, (b) pHEMT heterostructure layer design. 


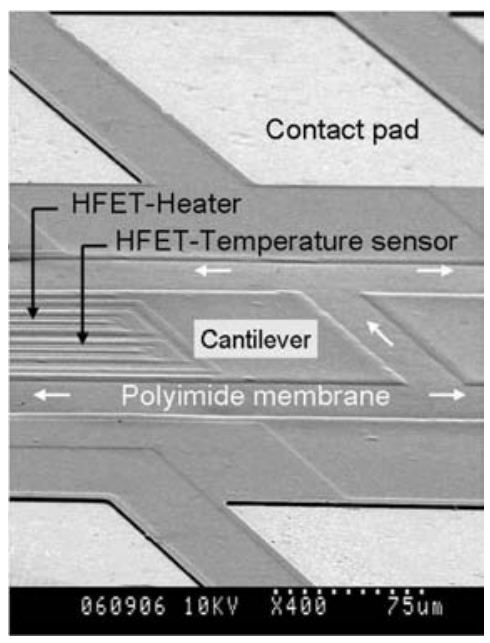

FIGURE 23. Front side view of polyimide-fixed cantilever based MTC device [41].

or bridge structures, and back side bulk micromachining of the GaAs substrate as the last processing step. Fig. 23 shows the real front side view of the cantilever based MTC device. It consists of two pHEMTs monolithically integrated on a cantilever micromechanical structure fixed by a polyimide membrane. In this integrated approach one of the pHEMT is designed to serve as a heater and the second one is used for temperature sensing.

A pHEMT's Schottky gate diode is proposed to sense the temperature of the micromechanical structure. The standard metallic leads ( $\mathrm{Ti} / \mathrm{Au}$ ) patterned on the top of the cantilever were used to connect the pHEMT active areas (Source, Drain and Gate) with the contact pads outside the micromechanical part of the device.

The electro-thermal conversion efficiency of the fabricated MTC devices was investigated. Fig. 24 shows the measured power to diode voltage $(P-U)$ conversion characteristics of the cantilever and bridge based MTC devices. Excellent linearity in the conversion is obtained for both devices with sensitivities as high as $-15.6 \mathrm{~V} / \mathrm{W}$ and $-14.3 \mathrm{~V} / \mathrm{W}$, respectively. The relevant thermal resistance values were found to be $13600 \mathrm{~K} / \mathrm{W}$ and $10400 \mathrm{~K} / \mathrm{W}$ for the cantilever and bridge based MTC devices, respectively.

Recently, a novel approach in design of MTC device has been introduced [53, 54]. It is based on so called a suspended island structure. Thin polyimide membrane with a low mechanical stress and thermal conductivity is used there for mechanical fixation and thermal isolation of the GaAs/AlGaAs island structure (see Fig. 9). This micromachining approach permits to fabricate the micromechanical structures of high thermal resistance values and negligible deformation (see bellow). It also permits the structures to be fully thermally isolated against the ambient atmosphere, so the influence of the ambient thermal conductivity changes can be considered to be negligible. The island based MTC creates a heart of a microwave transmitted power sensors (MTPS) [55] that should be capable to sense the transmitted power given by the product of the electromagnetic field vector components $\left(\mathrm{P}=\frac{1}{2} \operatorname{Re}\left[\int_{\mathrm{S}} \mathrm{E}_{\mathrm{t}} \times \mathrm{H}_{\mathrm{t}} \cdot \mathrm{z}_{\mathrm{o}} \mathrm{dS}\right]\right)$.

Fig. 25 shows a schematic cross-section through the island based MTC device. It consists of the GaAs pHEMT as a microwave heater and thin film meander-like resistor 


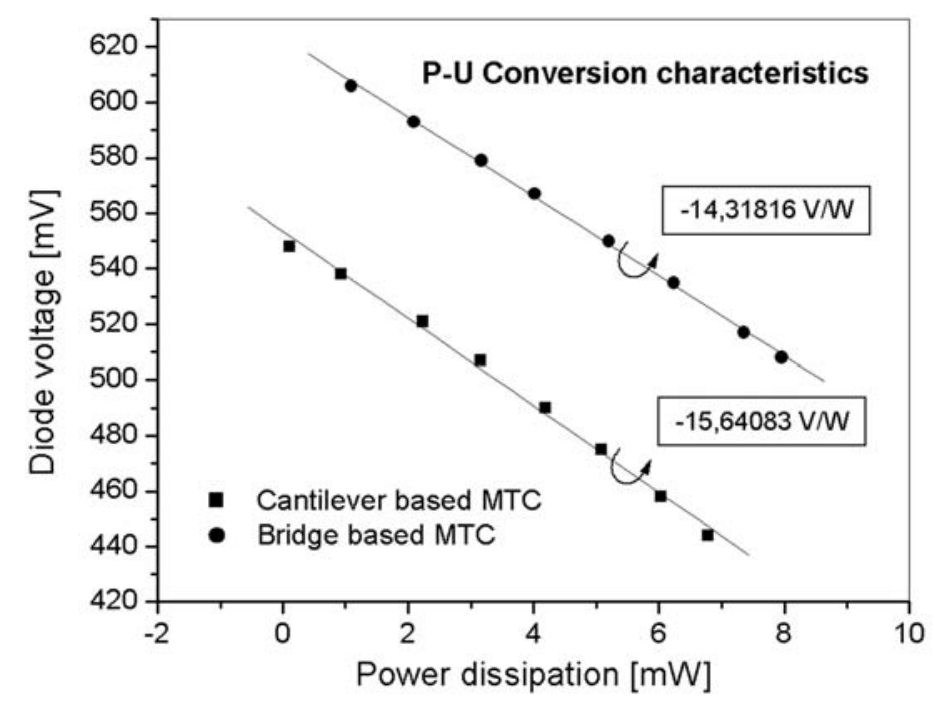

FIGURE 24. Measured power to diode voltage $(P-U)$ conversion characteristics of both cantilever and bridge based MTC devices.

as a temperature sensor. The both devices introduced are monolithically integrated on $1 \mu \mathrm{m}$ thick GaAs/AlGaAs island structure fixed by $1 \mu \mathrm{m}$ thick polyimide membrane. The standard metallic leads $\mathrm{Ti}(50 \mathrm{~nm}) / \mathrm{Au}(150 \mathrm{~nm})$ placed on thin suspended GaAs crossbridges of the island structure were used for interconnection of the pHEMT and temperature sensor with the sensor controlled circuit realized in monolithic integration on the bulk GaAs substrate [55]. AlGaAs/InGaAs/GaAs heterostructure layer system as shown in Fig. $22 \mathrm{~b}$ was designed to be used for both pHEMT technology and suspended island structure fabrication. Details of the fabrication process are in principle the same as for polyimide fixed cantilever based MTC device (Fig. 23). A real view of fabricated MTC device is shown in Fig. 26.

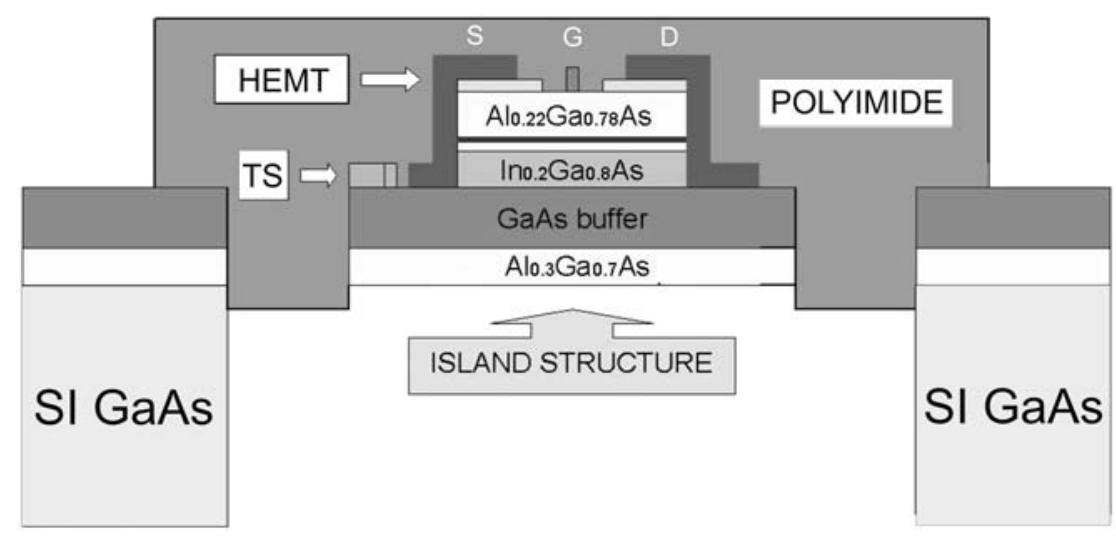

FIGURE 25. Schematic cross-section through polyimide-fixed island based MTC device. 


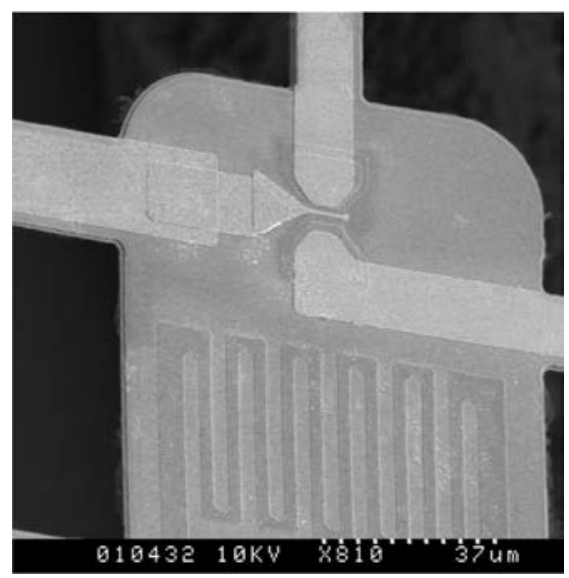

FIGURE 26. Real view of fabricated island based MTC device [53].

An electro-thermal [53] and thermo-mechanical [54] analysis of the MEMS device has been carried out. The both power to temperature $(\mathrm{P}-\mathrm{T})$ and power to voltage $(\mathrm{P}-\mathrm{V})$ conversion characteristics were determined. An excellent linearity in the electro-thermal conversion was observed. The preliminary experimental results obtained show the thermal resistance value as high as $9650 \mathrm{~K} / \mathrm{W}$ and device sensitivity of $9.74 \mathrm{~V} / \mathrm{W}$. The micromachined concept of the island based MTC device had no substantial influence on both room-temperature and low-temperature performance of the integrated $0.5 \mu \mathrm{m}$ gate length pHEMT heater. Moreover, the dynamic behavior of the device was investigated using two different methods. The thermal time constant about $1.32 \mathrm{~ms}$ was determined from the measured time response of the temperature sensor voltage and deformation, respectively.

As it was shown, the heterostructure based design of MTC devices is often very complicated. It contains various materials of each other different thermo-mechanical properties. To study the thermally induced thermo-mechanical effects and phenomena in the MTC devices of the multiplayer basis on the required micro- and nanoscale a novel methodical approaches are strongly desired. Therefore, in the next part of this chapter non-conventional contact-less optical methods are introduced to analyze the basic thermo-mechanical properties of the selected GaAs thermally based MEMS devices. They permit (in situ) to study the device 3D nano-deformations induced by the temperature changes in both stationary and non-stationary dynamic process conditions.

\section{MEMS Device Thermo-Mechanical Characterization}

\section{SURFACE 3-D PROFILING}

The use of laser based and other applied optics tools for inspection and diagnostics of MEMS is predetermined by their noninvasive and contactless nature. One of the applications where the optics can be widely utilized is the surface relief profiling and also surface roughness inspection. Such a deformation analysis of MEMS components provides 
information about technology imperfections, shape deviations formed at the depositing and etching processes and about the deformation induced by residual mechanical stresses. It is increasingly important to measure surface parameters such as coplanarity, warpage. heigh steps and surface roughness of different parts on MEMS structure. The shape monitoring is of prime importance particularly for components based on membrane-like design which is in MEMS technology frequently the case.

Nowadays, several methods can be arranged and adapted to such a purpose. As a rule, the applications require non-contact measuring techniques with a sensitivity in height variations at least bellow $0.1 \mu \mathrm{m}$. The techniques that fulfill this requirements include variety of modifications of convenient interferometry, white light interference microscopy and confocal microscopy.

Dealing with specular surfaces in order to characterize 3-D shape of small MEMS structures and components, mostly the interference principle is taking into account. Using the coherent or partly coherent light, the interference between light reflected from the surface and that returned back from a reference flat produces fringes. The resulting fringe pattern is a contour map of the phase differences between the two wavefronts. Provided that the two waves have equal intensity $I_{0}$ the intensity distribution of interference pattern is described by the well known expression

$$
I(x, y)=2 I_{0}[1+\cos \delta(x, y)]=2 I_{0}\left[1+\cos \frac{2 \pi}{\Lambda} \Delta z(x, y)\right]
$$

where $\delta(x, y)$ is the phase difference between two wavefronts at a given point $(x, y), \Delta z(x, y)$ is the optical path difference and $\Lambda$ is the wavelength of light. The result of interference is a set of "fringes" which represent contours of the object surface with a fringe spacing of $\Lambda$

$$
\Delta z(x, y)=N \frac{\Lambda}{2}
$$

where $N$ is an interference order of fringes. At the observation the object image is overlapped by a system of interference fringes which match the contours of vertical relief of the surface in view of reference plane. The ratio of vertical/horizontal resolving power reaches usually more than tens, but if the fringes are sharpened by means of multiple-beam interferometry or by image processing intensity interpolation between fringes spacings, it is possible to achieve the vertical resolving power up to nanometers scale. Laser interferometry is probably one of the most commonly techniques used for MEMS surface profiling.

In microinterferometry, there are three basic optical schemes-Michelson, Mirau and Tolansky interferometers, depending on the position of reference glass flat in the arrangement. Figure 27 shows the interferometers applicable for the measurement of small scale surface areas of MEMS. Each of these types of geometry has its own specific features. Michelson type of interferometer (Fig. 27a) is e.g. regularly used in connection with microscopic objectives of smaller magnification $1 \times$ to $5 \times$, larger viewing field and also larger working distance. An example of deformation contours on the GaAs strip with metallic connections is shown in Fig. 28. If higher magnification of image is needed the measurement can be carried out by Mirau microscopic objectives (Fig. 27b). Both the Michelson and the Mirau types of microscopic interferometers are currently commercially available as special microscopic accessories. 


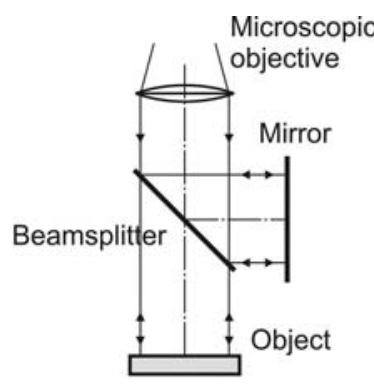

(a)

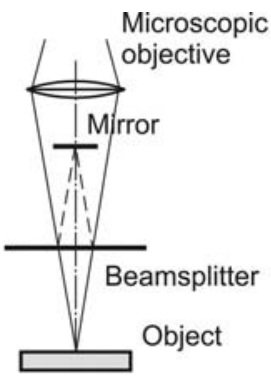

(b)

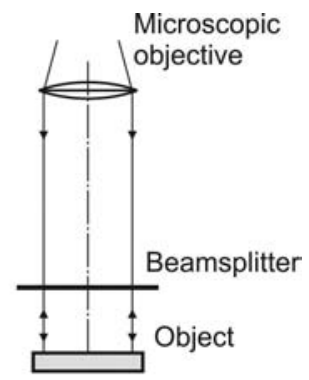

(c)

FIGURE 27. Types of interference microscope based on, (a) Michelson, (b) Mirau, and (c) Tolansky scheme.

When the coherence light source can be used, which denotes the CW laser or laser diode with sufficient coherent length, Tolansky interferometrical layout (see Fig. 27c) can be arranged. This type of interferometer has been developed as a universal testing device also in our laboratory (see Fig. 29). Its basic element is the polarizing beamsplitter cube consisting of two prisms. The interference effect is generated in the air gap between the specimen surface and the lower flat of cube. In the interferometer the optimal intensity conditions are adjusted by rotating of plane of polarisation. By this way also disturbing secondary reflections are minimised and the interference pattern is not disturbed by parasitic fringes. As a rule, the intensity of light reflected from the flat reference glass surface not covered by a reflection coating is adequate to create contrast interference pattern. The splitting cube is fixed on a two-axes adjustable holder. Besides the good flatness of the cube surfaces, the only critical element is aberration free collimator objective. The image of object is viewed and magnified at a suitable measure by microscopic objective and built-in CCD camera. Regarding finite size of the beamsplitter cube a microobjective with long working distance has to be used. Large field of view, easy adaptation of the arrangement to variety

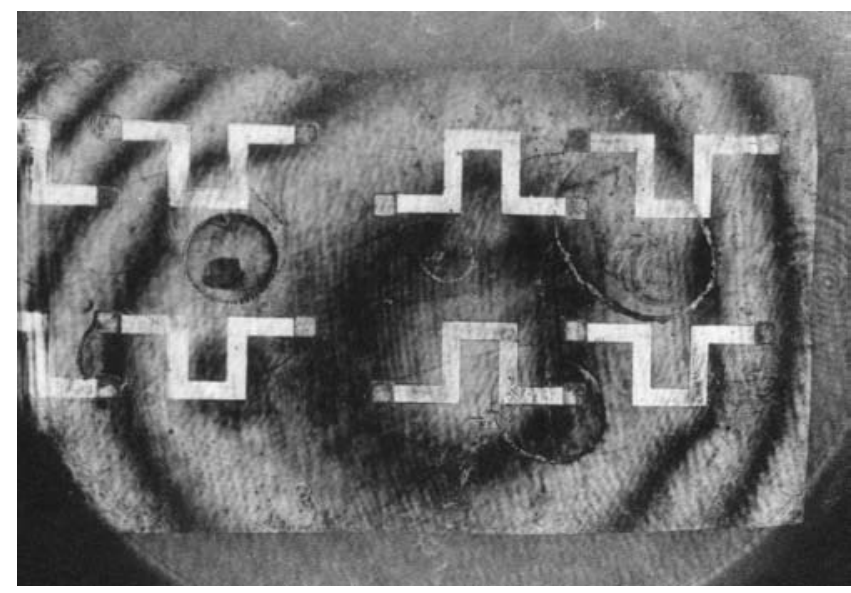

FIGURE 28. Interference contours on a strip element of MEMS. 
TIBOR LALINSKÝ ET AL.

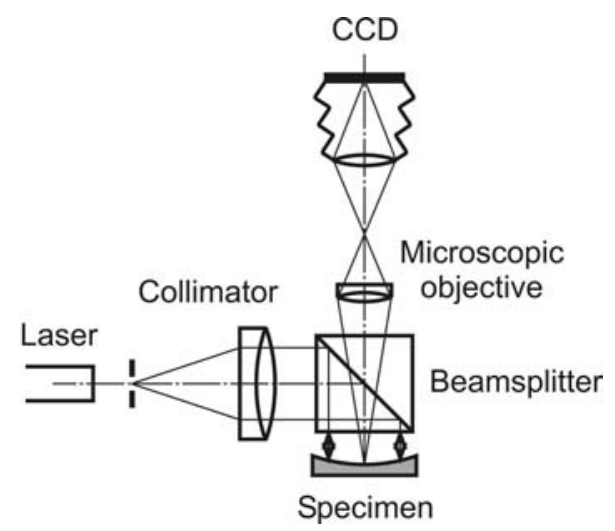

FIGURE 29. Microinterferometrical setup based on Tolansky optical scheme.

of purposes and also the possibility of simultaneous observation of thin film interference pattern on transparent surface covering are the practical advantages of the device.

Several kinds of components made of GaAs based technology of membrane-like structures has been tested using this tool [14, 19, 25, 41, 42]. In Fig. 30 the interferometrically measured profiles along the length of free cantilever are drawn. As it can be seen, besides the visualisation of steady-state profile of the cantilever after its technological forming, the changes generated as a thermal response by acting feeding power has been inspected, too.

Another alternative of Tolansky arrangement is the reference flat positioned by laying it on the measured surface or in its vicinity. If this flat is kept at a small angle to the surface mean level then the set of profiles is created. In this case the interpretation of fringe pattern is not so clear and straightforward comparing the height contour fringes presented by previous schemes, nevertheless, the shape of surface can be computed too, from the data of fringe pattern viewed by a CCD camera. One of the advantages of this adjustment is the possibility

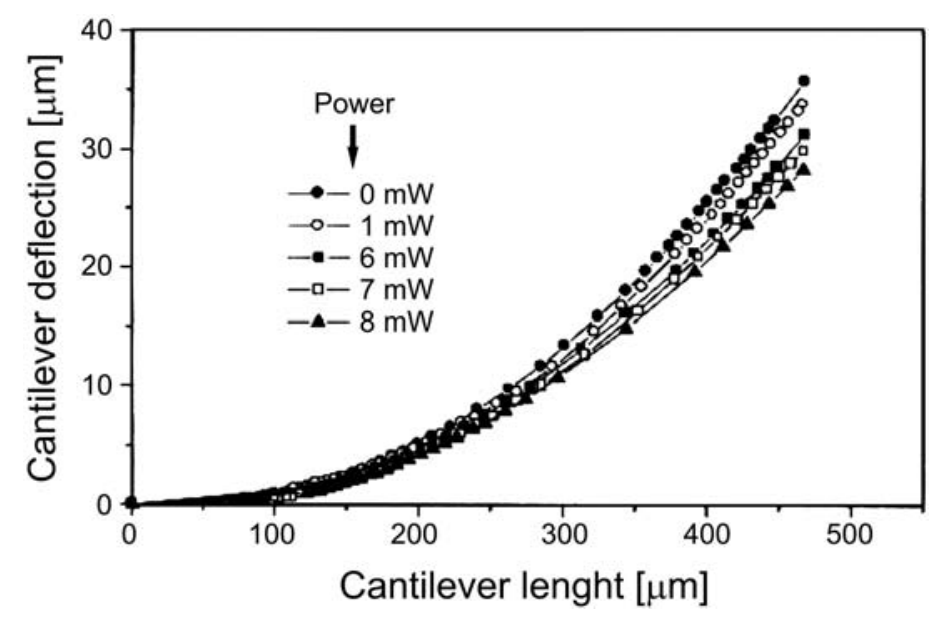

FIGURE 30. Steady-state profiles of cantilever deflection as a function of power dissipation [25]. 
of simple varying of sensitivity in line-of-sight direction. This interferometry is especially usefull when the surface defects diagnostics is necessary.

In addition to surface profiling, the basic scheme shown in Fig. 27c can be simply adapted to detect the surface layers thickness variations. In the design of MEMS, there is a number of layers transparent in visible light or in near infrared (NIR) region. The advantage of this transparency can be taken to create thin layer interference fringes and thus to inspect the area distribution of layer thickness [56, 57]. However, in experimental practice, the origin of fringes of equal thickness depends on appropriate combination of indices of refraction at the mutual interfaces air-layer as well as air-substrate. On the other hand, thin film interferency often provides information about defects, homogenity and possible stress concentrations inside the layer. Remarkable chance of thin film interferometry follows also for the inspection of small but identified disturbances of fringes during transient thermal event or change in steady state temperature distribution. Visible changes in thin film interference pattern are caused by optical phenomenon of index of refraction changes with the changes in temperature of transparent material. Knowing this functional dependence (see e.g. $[58,59])$, the area temperature distribution on the surface considered can be mapped. Detecting of temperature development on GaAs based beam element thermally isolated by surface polyimid coating is presented in $[49,50]$ where the temperature variations with a sensitivity of about $1.0 \mathrm{~K}^{-1}$ were successfully identified.

On the other hand, observing thin film fringe pattern, experimentalist is frequently confronted with the problem of disturbancy of the surface profile interference pattern by fringes coming from thin layer interferency. A simple solution how to separate two families of fringes is the use of low coherence light source. Also the deterioration of temporally coherent laser light can be carried out. To do it, the ground screen diffuser is putted into the beam illuminating object, then the light with coherence length smaller than the path difference of reference vs. object beams completely cancels the fringes of profile contours (see Fig. 31a).

An alternative way how to obtain contrast thin layer interference visualising thickness/index of refraction variations is the area scanning by laser confocal microscopy.

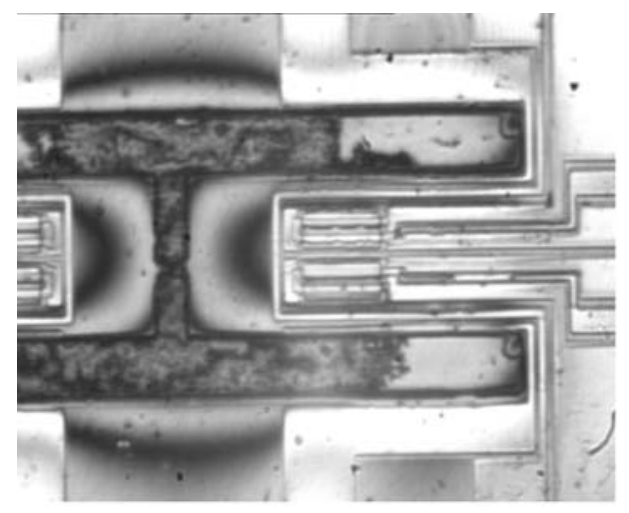

(a)

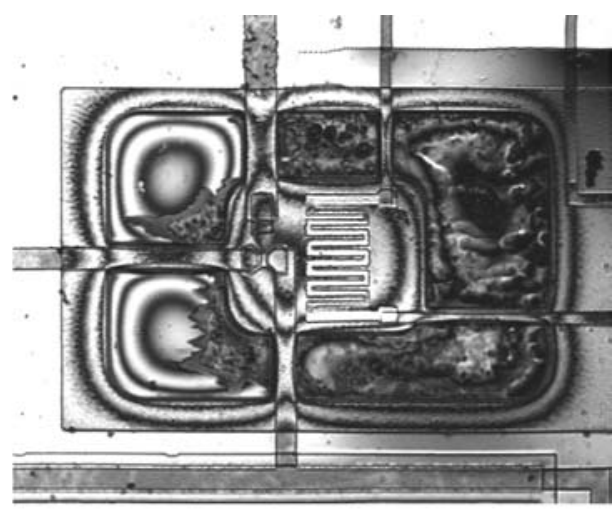

(b)

FIGURE 31. Thin film interference fringes obtained, (a) by laser diode $532 \mathrm{~nm}$, and (b) by Zeiss LSM 510 META confocal microscope scanning using $633 \mathrm{~nm} \mathrm{CW} \mathrm{He-Ne} \mathrm{laser.}$ 
In Fig. 31b thin layer interferency is illustrated on surface polyimid film with the thickness of about $1 \mu \mathrm{m}$. By this polyimid the membrane structure of GaAs/AlGaAs island element, slits between the island and the bulk within the area of clearly visible boundaries are covered, and the reflection from back and front side and subsequent interference of confocal microscope probing light creates the thin film interference fringes. Mechanism of their forming follows from confocal microscope SW processing procedures where pixel-to-pixel depth intensity profiles are processed for searching the best maximum intensity positions, thus visualising the localization of both destructive and constructive interferency. As seen, the fringes visualises also defects caused by imperfections of etching process and polyimid layer depositing. The layer thickness distribution reflects the influence of capillarity and/or shrinkage effects at edges and corners.

Low coherence or white light interference microscope [60-63] is an advanced tool with some specific advantages over the "conventional" interferometric technique [60, 64]. It is primarily the ability to strongly reject light that has undergone scattering outside which gives the generation of speckles when illuminating by coherent light. When a low coherence light is used in interference microscope, and the microscope objective is moved continously in line-of-sight axis, the contrast of interference fringes is modulated depending upon the optical path difference. A low coherence interferogram can be described by a constant mean intensity $I_{0}$ and a series of sinusoidal fringes modulated by envelope function [60]

$$
I(x, y)=I_{0}(x, y)[1+V(x, y, \Phi) \cos \Phi]
$$

where the function $V(x, y, \Phi)$ is the visibility or fringe contrast, which varies much more slowly with optical path difference than the fringe phase $\Phi=\Phi(x, y)$. A basic principle is the searching for the position of maximum contrast simultaneously for an array of image points. Thus, a 3-D surface profile can be measured by finding the maximum peak position of the fringes modulation in a CCD camera. At present, there have been many approaches of calculation algorithms to find this maximum as reliably and precisely as possible and in a shortest time. Modern white light interference microscopes are typically equipped with Mirau objective and a moderately filtered white light source is halogen lamp or high brightness LED. The interference fringes envelope has a width of some micrometers, hence the accuracy of measurements can be defined as tens of nanometers. Last years the applications of white light interferometry to MEMS/MOEMS devices testing have become increasingly important.

Sometimes the interferometric technique cannot be applied for surface geometry profiling, for example, when the surface is optically rough and reflects the light diffusely. Problem solution in this case can be application of confocal microscopy [65-67]. Convenient confocal microscopy belongs to large group of optical techniques involving lateral mechanical scanning as part of the 3-D data acquisition. In optical scheme of confocal microscope, light of point-like source is projected into the object focal plane of microscope objective. The light reflected backward is collected by the same objective and after passing through the beamsplitter it is projected onto a detector pinhole. Only the focused positions of the sample surface give the maximum signals and light is strongly reduced by pinhole when the surface is in defocused position. In this manner the plane of best focusing is defined and three coordinates location of measuring point is recognised. The lateral resolution of 3-D profiling is related to the smallest spot diameter $d$ that follows from the Airy disc 


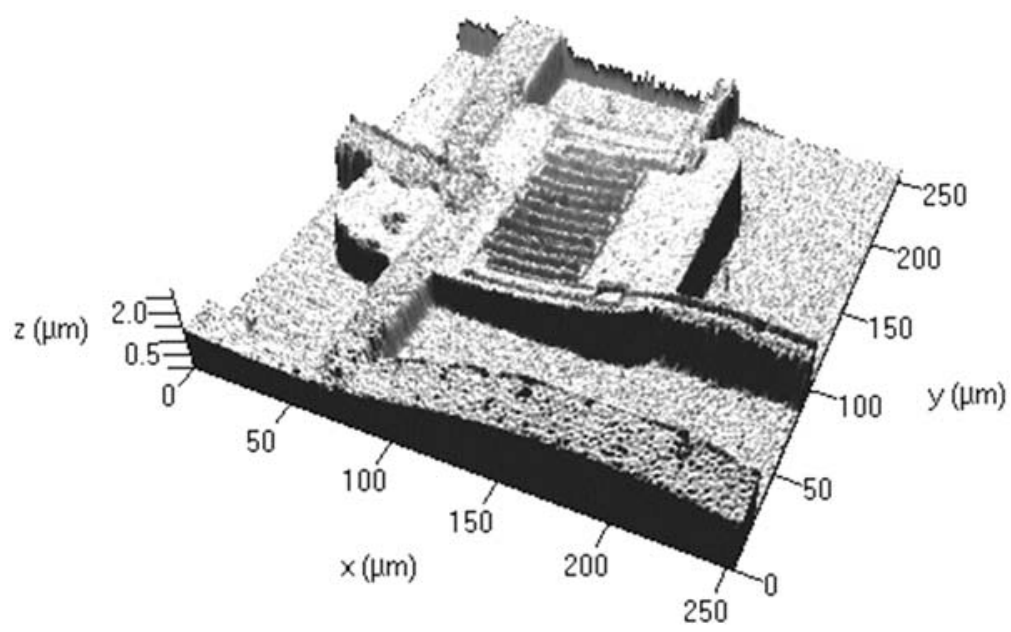

FIGURE 32. 3-D landscape view on GaAs/AlGaAs based MEMS micro-island element obtained by Zeiss LSM 510 META confocal microscope.

expression

$$
d=1.22 \frac{\Lambda f}{D}=1.22 \Lambda N . A
$$

where $f$ is the objective focal distance $D$ is its aperture diameter and $N$.A. is the numerical aperture of the microscope objective. The best focusing is obtained with high numerical apertures, where in fact, the maximum value of $N . A .=0.6 \div 0.8$ can be taken into account which leads to the spot diameter in a range of $1 \div 2 \mu \mathrm{m}$. Vertical accuracy of focusing depends on photoelectric detector noise properties, furthemore on the measure of searching focus algorithm sophistication, but also on the properties and complexity of measured surface. 3-D landscapes/height profiles illustrated in Fig. 32 and Fig. 33 were obtained on Zeiss LSM 510 META multiple wavelengths laser confocal apparatus. The quality (specularity and microsurface inclinations) of all the parts of the membrane GaAs/AlGaAs

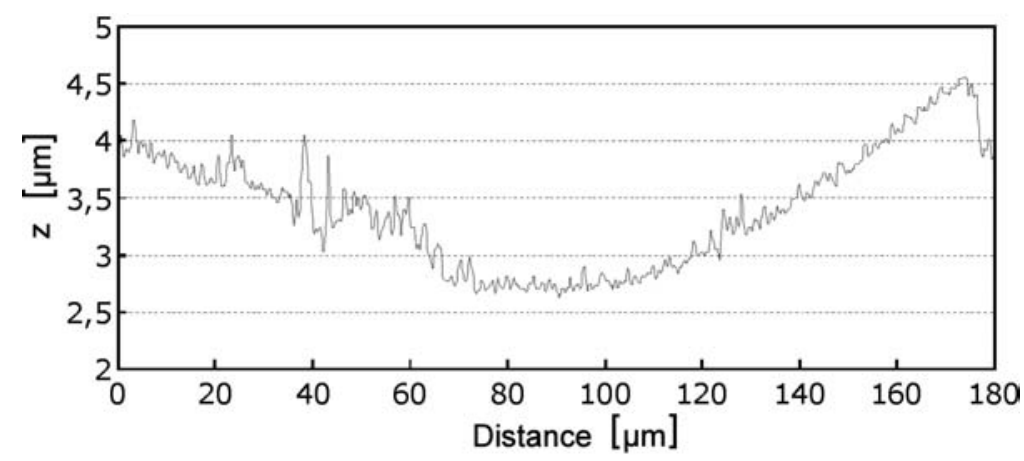

FIGURE 33. One of profiles of multilayers membrane micro-island element warped by internal residual stresses. 
microisland MEMS surface has not been uniform and scanning of the MEMS device required retrieving of optimal working wavelength of light, appropriate algorithm of focal position detecting and also the choise of suitable parameters of microobjectives used. As it was noticed above, for some specimens covered with polyimid, the thin layer interferency has appeared and excluded proper interpretation of primary recorded data of depth intensity profiles. In this case the problem has been eliminated by processing and following averaging of the data acquired by using of different light wavelengths. As it can be assessed also from the one of the cross-section profiles at the real mirror-like parts, the standard deviation $\sigma$ is less than $0.1 \mu \mathrm{m}$, the value frequently cited for confocal microscopy.

\section{DETERMINATION OF GaAs/AlGaAs MATERIAL PARAMETERS}

At the designing process of the MEMS device the knowledge of structural mechanical stress state is often essential to the right weighting of all the mechanical proposal aspects. Nowadays, the development and fabrication of MEMS devices especially based on GaAs technology, has not been realized using only conventional well established procedures and known material parameters. Increasing design and performance demands in the near future will require more exact and complete information considering both the mechanical and thermo-mechanical materials properties and their mutual interactions in multilayer system. It is well known that most of convenient mechanical test techniques and procedures were developed to bulk materials and are not generally suitable for thin film applications. Therefore, new or modified methods has to be elaborated.

The basic requirement of any test used to gather information about materials parameters is an application of controllable externally applied loading and measurement of deformation response (static or dynamic) of mechanical components to this actuation. It can be noted that the most widely used techniques of deformation measurements on microcomponents are the methods where the applied optics principles are employed. The techniques can be applied such as interferometry, electronic speckle pattern interferometry (ESPI), point-like laser reflectance measurement or the measurement by autocollimation arrangement where the light reflected from the object is analysed in or nearly back focal plane of imaging lens. Besides more or less convenient microtensile testing, when the CCD camera or ESPI are used for deformation tracking, a free-standing thin film beam is bended to plot material loading diagram and subsequent elastic moduli extraction. Regularly used vibrational (or resonant) testing is a variation of the latter method.

Another widely used procedure of Young's modulus and membrane tension stress measurement is the method usually called as bulging test. In this case, the membrane component is deformed by applying one-side overpressure. Provided that $t$ is the thickness of the membrane, $a$ is the lateral size and $w$ is the central deflection of bulged membrane induced by overpressure $p$, it can be writen [68]

$$
p(w)=K_{1} \frac{\sigma t}{a^{2}} w+K_{2}(v) \frac{E t}{a^{4}} w^{3}
$$

where $E$ and $\sigma$ are the elastic modulus and the membrane tension stress, respectively. The constants $K_{1}, K_{2}$ depend only on the lateral geometry of membrane component. Optical 
detection of current value $w$ can be effectively accomplished by the whole viewing field observation, interferometrically or by autocollimation searching shifted focus.

\section{RESIDUAL STRESS ANALYSIS}

The presence of residual stresses is a characteristic feature of almost all the multilayered structures composed of different materials. The residual stress result from heterogenity of material properties and from treatment by thermal but also by mechanical means. The last group can be often identified e.g. after grinding and finishing a mirror-like $\mathrm{GaAs}$ (or $\mathrm{Si}$ ) wafer front side area. The mechanical intervency (or chemical when chemical polishing is applied) affects upper layers of the single crystalline material whose microstructure tends to follow predominantly its crystallographic structure. This phenomenon is visible on almost all the maiden polished surfaces of wafers at the inspection of initial wafer deformation. The surface flatness curving either large-scale or only negligible, shows ellipsoidal shape, and sometimes even saddle-like warping, with the main axes of symmetry in accordance with orthogonal axes of monocrystal.

However, in fabrication processes the main role play the residual stress state the origin of what is the different thermal dilatability of coating and substrate and intrinsic mutual interaction, both inherent practically in all of the deposition processes. As it is known, the origin and nature of internal residual stresses are the sources of many mechanical effects in coatings and repeatedly are of prime concern when dealing with multilayer complicated system.

Experimental detection and evaluation of residual stresses can be divided into two categories:

i/ measurement of steady state deformation induced by residual stress in free-standing structural elements

ii/ measurement of deformation forced to clamped thin plate or membrane-like elements by controllable loading

The well known measurement of film stresses of coated wafers belongs into the former category where the coating film stress is determined by measurement of wafer thin plate free deformation. The method is appropriate if interaction between film and substrate is necessary to know and the conditions of depositing process can be simulated, identical with that used at actual fabrication of designed MEMS device. Assuming the homogeneous stress distribution throughout the wafer area, the relationship between the searched film stress and the induced deformation is very simple, described by Stoney formula (see bellow). The only one parameter which has to be determined in this case, is the radius of curvature of spherically bulged substrate.

In principle, this small spherical deformation of the wafer surface can be accomplished by a number of optical methods. The presence of the specular surface makes the task relatively simple to solve from the point of view of optical techniques, testing arrangement can be materialised by classic means. In a market, several measurement systems commercially available are intended to such a purposes $[69,70]$. The systems of KLA Tencor are especially adapted to the thermal stress evaluation and are even equipped with a thermally controlled testing chamber. 
In spite of that, the automated systems are not always matched with the varying requirements of designing researcher. Therefore, the comparative analysis has been performed of several optical techniques under consideration [71,72]. Apart from the using of convenient interferometry, common drawback of which is frequently its even excessive sensitivity, and the need to use large precise optical elements, a holographic interferometry setup has been realised. One of the main attractions of holographic interferometry is the comparative principle, attribute that can be exploited to separate of initial flatness distortions. Such distortions many times are not spherically shaped and as it has to be noted, the value of deformation often is of the same order as that induced by thermal/intrinsic stresses. The advantage of holographic direct optical differential mode of measurement has proved particularly when the small deformation changes are needed to be determined. Practical drawbacks of holography, mainly its technical realization, has been overcome by installing of electronic speckle pattern interferometry (ESPI) with PC based image processing.

Another optical principle tested was a specific variant of classic Ronchi's ruling setup. In the method with no moving components and no large glass prisms and mirrors (with extention of large diameter abberation free doublet of objective lens) a coarse grid positioned at back focal plane is used. The rays reflected from the specular object are collected at the focal plane and are passing through the coarse $(1 \div 5$ lines per $\mathrm{mm})$ grid. The grid, in fact binary filter of optical filtration scheme, is projected onto the screen in a view of fringes visualising slope contours of the surface tested.

Among simple and unpretending optical scheme realisations, a method of autocollimation or searching focus, can also be named [73]. As it was mentioned above, the inspection of thin layer residual stresses on wafers requires only information about the radius of curvature of the approximatelly spherical surface. Similarly as in the previous technique of slope contours, the beam of parallel rays is reflected from polished surface (see Fig. 34). In the case of reflection from flat surface the light after passing backward through the long focal distance (about $1 \mathrm{~m}$ ) objective is concentrated precisely into focal plane. The change of reflected area to spherical shape (concave or convex) will shift the "focus" spot along optical axis out of initial position. The focus shift $\Delta$ is measured to determine the value of surface curvature $R$ related with the shift by a simple formula derived by elementary ray tracing laws

$$
R=2\left(\frac{f^{2}}{\Delta}+f-l\right)
$$

where $f$ is the focal distance of objective lens and $l$ is the mutual distance objectivespecimen. Generally, in the case of orthogonally symmetric surface which is on wafers

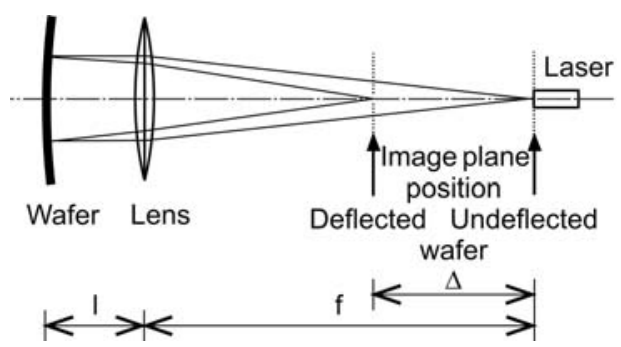

FIGURE 34. Optical scheme of wafers curvature measurement based on autocollimation principle. 
often the case, the reflected light will form an astigmatic beam. As it is known [56], two planes which contain the shortest and the longest radius of curvature, are perpendicular to each other. The corresponding curvatures are usually called tangential field of curvature $R_{T}$ and sagittal field curvature $R_{S}$. The quantity

$$
\frac{1}{R}=\frac{1}{2}\left(\frac{1}{R_{T}}+\frac{1}{R_{S}}\right)
$$

is their arithmetic mean value. Both the radii $R_{T}$ and $R_{S}$ can be determined experimentally, by searching for tangential as well as sagittal focal line spots. The information whether the measured curvature is concave or convex is defined by direction of focal spot shifting (toward or from the objective lens).

When speaking about the measurement sensitivity, such an autocollimation scheme is at the same level as interferometrical surface contour fringe pattern, moreover, also in this method in principle, photoelectric or CCD based focal spot position detection can easy be installed. The accuracy of the measurement is sufficient for reliable determination of residual stresses even on the samples $10 \div 20 \mathrm{~mm}$ in diameter. The stresses can be determined with reasonable precission of several MPa in a large range of measurement up to $10 \mathrm{GPa}$. One advancement more is the testing of materials, where their anisotropic properties have to be accounted for. Simple separation and determination of orthogonal curvatures as well as using of Eq. 7 provides good orientation in evaluation of orthotropic stress components [74]. A number of thermal/intrinsic residual stress measurements has been reported elsewhere [75-77].

Regarding the complexity of the task, determination of stress state in built-in components those that the MEMS structures are composed, has to be taken more seriously. Small dimensions of mechanical elements call for good sensitivity of measurement techniques and also for reliable quantifying of the values of displacements/deformations as well as loading forces in microscopic scale scenes. During the process of development of GaAs based microwave monolithic integrated circuits with integrated microthermal converter, three basic types of mechanical elements has been tested (Fig. 35). All the elements have the same or similar GaAs or GaAs/AlGaAs multilayers membrane structure (see Fig. 22a).

At first, the cantilever beam has been examined for steady state deformation. The overall cantilever structure is composed of three basic layers. GaAs and metal films Ti/Au. Unlike thin films on wafers, the layers have comparable thicknesses, that is why the Stoney's formula based interpretation of strain/stress state is not accessible and a more general solution for double-layer system must be employed. Such a solution is based on the Timoshenko theory of bi-metal thermostats [78]. Basic formulas for bi-layer strip component deformation

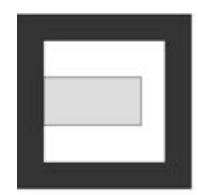

Cantilever

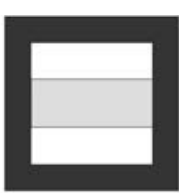

Bridge

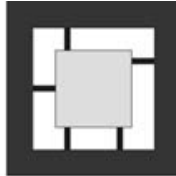

Island

FIGURE 35. Basic micromechanical elements tested on mechanical and/or thermo-mechanical characterization. 


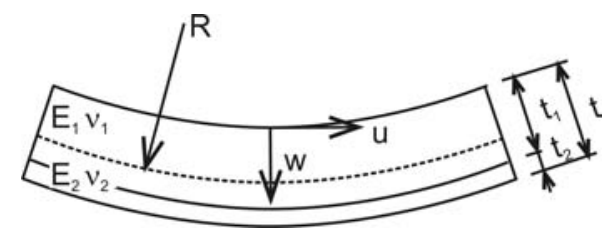

FIGURE 36. A schematic diagram of double-layer structure curving.

are presented in [79]

$$
\begin{gathered}
\frac{d^{2} w}{d x^{2}}=\frac{1}{R}=\frac{\varepsilon_{f}\left(t_{1}+t_{2}\right)}{2 \Theta\left(D_{1}+D_{2}\right)} \\
\Theta=\frac{1}{12}\left(\frac{t_{1}^{2}}{D_{1}}+\frac{t_{2}^{2}}{D_{2}}+\frac{3\left(t_{1}+t_{2}\right)^{2}}{D_{1}+D_{2}}\right) \\
D_{1}=\frac{E_{1} t_{1}^{3}}{12\left(1-v_{1}^{2}\right)} \\
D_{2}=\frac{E_{2} t_{2}^{3}}{12\left(1-v_{2}^{2}\right)}
\end{gathered}
$$

where $w, t_{1}, t_{2}$ are the quantities of double-layer geometry-deflection, thicknesses of the first and the second layer, respectively. $E_{1}, E_{2}$ and $v_{1}, v_{2}$ are the Young's modulus and Poisson's ratio, respectively, $D_{1}, D_{2}$ are the flexural rigidities (see Fig. 36). The value $\varepsilon_{f}$ is the free-standing strain, that is for thermal problem

$$
\varepsilon_{f}=\Delta \alpha_{t h} \Delta T
$$

where $\Delta \alpha_{t h}$ is the difference between coefficients of thermal expansion of the layers materials and $\Delta T$ is the temperature difference. The free-standing deformation induced by each of layer of the multilayer system is additive, then [78]

$$
\frac{1}{R}=\sum_{i=1}^{N} \frac{1}{R_{i}}
$$

For the specific case if the thickness $t_{1}$ of the film is much more thin than the thickness of the substrate $t_{2}$, the formulas (8)-(11) lead to the expression

$$
\frac{1}{R}=\frac{6 \varepsilon_{f} t_{1}}{t_{2}^{2}}
$$

that is for film stress $\sigma_{1}$ finaly the Stoney's formula is obtained

$$
\sigma_{1}=\frac{E_{2} t_{2}^{2}}{6 R t_{1}}
$$

Fig. 37 and corresponding plotted curves in Fig. 30 show the interference contours of the free cantilever deformation and varying cantilever profiles for different power heating. The thermal coefficient of cantilever curvature was obtained experimentally, analytically as well as numerically. The interpretation of experimental data was based on expression (8) using the GaAs thermal dilatation parameters [80]. Analytical simulation was based on the equation (12) and subsequently (8), and finaly the numerical simulation has been performed 


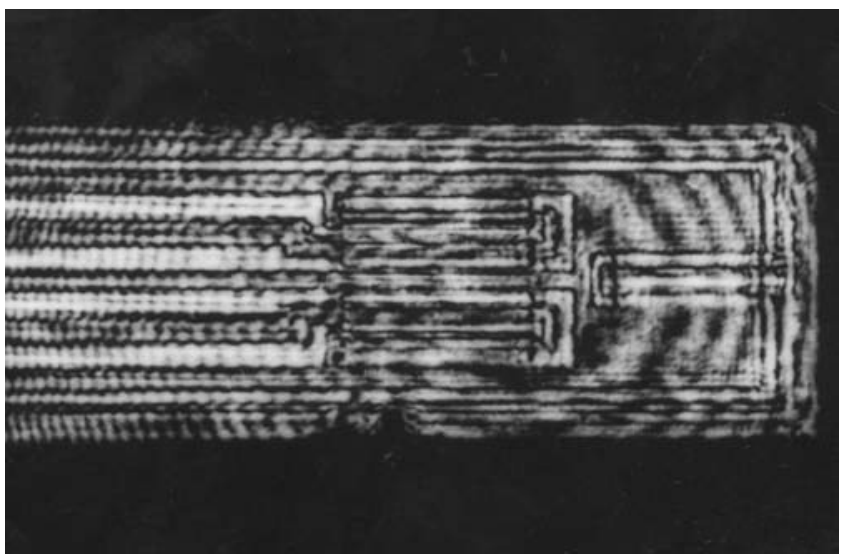

FIGURE 37. Interference pattern on GaAs/Ti/Au cantilever structure deformed after deposition process.

by solving steady-state, 2-D heat flow equation [50]. The coefficients values obtained are as follows

$$
\begin{array}{ll}
\mathrm{R}^{-1}=1.51 \mathrm{~m}^{-1} \mathrm{~K}^{-1} & \text { experimentally } \\
\mathrm{R}^{-1}=1.55 \mathrm{~m}^{-1} \mathrm{~K}^{-1} & \text { analytically } \\
\mathrm{R}^{-1}=1.60 \mathrm{~m}^{-1} \mathrm{~K}^{-1} & \text { numerically }
\end{array}
$$

As it is seen the coincidence of results is surprisingly good.

The deflection changes of free standing cantilever induced by thermal feeding of MTC has also been studied by detection of displacements at the tip of cantilever [14]. A narrow laser beam was focused onto the small area specularly reflected the beam back onto the detector (Fig. 38). The linear position sensitive detector (PSD) captures differences in electrical signals, thus creating the output $U$ related to the position of laser spot on the effective area. Since the moving of the light spot is in linear relation as well, then

$$
U \propto 2 \Delta \varphi
$$

where $\Delta \varphi$ is the change of surface inclination. At the small slopes of the surface deflection

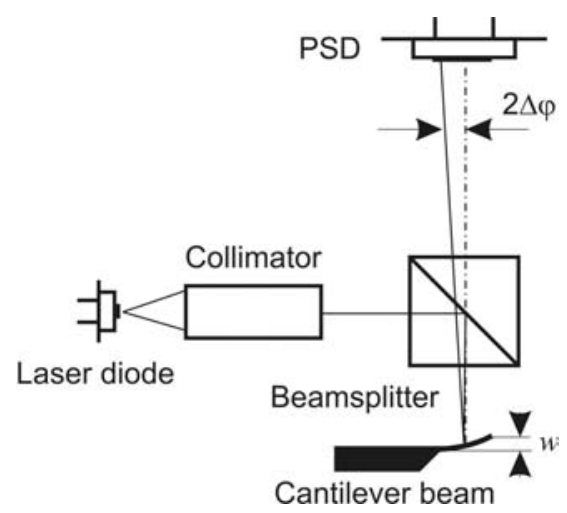

FIGURE 38. Schematic drawing of micro-cantilever deflection measurement by PSD. 
$w$ is related to lateral coordinate $x$

$$
U=K \cdot 2 \frac{\mathrm{d} w}{\mathrm{~d} x}
$$

where $K$ is the factor of proportionality-sensitivity value. At the experiment a good proportionality has been obtained in the relation deflection vs. heating power, in spite of considerable distortion of the reflected light spot upon the effective PSD area. It implies, that the accuracy of such a measurement is not affected strongly by this current effect. Another knowledge obtained was an excelent repeatability of the cantilever deformation changes. Such finding speaks in support of fact that the nature of residual stresses/ steady state deformation is thermal dilatation. Potential intrinsic stresses are predominantly induced by microstructural changes of interfaced materials and their mutual diffusion, that is why as a rule characteristic feature is their irreversibility during the thermal treating [81].

The Laser Doppler Vibrometer (LDV) is basically intended as a tool to measure periodic and, perhaps, stochastic vibrations, though the measurement principle of this technique permits its using for detection of one-shot events, too. Such an application has been carried out in order to observe the dynamic behaviour of GaAs technology based membrane microisland mechanical component (Fig. 35). At the experiments results of time dependent deflection development has been studied as a reaction to input step-wise electric/heating power. The effect of mechanical deformation follows from the mismatch of coefficients of thermal expansion. By the repeated loading the microisland area was mapped point by point to create a mesh of displacements perpendicular to the surface. Fig. 39a,b shows the rising and falling parts of transient microisland deformation thermal response in one of the points. In order to analyze the results measured we have taken into account as a first approximation the linearly proportional relationship between the out-of-plane deformation and actual temperature at the point of inspection by LDV. The relation was demonstrated numerically and it can be seen also from the comparison of measured temperature vs. time dependence on the thermal sensor as well. The latter assumption has led to the possibility of thermal-time response evaluation of MTC by means of deformation rates detecting.

Experimental determination of internal residual stresses by well defined loading is important particularly when the components or integrated parts of MEMS are fabricated as

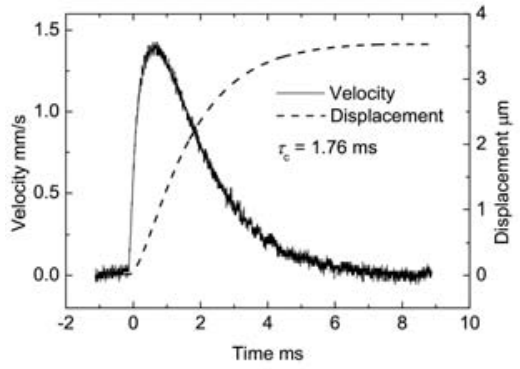

(a)

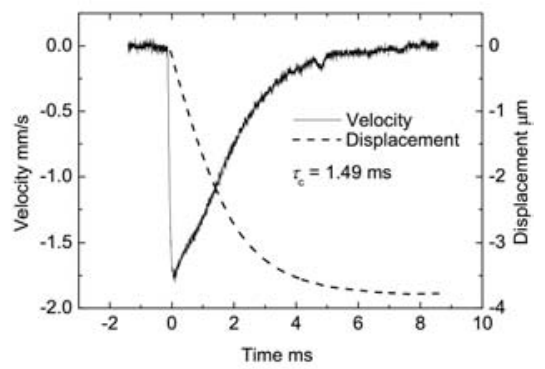

(b)

FIGURE 39. Rising (a) and falling (b) branches of transient thermal response of membrane micro-island on square-wave-shaped heat loading. 
membrane-like structures. Small dimensions of the mechanical components limit possibilities of tiny loading. Common, frequently used electrostatic mechanical exciting of static deformation as well as dynamic movings has inherent limitation consisting in a necessity of electric conductance of the surface to be loaded. Another approach is based on the employment of different thermal dilatation of MEMS materials by their heating or cooling. The observation of similarly actuated double (or three) layer element has been mentioned above. Besides the convenient heating the component under consideration can be heated by laser light energy as well [82]. In order to perform the laser beam excitation, mostly pulsed lasers are used. Typically laser pulses of Nd-YAG source $532 \mathrm{~nm}$ or $1064 \mathrm{~nm}$ are applied with an energy of $\mathrm{mJ}$ or even less which are sufficient to excite the measurable mechanical response. The energy of each pulse of short duration induces considerable increase of local surface temperature which is immediately followed by localy induced thermal dilatation. The interest for this loading method in micromechanics has to be increasing because of the possibility to excite very small objects. Nevertheless, against expectation, as a main drawbacks of the laser excitation may be regarded both badly controllable absolute value of loading as well as rather invasive way of interaction with the specimen tested involved in an eventual change of thermally dependent materials parameters.

Taking into account these opportunities and limitations, the loading through the acoustical coupling has been chosen to study the GaAs/AlGaAs membrane-like multilayer structure of microbridge (see above). The excitation of mechanical movement by sound can be regarded as desirable because of its tenderness and simple handling with both intensity and frequency adjusting. In vibrational analysis the acoustic excitation is currently used, however, the usual problem has arised, if the quantifying of actuating acoustic pressure values has to be done. To overcome the problem a procedure has been developed of the precise determination of acoustic pressure emitting by loudspeaker membrane by membrane vibration velocities measurement. This, in fact absolute calibration, is based on relationship between the velocity of longitudinally vibrating particles emitting by loudspeaker membrane and the periodic harmonic pressure changes $p_{s}$

$$
p_{s}=\rho c_{s} v
$$

where $v$ is the velocity of vibrations, $\rho$ is the air density and $c_{s}$ is sound velocity. In the nearest neighborhood of the vibrating loudspeaker membrane equal acoustic pressure can be admitted. For plane wave of sound the acoustic pressure and acoustic velocity are in phase that is Eq. (18) can be successfully applied to calculate the pressure. The use of Laser Doppler Vibrometer (LDV) is an ideal manner how to precisely gauge the velocities of loudspeaker membrane vibration, hence, the value of acoustic pressure can be accurately determined.

Another way how to tackle the task of controllable sound exciting is a generation of periodic harmonic changes of uniform pressure under the membrane bridge in a small chamber of pistonphone. Pistonphone is a device designed for microphone calibration and provides nominal sound level pressure of $118 \mathrm{~dB}$ at $173 \mathrm{~Hz}$. This value is related to 15.8 $\mathrm{Pa}$ (RMS) of the acoustic pressure which is in the range of several Pa to tens of Pa mostly adequate to experiments with micromechanical components.

Although the tension in a bridge membrane allows to be evaluated on the basis of measured resonant frequencies, the first resonant mode occurs at region of $60 \div 100 \mathrm{kHz}$ where the sound excitation is not very effective. On the other hand the expressive bulging oscillations of the microbridge are present at low sound frequencies where the loudspeaker 


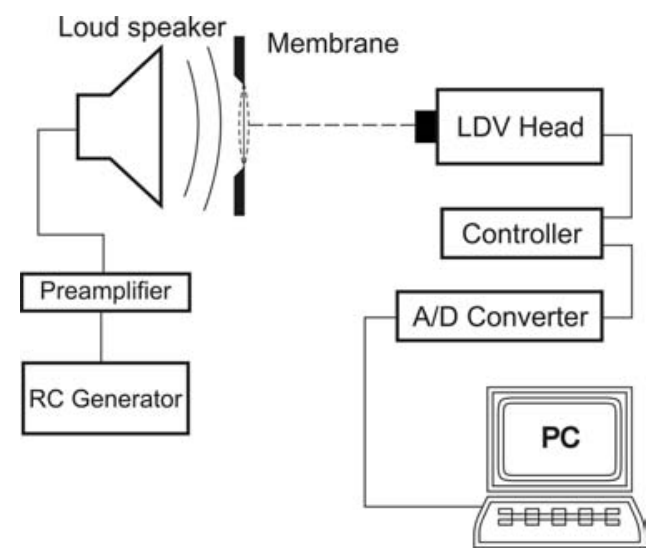

FIGURE 40. Experimental setup of membrane-like structures measurement by acoustic loading and Laser Doppler Vibrometer detecting.

actuation is very strong. The amplitudes of the oscillations are so high that the movements at hundreds of $\mathrm{Hz}$ show quasi-static nature with no inertial effect. Therefore, the formulas for static bulging of membrane-like bridge can be used to calculate the tension membrane stress knowing the value of acting acoustic pressure.

The schematic drawing of the Laser Doppler Vibrometry setup used to perform the bridge central deflection measurement is shown in Fig. 40. The maximum deflections measured varied in the range of $30 \mathrm{~nm}$ to $100 \mathrm{~nm}$, corresponding to values about $100 \div 120 \mathrm{~dB}$ of the acoustic pressure. Accordingly, the evaluated membrane tension was found to be $21 \mathrm{MPa}$. Other applications of optical techniques to characterise the mechanical behaviour of MEMS components can be found, for example, in [83-86].

Taking into account last years experience of many authors it can be stated that the optical methods had proven as a useful tool to solve many tasks and problems connected with mechanical and thermal characterisation of microcomponents and the structures of MEMS/M(O)EMS. The category of solvable tasks is very large and the development of new techniques and improvements is shown to be very promised for such purpose. The use of photoelectric signal reading permits the measurements to be high sensitive making the detection of deformation in microscale world feasible.

\section{MEMS Device Thermo-Mechanical Modeling}

The use of compound semiconductors (such as GaAs) for fabrication of MEMS addresses several problems. These materials are monocrystalline, have atomically flat interfaces, due to the technology of epitaxially grown layers, and extremely well controlled thickness, unlike polycrystalline materials. Also by controlling the lattice mismatch, the mechanical stress of epitaxial films is much more accurately controllable than in polycrystalline materials, which is usually controlled by annealing cycles. Performance and reliability are strongly affected by temperature causing thermal stress in multilayer structures. As temperature increases, physical changes within the device are accelerated. This 
seldom causes immediate, catastrophic failure, instead it brings about slow deterioration in the internal elements of the device, such as metallization areas, transistor junctions, temperature sensors, etc. The effect is cumulative, so failure rates could depend on the entire thermal history of the device. Temperature changes must therefore be analyzed carefully when designing a MEMS working on thermo-mechanical principle, not only for sensitivity optimization but also for reliability purposes.

The purpose of this part is to introduce the procedure for performing a thermomechanical analysis of thermal GaAs-based MEMS devices. It will provide the general procedure how thermal analysis should be made and model equations used to describe conduction, convection, radiation and mechanical effects caused by nonhomogenous temperature distribution. It also gives the values for thermal conductivity, heat transfer coefficients, emissivity, and reviews factors for various materials combined with GaAs technology. Increasing reliability requires both to control the temperature distribution in single elements of the device and to choose elements with high thermo-mechanical stress ratings adequate for the given application. The general doubt on the mechanical properties of compound semiconductors is largely speculative. While not as strong as silicon, compound semiconductors are sufficiently robust for most MEMS applications and are in fact stronger than the highest quality steel.

\section{GENERAL SIMULATION PROCEDURE}

The classical approach to the design and modeling of MEMS devices consists of three phases (Fig. 41):

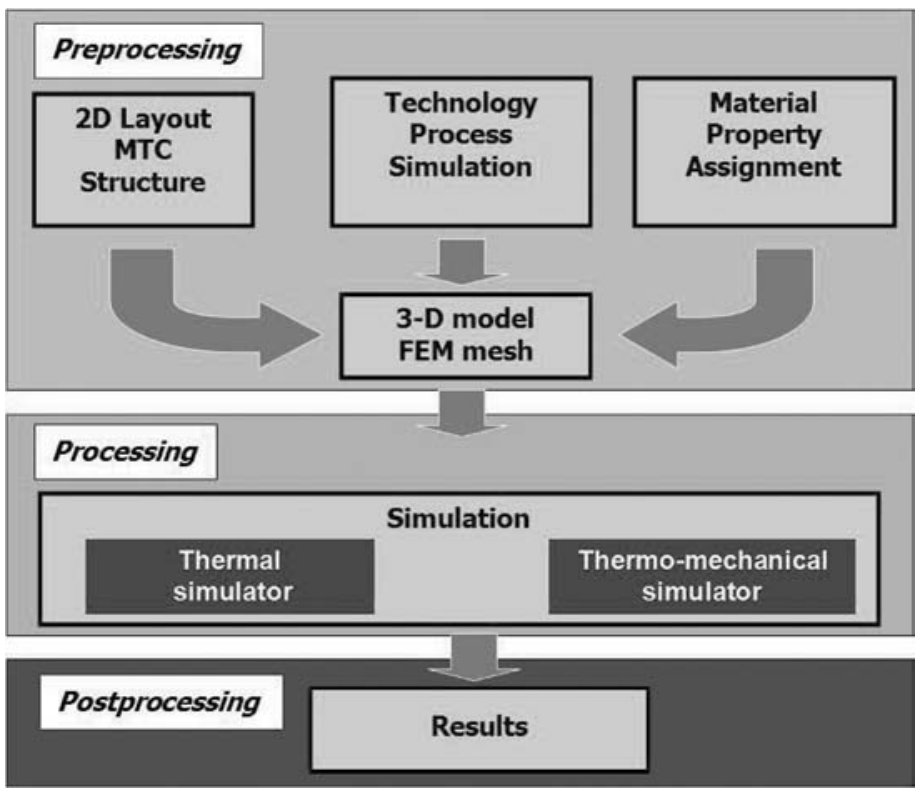

FIGURE 41. MEMS simulation general procedure. 
Preprocessing - Design of a proper model that is obtained in general from a 2D layout describing the shapes of particular working layers, and classification of the technological process. The technology process describes single deposition and etch step attributes, such as layer thickness, etching angle, etc. Material constants are assigned from a material database to each layer. The model is meshed for FEM simulators.

Processing-Boundary conditions assignment for particular walls of the model. Parameter setting for FEM simulator which should be combined in the so-called co-solve analysis.

Postprocessing_-Visualization and analysis of simulation results.

\section{CONSIDERATION OF COMPUTER-BASED SIMULATIONS}

- Computer simulation is used to solve a highly complex physical behavior of MEMS structures insolvable by analytical methods, or if the analytical solution were too simplifying.

- We can study the physical behavior of MEMS structures under steady state or time dependent boundary conditions. After designing a proper model and computing, in couple of hours (days) one can get the complex behavior of the whole structure, hence the procedure is much less time-consuming than technological realization.

- The experience one gets while designing the 3D model can contribute to a better design of the structure.

- The complex view of the studied problem on changing one design parameter allows observing the impact upon the whole structure.

- Once designed 3D models can be used in further designs or redesigns.

\section{GOVERNING EQUATIONS}

Steady-state and transient simulation of devices with embedded thermo-mechanical behavior entails sequential solving of three sets of differential equations governing the electric current (dissipation), thermal behavior, and thermo-elastic behavior.

First, the current distribution in the structure for specified voltage boundary conditions is determined by solving the following equation for continuity of current: Ohm's law in continuum form is written:

$$
J=\sigma \mathrm{E}
$$

where $J$ is the current density [A. $\mathrm{m}^{-2}$ ], $\mathrm{E}$ is the electric field, and $\sigma$ is the electric conductivity. For a complex solution it is necessary to solve the equation for current flow:

$$
\nabla \vec{J}+i_{v}=0
$$

Here $i_{v}$ is the current source per unit volume. The electric field can be expressed as $\mathrm{E}=$ $-\nabla V$, where $V$ is the electric potential (voltage).

Joule heat generation per unit volume is

$$
q=J \mathrm{E}
$$




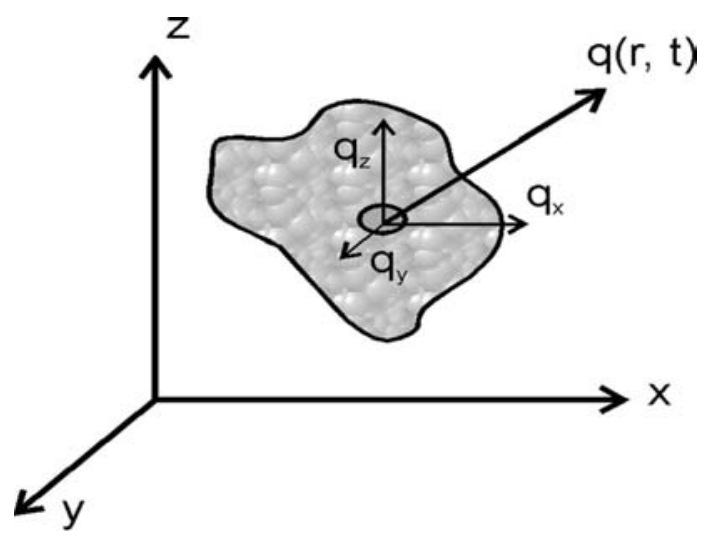

FIGURE 42. Heat flux definition on solid body.

The steady-state heat conduction equation shown below is solved for specified thermal boundary conditions imposed upon temperature and heat flux (including insulation, natural convection, and radiation). The Fourier equation for the distribution of temperature can be written as follows:

$$
\operatorname{div}(\lambda \operatorname{grad} T)=\rho c \frac{\partial T}{\partial t}-p
$$

where $\lambda\left[\mathrm{W} \mathrm{m}^{-1} \mathrm{~K}^{-1}\right]$ is the coefficient of thermal conductivity, $\rho\left[\mathrm{kg} \mathrm{m}^{-3}\right]$ is the density, $c\left[\mathrm{~J} \mathrm{~kg}^{-1} \mathrm{~K}^{-1}\right]$ is the thermal capacity, and $p\left[\mathrm{~W} \mathrm{~m}{ }^{-3}\right]$ is the specific heat. In the case of large temperature differences the coefficient of thermal conductivity is not constant, anyhow, in most MEMS applications it can be taken as constant.

The value of the heat flux can be expressed as:

$$
q=-\lambda \operatorname{grad} T \quad\left[\mathrm{~W} \cdot \mathrm{m}^{-2}\right],
$$

Fig. 42 shows a solid body placed in coordinates. The heat flux can be expressed as:

$$
q(r, t)=-\lambda \nabla T(r, t)
$$

Transcribing the above equation into Cartesian coordinates we get:

$$
\begin{aligned}
q_{x} & =-\lambda_{x} \frac{\partial T(x, y, z, t)}{\partial x} \\
q_{y} & =-\lambda_{y} \frac{\partial T(x, y, z, t)}{\partial y} \\
q_{z} & =-\lambda_{z} \frac{\partial T(x, y, z, t)}{\partial z}
\end{aligned}
$$

For isotropic materials $\lambda_{x}=\lambda_{y}=\lambda_{z}$. If the solid body is heated up by a constant power and cooled down constantly by the surrounding environment, then the temperature distribution will settle. For Cartesian coordinates the temperature distribution can be obtained by solving the following equation:

$$
\nabla^{2} T(r, t)+\frac{Q(r, t)}{\lambda}=\frac{1}{\alpha} \frac{\partial T(r, t)}{\partial t}
$$


$\alpha\left[\mathrm{m}^{2} / \mathrm{s}\right]$ in equation (28) is the thermal diffusivity and can be expressed as:

$$
\alpha=\frac{\lambda}{\rho c},
$$

The ambient of thermal MEMS devices are often various gases or liquids. Thus the convection effects should be also taken into account in some cases (it depends on specific dimensions and shapes of the device; in many cases convection is negligible). Heat transfer in gases or liquids has a physical nature different from that in a solid body. Individual particles can move mutually. The density of heat flux due to convection is given by [91]

$$
q=\alpha^{\prime} \Delta t=\alpha\left(t_{s t}-t_{t}\right) \quad\left[\mathrm{Wm}^{-2}\right],
$$

where $\alpha^{\prime}\left[\mathrm{W} \mathrm{m}{ }^{-2} \mathrm{~s}^{-1}\right]$ is the heat transfer coefficient given by the criteria equation (see below), $t_{s t}$ is the wall temperature of the solid body, $t_{t}$ is the temperature of the surrounding gas or liquid, and $A$ is the contact area. The criteria equation can be found in literature [105] for instance in the following form:

$$
N u=f(\operatorname{Re}, G r, \operatorname{Pr}, \ldots), \quad \text { where }
$$

$\mathrm{Nu}, \mathrm{Re}, \mathrm{Gr}$ and $\mathrm{Pr}$ are the Nusselt, Reynolds, Grashof and Prandtl numbers, respectively. The criteria equation for natural convection can be expressed in the form:

$$
N u=C \cdot(G r \cdot \operatorname{Pr})^{n},
$$

where $C$ and $n$ depend on the value of the product Gr.Pr according to Tab. 2. For MEMS devices operating at room temperature the heat loses caused by radiation can be usually neglected. On the other hand, radiation can have a significant effect for devices working much above $400 \mathrm{~K}$. Therefore for such devices verification of the radiation effect should be performed. Heat losses caused by radiation are given by the Stefan-Boltzmann emission law:

$$
P_{\text {Rad }}=\varepsilon_{1,2} \cdot C_{0} \cdot A \sigma_{S B} T^{4} \quad[\mathrm{~W}]
$$

where

$$
\varepsilon_{1,2}=\frac{1}{\frac{1}{\varepsilon_{1}}+\frac{1}{\varepsilon_{2}}-1}
$$

$\varepsilon$ is the emissivity of gray body, $A$ is the area of the body, and $\sigma_{S B}$ is the Stefan-Boltzmann constant $5.67 \times 10^{-8} \mathrm{Wm}^{-2} \mathrm{~K}^{-4}$.

The third and final step in the simulation is to solve the elastic equilibrium equations under temperature induced thermal strain. In the linear theory of elasticity, when the deformation are small the dependence between the strain and stress tensors is given by the

TABLE 2. Value of $\mathrm{C}$ and $\mathrm{n}$ depends on Gr.Pr

\begin{tabular}{lll}
\hline Gr.Pr & C & \multicolumn{1}{c}{$\mathrm{n}$} \\
\hline$<1.10^{-3}$ & 0,45 & 0,0 \\
$1.10^{-3} \cong 5.10^{2}$ & 1,18 & 0,125 \\
$5.10^{2} \cong 2.10^{7}$ & 0,54 & 0,25 \\
$2.10^{7} \cong 1.10^{13}$ & 0,195 & 0,333 \\
\hline
\end{tabular}




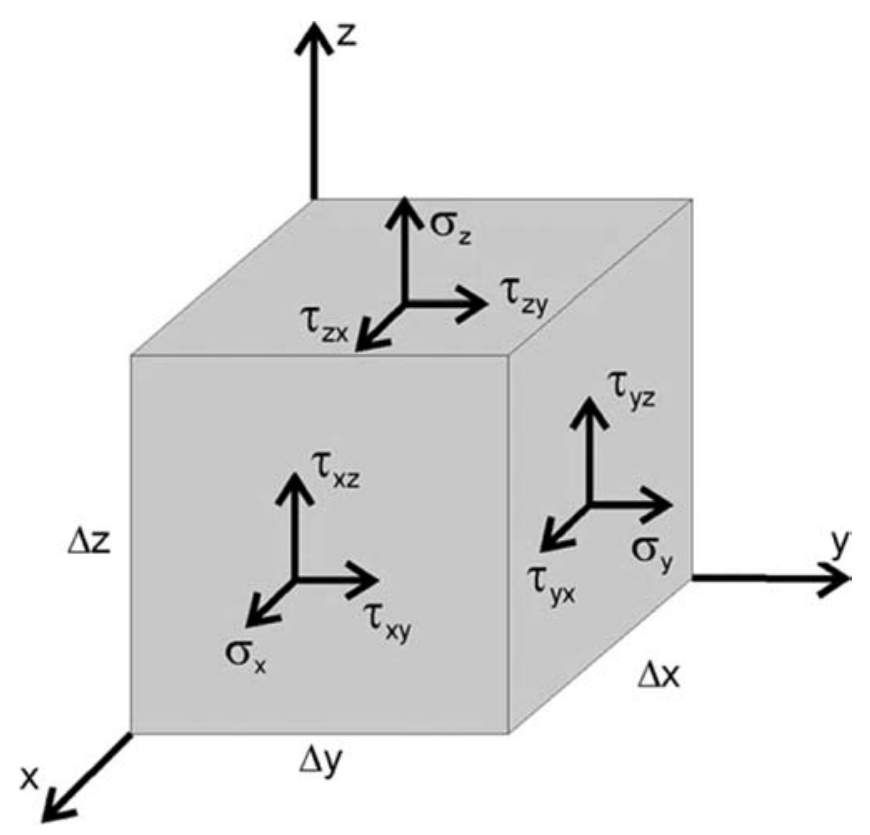

FIGURE 43. Mechanical stresses and shear stresses affecting the solid body.

Hook's law [90]

$$
\begin{aligned}
\delta_{x} & =\frac{1}{E}\left[\sigma_{x}-v\left(\sigma_{y}+\sigma_{z}\right)\right] \\
\delta_{y} & =\frac{1}{E}\left[\sigma_{y}-v\left(\sigma_{z}+\sigma_{x}\right)\right] \\
\delta_{z} & =\frac{1}{E}\left[\sigma_{z}-v\left(\sigma_{x}+\sigma_{y}\right)\right]
\end{aligned}
$$

Here $E$ is the Young's modulus, $\sigma_{x}, \sigma_{y}$ and $\sigma_{z}$ are the mechanical stresses in mutually orthogonal directions acting perpendicularly to the wall of the body (Fig. 43), $v$ is the Poisson's ratio. Mechanical structures in the case of the linear theory of elasticity have idealized some physical properties by the following assumptions: homogeneous body, isotropic, linearly elastic in all points.

By action of external forces and non-homogeneous temperature distribution, the elastic body is deformed and internal forces are induced. In a linear elastic continuum, displacements appear defined by Lamé equation:

$$
(\varphi+\psi) \operatorname{grad}(\operatorname{div} \delta)+\psi \Delta \delta-(3 \varphi+2 \psi) \alpha_{T} \operatorname{grad} T+f-0
$$

where both $\varphi \geq 0$ and $\psi>0$ are the so-called Lamé coefficients given by material parameters

$$
\varphi=\frac{v \cdot E}{(1+v) \cdot(1-2 v)}, \quad \psi=\frac{E}{2(1+v)},
$$

where $E(r, T)[\mathrm{MPa}]$ is the Young's modulus, $v(r, T)$ is the Poisson's coefficient, $\delta(r)[\mathrm{m}]$ 
and $f(r, \ldots)\left[\mathrm{Nm}^{-3}\right]$ are the vector of displacement and the vector of internal volume forces, respectively.

\section{BOUNDARY CONDITIONS}

Varying thermal and mechanical boundary conditions can significantly affect the analysis results of thermo-mechanical MEMS devices. Mechanical boundary conditions define how the device is constrained from movement. Mechanical conditions can be considered fixed for a given MEMS device. Thermal boundary conditions, such as conduction, convection, and radiation, on the other hand, depend on the surroundings, packaging, etc. That is why careful assessment of thermal boundary conditions is necessary.

Precise thermal analysis that includes the conduction, convection and radiation effects is needed to properly predict the behavior of thermo-mechanical devices. Not only quantitative but also qualitative performance can be changed provided that the thermal boundary conditions are not modeled correctly. In many cases convection and radiation losses from the device could be negligible and heat dissipation is entirely due to the heat lost to the substrate. It depends on the shape and dimensions of the device. It can be modeled as a constant ambient temperature condition at the base of the substrate or on the sidewalls of the model. Such boundary conditions are known as Dirichlet. The aforementioned assumption may not be true when the thermal mass of the substrate is not large enough to preserve the ambient temperature. It could happen when an array of thermo-mechanical devices is used. At that moment a natural boundary condition (Neumann boundary condition) must be chosen. After completing the thermal analysis we can get no uniform temperature distribution at the substrate. The technology process, environment, and packaging of the MEMS device are the factors of appropriate boundary conditions decision. The choice of the type of boundary conditions could significantly affect the device behavior.

\section{3D MODEL}

The micromechanical structures used in thermally based MEMS devices are mostly designed as free standing structures. To increase the thermal resistance values, they have to be designed with the thickness as thin as possible. Moreover, optimization of the micromechanical structure dimensions, particularly of the aspect ratio between the structure length that increases the thermal resistance and structure thickness, has to be carried out to find the best trade-off between the thermal resistance and acceptable mechanical stress.

The main design criterions, such as high electro-thermal conversion efficiency, linearity, short response time, thermal stability, micromechanical integrity and integration device simplicity should be taken into account in the MTC design.

Using the FEM simulation tools, three different models of GaAs MTC have been designed. The first model represents two symmetrical cantilever beam structures $(350 \mu \mathrm{m}$ long and $120 \mu \mathrm{m}$ wide) fixed by a polyimide layer in a rigid GaAs substrate rim. The rim has been designed $10 \mu \mathrm{m}$ thick and $200 \mu \mathrm{m}$ wide for the purpose of thermal and thermomechanical simulations (Fig. 44). These dimensions ensure a sufficient mass for simulator boundary condition setting while keeping the number of simulation nodes at a reasonable 


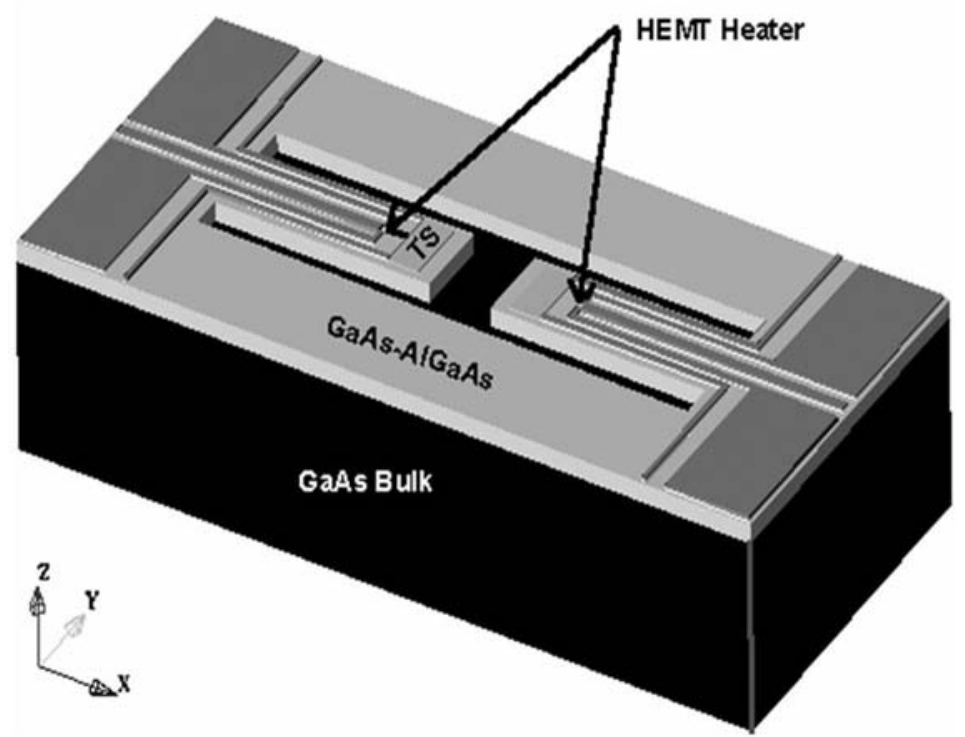

FIGURE 44. Fixed cantilever based MEMS device. Temperature sensors are placed on the end of cantilever where we get uniform temperature distribution. Ti/Au metallization lines are implicated in this model. Polyimide is not visible. Length of cantilever is $350 \mu \mathrm{m}$.

level. The meander-shaped temperature sensor TS has been placed at the free end of the cantilever in order to achieve the highest thermal sensitivity. The position of the HEMT heater is next to the TS.

Fig. 45 demonstrates another promissing GaAs island structure that has been proposed to increase the sensor thermal resistance. The GaAs island floats in a $1 \mu \mathrm{m}$ thin polyimide

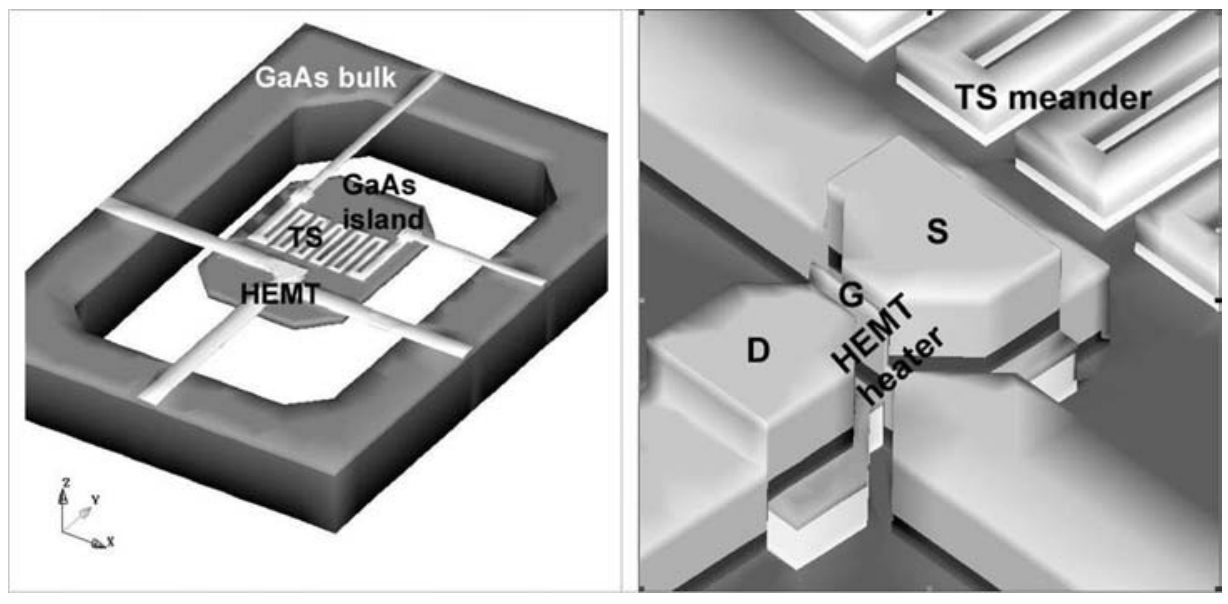

FIGURE 45. Model of island based MTC device. GaAs island is "floating" in a $1 \mu \mathrm{m}$ thick Polyimide layer (not visible). Next view is the detail of MESA etched HEMT heater. The meander-shaped TS is also shown. Z-direction is 20 times magnified. 
TABLE 3. Particular steps to create 3-D model of MTC island structure

\begin{tabular}{|c|c|c|c|c|c|}
\hline Step & $\begin{array}{l}\text { Technology } \\
\text { process }\end{array}$ & Material & $\begin{array}{l}\text { Layer thickness } \\
\qquad[\mu \mathrm{m}]\end{array}$ & Mask & $\begin{array}{c}\text { Deep of etching } \\
{[\mu \mathrm{m}]}\end{array}$ \\
\hline 1 & Base & GaAs & 10 & Substrate & \\
\hline 2 & Etch & GaAs & & MEMS & 10 \\
\hline 3 & Etch & GaAs & & MTC & 10 \\
\hline 4 & Deposit & BPSG & 0 & & \\
\hline 5 & Deposit & GaAs & 1 & & \\
\hline 6 & Etch & GaAs & & MEMS & 1 \\
\hline 7 & Deposit & PolySi & 0.03 & & \\
\hline 8 & Etch & PolySi & & TS & 0.03 \\
\hline 9 & Deposit & Platinum & 0.06 & & \\
\hline 10 & Etch & Platinum & & TS & 0.06 \\
\hline 11 & Deposit & InGaAs & 0.03 & & \\
\hline 12 & Etch & InGaAs & & MESA & 0.03 \\
\hline 13 & Etch & InGaAs & & Gate & 0.03 \\
\hline 14 & Deposit & Titanum & 0.05 & & \\
\hline 15 & Etch & Titanum & & Metalization & 0.05 \\
\hline 16 & Deposit & Gold & 0.15 & & \\
\hline 17 & Etch & Gold & & Metalization & 0.15 \\
\hline 18 & Deposit & Polyimide & 1 & & \\
\hline 19 & Etch & Polyimide & & Substrate & 1 \\
\hline 20 & Sacrifice & BPSG & & & \\
\hline
\end{tabular}

layer. The polyimide membrane $(225 \mu \mathrm{m} \times 360 \mu \mathrm{m})$ mechanically fixes and thermally isolates the GaAs island membrane which is $175 \mu \mathrm{m}$ long and $125 \mu \mathrm{m}$ wide. The GaAs substrate rim has been designed $10 \mu \mathrm{m}$ thick and $50 \mu \mathrm{m}$ wide analogous to the previous model. Tab. 3 summarizes particular steps with parameters used in a model of the island MTC structure. solid models were elaborated in detail, e.g., the HEMT heater and the temperature sensor shapes represent real micromachined structures (Fig. 45). Thermal constants and layer thickness used for MTC modeling are summarized in Tab. 2.

Meshing is the most important step in the simulation, since it affects the accuracy of the results. The Merge Layers and Extrude option have been used to merge and extrude all layers in order to produce a continuous brick mesh of non-orthogonal model shapes. Considering accurate solutions of movable parts, parabolic 27-node elements have been chosen [49]. To reduce the number of nodes, thereby the computing time, we created gradation towards small features in the models and larger elements in the open spaces. These settings are recommended for mechanical simulations where high stresses are located at small features. All designed models contained approximately 120,000 nodes. In principle, growth of density of nodes is followed by more accurate results. On the other hand, there is a trade-off between the accuracy and the simulation time.

\section{SIMULATIONS}

Thermo-mechanical numerical modeling and simulation has a significant influence on the optimum topology of the MTC design. The main characteristics for optimization of these 
devices are the temperature distribution over the sensing area, the time response, sensitivity and mechanical stresses induced in the multilayer structure.

Three MTC device types have been investigated to compare their thermal and mechanical behaviour, free standing cantilever, bridge as well as microisland (see above). The temperature distribution caused by power dissipation in the heater and the thermal time response as a result of power changes were evaluated by the MemTherm module, and the mechanical stresses, displacements and deformations were simulated using the thermomechanical modules MemMech. The input power dissipation in the heater for the simulation process was defined by the heat flux through the HEMT gate area $(10 \mu \mathrm{m} \times 0.5 \mu \mathrm{m})$. We can use this approximation because the heat dissipation in HEMT structure is positioned in a very thin InGaAs conduction layer formed under the gate area (see Fig. 12).

\section{STEADY STATE THERMAL ANALYSIS AND P-T CHARACTERISTIC}

In an isotropic homogeneous material the steady state heat equation can be written as:

$$
\nabla^{2} T \equiv \frac{\partial^{2} T}{\partial x^{2}}+\frac{\partial^{2} T}{\partial y^{2}}+\frac{\partial^{2} T}{\partial z^{2}}=-\frac{1}{k} Q(x, y, z)
$$

where $Q$ represents the generated internal heat, $k$ denotes the thermal conductivity, $c_{p}$ is the specific heat, and $T$ is the temperature.

In order to complete the specification of the thermal simulations, it is necessary to specify the boundary conditions. For the thermal analysis problem, the essential boundary conditions are prescribed by temperatures. Furthermore, the conductive heat flux and the radiation boundary conditions may also be applied. The spatial temperature distribution of the MTCs and steady state heat flux were calculated taking into account the heat transfers to infinity. In the current analysis, according to application requirements, the fixed thermal boundary is defined for all sidewalls of the GaAs substrate. These sides were kept at room temperature of $300 \mathrm{~K}$ while other sides were adiabatic. The CoventorWare simulation manager (SimMan) was used to investigate the influence of power dissipation in the heater.

Plots give a good overall visualization of the temperature distribution (Fig. 46) in the island MTC structure. Shading and z-direction value represent the temperature distribution for $1 \mathrm{~mW}$ power dissipation from the HEMT heater. The island is "floating" in the polyimide layer that mechanically and thermally isolates the MTC structure. The polyimide layer is not shown but was considered in the simulation. Analyses were performed for both vacuum ambient and non-convective gaseous medium around the MTC structure. Heat losses due to radiation were taken into account too, in the simulation but were found to be negligible. Thermal material properties choised are summarized in Tab. 4.

The power-to-temperature $(P-T)$ conversion characteristics of the MTC structures have been investigated and they were also compared with that of real micro-machined devices. Figure 47 shows the simulated $P-T$ conversion characteristics of an island-based MTC device in direct comparison with both the fixed cantilever and bridge based MTC components. The slope of the $P-T$ curves determines the thermal resistance values, $R_{\mathrm{th}}$. The benefit from the improved electro-thermal conversion efficiency of the island structure is clearly visible. Thermal resistance as high as $24 \mathrm{~K} / \mathrm{mW}$ has been achieved, which is 
TABLE 4. Selected thermal material properties

\begin{tabular}{lccccl}
\hline & $\begin{array}{c}\text { Thickness of } \\
\text { deposited } \\
\text { layer }[\mu \mathrm{m}]\end{array}$ & $\begin{array}{c}\text { Thermal expansion } \\
\text { coefficient }\left[\mathrm{m} . \mathrm{K}^{-1}\right]\end{array}$ & $\begin{array}{c}\text { Thermal conductivity } \\
{\left[\mathrm{W} . \mathrm{m}^{-1} \mathrm{~K}^{-1}\right]}\end{array}$ & $\begin{array}{c}\text { Specific heat } \\
{\left[\mathrm{J} . \mathrm{kg}^{-1} \cdot \mathrm{K}^{-1}\right]}\end{array}$ & Mark \\
\hline Material & 10 & $6.8^{*} 10^{-6}$ & 46 & 351 & Substrate \\
GaAs & 1 & $6.8^{*} 10^{-6}$ & 46 & 351 & MTC layer \\
PolySi & 0.03 & $4.7^{*} 10^{-6}$ & 148 & 107 & Temperature Sens. \\
Platinum & 0.06 & $8.9^{*} 10^{-6}$ & 71.6 & 133 & Temperature Sens. \\
GaAs & 0.02 & $6.8^{*} 10^{-6}$ & 46 & 351 & HEMT heater \\
Titanum & 0.05 & $1.0^{*} 10^{-5}$ & 21.9 & 528 & Metalization \\
Gold & 0.15 & $1.41^{*} 10^{-5}$ & 267 & 129 & Metalization \\
Polyimide & 1 & $6.0^{*} 10^{-6}$ & $1.46^{*} 10^{-1}$ & 510 & Mech. Fixing \\
\hline
\end{tabular}

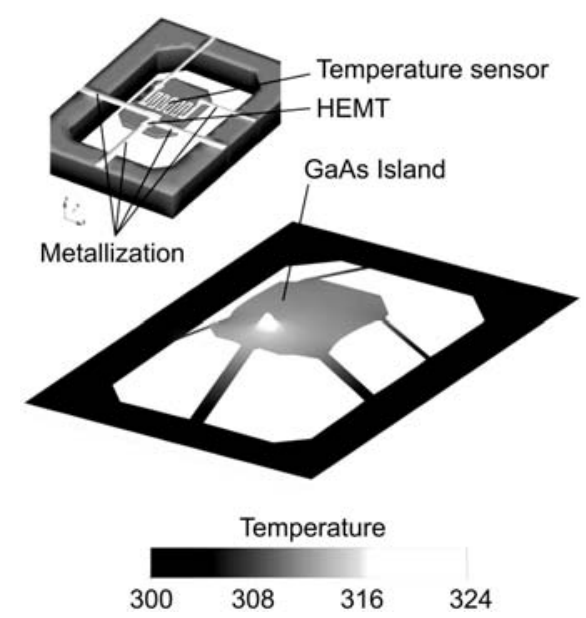

FIGURE 46. 3-D plots of temperature distribution of island based MTC device. The island is "floating" in polyimide layer that mechanically and thermally isolates the MTC structure. Polyimide not shown.

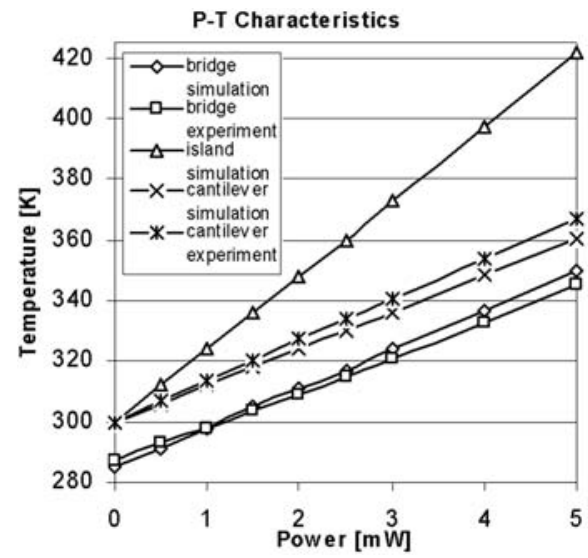

FIGURE 47. Simulated island, cantilever and bridge P-T conversion characteristics. Comparison with real micromachined MTC device. Ambient temperature for bridge MTC was $285 \mathrm{~K}$ whereas other two MTCs ambient temperatures were $300 \mathrm{~K}$. 
The power on/off transient characteristics

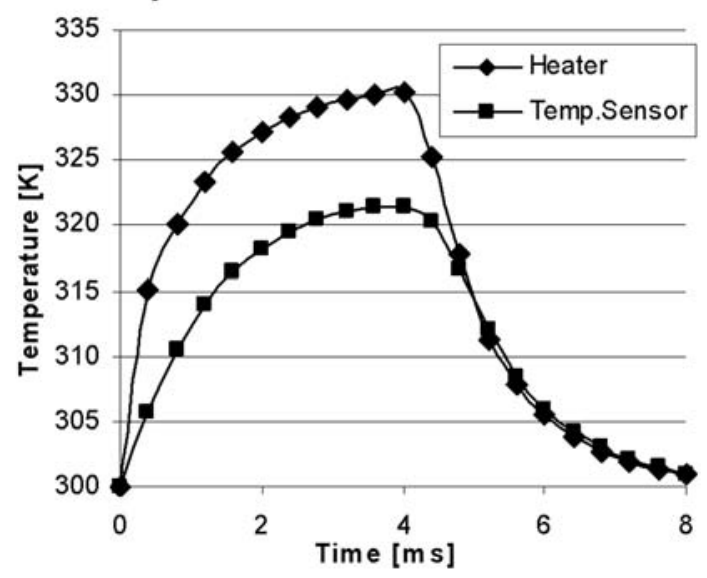

FIGURE 48. The simulated power on/off transient characteristics for island MTC structure for power ON of $1 \mathrm{~mW}$. At the beginning there was power of $1 \mathrm{~mW}$ switched ON. In the time of $4 \mathrm{~ms}$ the power was switched OFF.

two-times higher than that of the bridge-based MTC element $(11.5 \mathrm{~K} / \mathrm{mW})$. When compared with experiment, the thermal resistance values are congruent.

\section{TEMPERATURE TRANSIENT ANALYSIS}

The transient thermal response characteristics evaluation of the MTC structures mostly can be regarded as essential. Instantaneous temperature distribution on the body of MTC can be obtained solving the thermal transient equation:

$$
\frac{\partial T}{\partial t}=\frac{k}{\rho c_{p}} \nabla^{2} T
$$

where $\rho$ denotes the density of the material and $k$ its thermal conductivity. Thermal boundary conditions has been applied the same as for steady-state analysis. Additionally, the temperature of the MTC body was defined at time $t=0$ to be $300 \mathrm{~K}$.

Simulated transient on/off power characteristics for an island structure are depicted in Fig. 48. At the beginning a power of $1 \mathrm{~mW}$ was switched ON. After $4 \mathrm{~ms}$ the power was switched OFF. The thermal time constant obtained as $1.5 \mathrm{~ms}$. There are two transients in Fig. 48. The upper one is the temperature of the heater and the bottom dependence shows the average temperature of the TS. The thermal time constant of the cantilever beam arrangement is $1.7 \mathrm{~ms}$, which is nearly consistent with that of the island MTC structure.

\section{STRESS AND DISPLACEMENT EVALUATION}

As was noted above, mechanical stresses can have a great influence on the mechanical as well as electrical properties. The initial residual stresses caused by temperature differences during membrane layers deposition were evaluated analytically and experimentally [87]. 


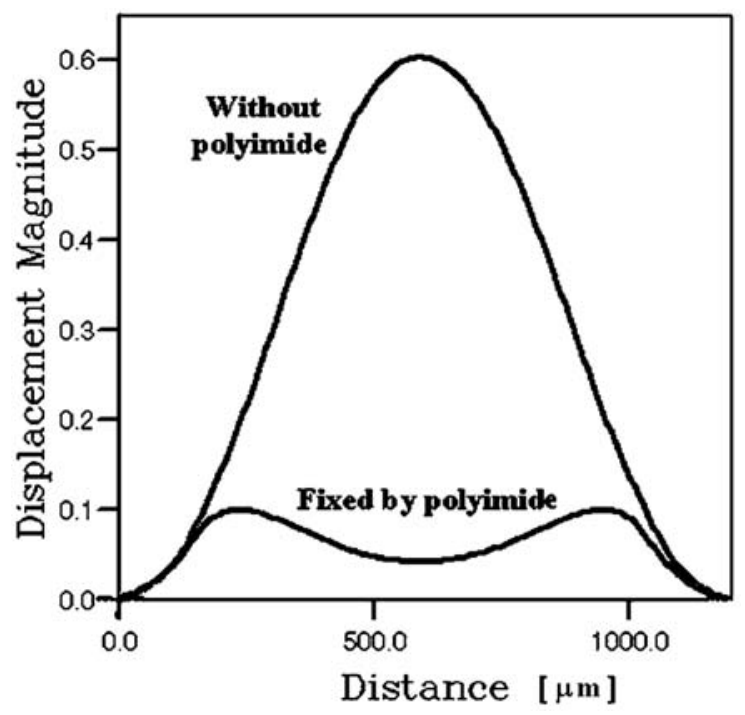

FIGURE 49. Displacement magnitude (in $\mu \mathrm{m}$ ) along the length of the bridge in $\mathrm{Z}$ direction caused by initial stress in metallization. Comparison between bridge fixed by polyimide layer and bridge where polyimide layer was removed is shown. A cross section was made in the middle of the bridge in $\mathrm{X}$-axes direction.

Analytical calculation has been performed using a simple analytical expression (12). The initial stress in the metallization (before bridge etching) for temperature difference $\Delta T=170 \mathrm{~K}$ was calculated 81.6 MPa for Ti and 51.3 MPa for Au layer, respectively. Thus, after the etching of GaAs substrate base, the mean stress throughout the thickness of membrane bridge structure is about $25 \mathrm{MPa}$. The experimental measurement by the detection of the membrane deflection amplitudes as a response to acoustic pressure gives the value of the mean membrane stress aproximately $21.6 \mathrm{MPa}$ (see above). As seen, the result is consistent well with the simple analytical calculation [19].

Figure 49 shows the displacement magnitude in z-axis direction along the length of the bridge caused by initial stress in $\mathrm{Au} / \mathrm{Ti}$ metallization. Comparison is shown between the bridge fixed by a polyimide layer and the bridge where the polyimide layer was removed. A cross section was drawn in the middle of the beam in $\mathrm{x}$-axis direction. As it can be seen the polyimid film reduces strongly the deformation of this mechanical component.

In order to model MTC devices, the combination of the heat conduction equations with the linear elasticity has to be done. Mechanical and thermal boundary conditions were defined for the sidewalls of the GaAs substrate. These sides were kept at room temperature $300 \mathrm{~K}$ while other sides were adiabatic and were set as rigid, i.e., immobile. The initial stress was set in each material according to the analytical calculation.

The stress and displacement magnitude were simulated using MemMech simulator. The mechanical material properties are summarized in Tab. 5. Figure 50 shows the plot of residual stresses and the deformation of the island structure caused by heating. Shading represents the residual stress for $1 \mathrm{~mW}$ power dissipation in the heater. The biggest stresses (520 MPa) are located in the place of the meander-shaped PolySi temperature 
TABLE 5. Selected mechanical material properties

\begin{tabular}{lccccl}
\hline Material & $\begin{array}{c}\text { Young modulus } \\
{[\mathrm{GPa}]}\end{array}$ & $\begin{array}{c}\text { Poisson } \\
\text { coefficient } \\
{[-]}\end{array}$ & $\begin{array}{c}\text { Density } \\
{\left[\mathrm{kg} \cdot \mathrm{m}^{-3}\right]}\end{array}$ & $\begin{array}{c}\text { Mechanical } \\
\text { stress } \\
{[\mathrm{MPa}]}\end{array}$ & \multicolumn{1}{c}{ Mark } \\
\hline SI-GaAs & 85 & 0.31 & 5370 & - & Substrate \\
GaAs & 85 & 0.31 & 5370 & - & MTC layer \\
PolySi & 160 & 0.22 & 2230 & - & Temperature Sensor \\
Platinum & 145 & 0.35 & 21400 & - & Temperature Sensor \\
GaAs & 85 & 0.31 & 5370 & - & HEMT heater \\
Titanum & 120 & 0.33 & 4506 & $81.6^{1}$ & Metalization \\
Gold & 78 & 0.29 & 19300 & $51.34^{2}$ & Metalization \\
Polyimide & 2.4 & 0.3 & 1100 & - & Mechanical fixation \\
\hline
\end{tabular}

sensor. The MTC structure is fixed by a polyimide layer that is not shown in the figure but was taken into account during simulation. The scale magnification in z-direction is $10 \times$ and the displacement magnification is $2 \times$. The maximum stress has been located in $\mathrm{Si} / \mathrm{Pt}$ meander-shaped TS. For power dissipation of $3 \mathrm{~mW}$, the TS temperature is $375 \mathrm{~K}$ and the mechanical stress reaches up to $900 \mathrm{MPa}$. These values of residual MTC stresses, however, have no significant influence upon the micromechanical integrity of the MEMS device.

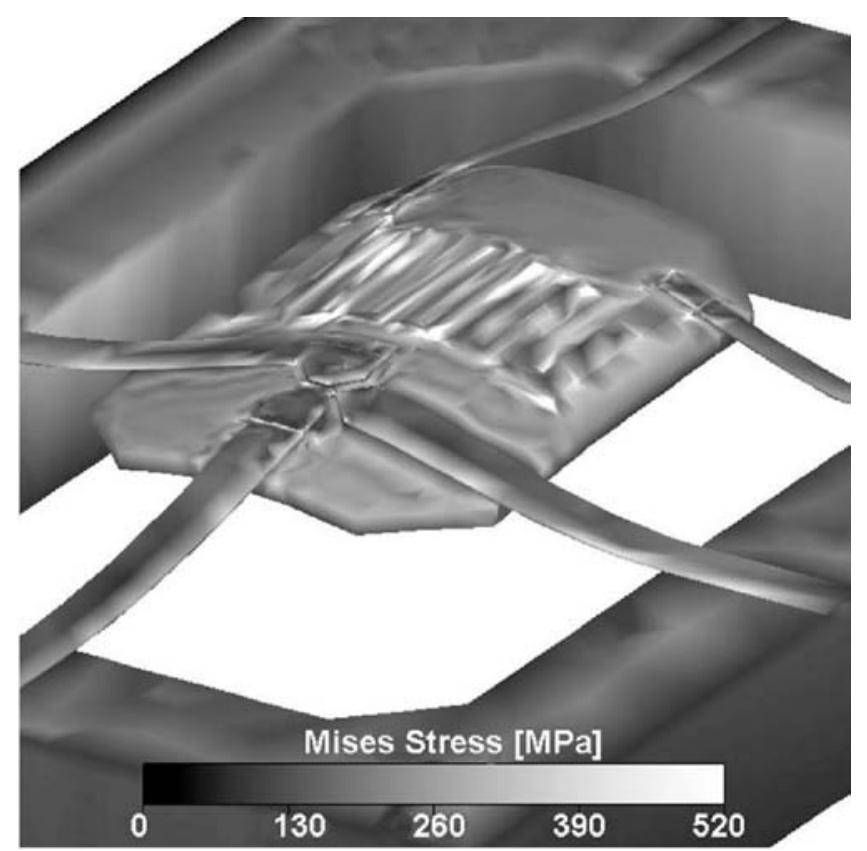

FIGURE 50. Residual stresses and deformation of the island structure caused by heating up with power dissipation of $1 \mathrm{~mW}$ in the heater. Polyimide layer was investigated in simulation (not visible). Values of stress are in MPa. 
TABLE 6. Comparison of the designed island based MTC devices (Island structure suspended with and without GaAs bridge)

\begin{tabular}{|c|c|c|c|}
\hline & $\begin{array}{c}\text { Island without } \\
\text { GaAs }\end{array}$ & $\begin{array}{c}\text { Island with } \\
\text { GaAs }\end{array}$ & $\begin{array}{l}\text { Optimized island } \\
\text { with GaAs }\end{array}$ \\
\hline $\mathrm{R}_{\mathrm{th}}$ simulation $[\mathrm{K} / \mathrm{mW}]$ & 24 & 13 & 26 \\
\hline $\mathrm{R}_{\mathrm{th}}$ measurement $[\mathrm{K} / \mathrm{mW}]$ & - & 14 & - \\
\hline$\tau$ simulation $[\mathrm{ms}]$ & 0.9 & 0.9 & 0.8 \\
\hline$\tau$ measurement $[\mathrm{ms}]$ & - & 0.8 & - \\
\hline Max. temperature $[\mathrm{K}](1 \mathrm{~mW})$ & 332 & 320 & 336 \\
\hline Max. displacement $[\mu \mathrm{m}][1 \mathrm{~mW}]$ & 2.74 & 0.26 & 5.28 \\
\hline Max. mechanical stress [Mpa] (1 mW) & 540 & 434 & 284 \\
\hline
\end{tabular}

\section{INFLUENCE OF THE GATE WIDTH ON MAXIMAL TEMPERATURE OF MTC STRUCTURE}

The influence of the gate width on the maximum temperature of MTC structure has been simulated. Temperature distributions in the HEMT and in the MTC structure for different gate widths $(5 \mu \mathrm{m}, 10 \mu \mathrm{m}, 15 \mu \mathrm{m}, 20 \mu \mathrm{m})$ have been obtained. From the simulation results it follows that the maximum temperature of the MTC microstructure which is located in the gate of the HEMT is inversely proportional to the gate width (see Fig. 51 and Fig. 52).

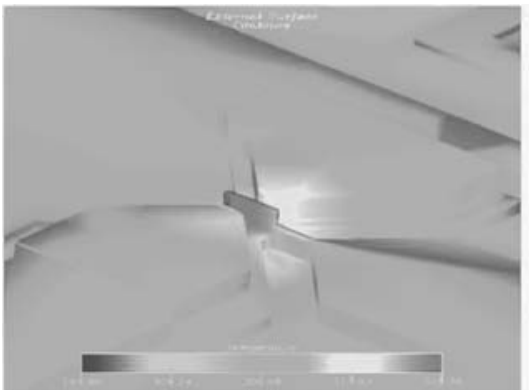

(a)

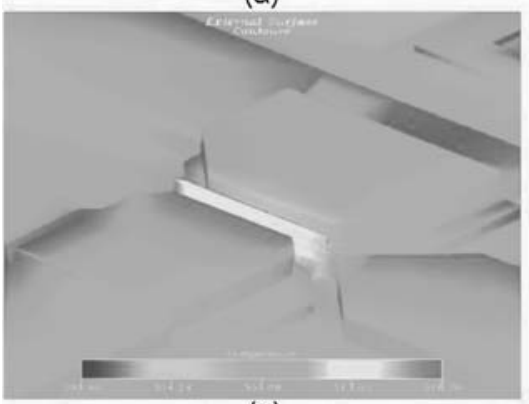

(c)

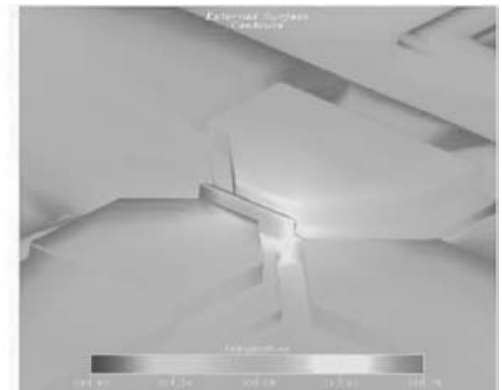

(b)

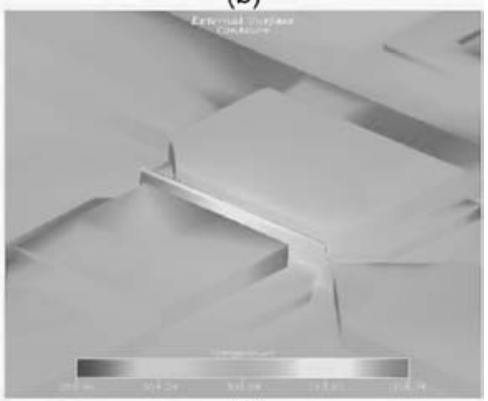

(d)

FIGURE 51. Temperature distribution in the HEMT and in the MTC structure for different gate widths: (a) $5 \mu \mathrm{m}$, (b) $10 \mu \mathrm{m}$, (c) $15 \mu \mathrm{m}$, (d) $20 \mu \mathrm{m}$. Dissipated power in the HEMT was $0.5 \mathrm{~mW}$. From the simulation results follows that the temperature of the MTC structure remains the same. 


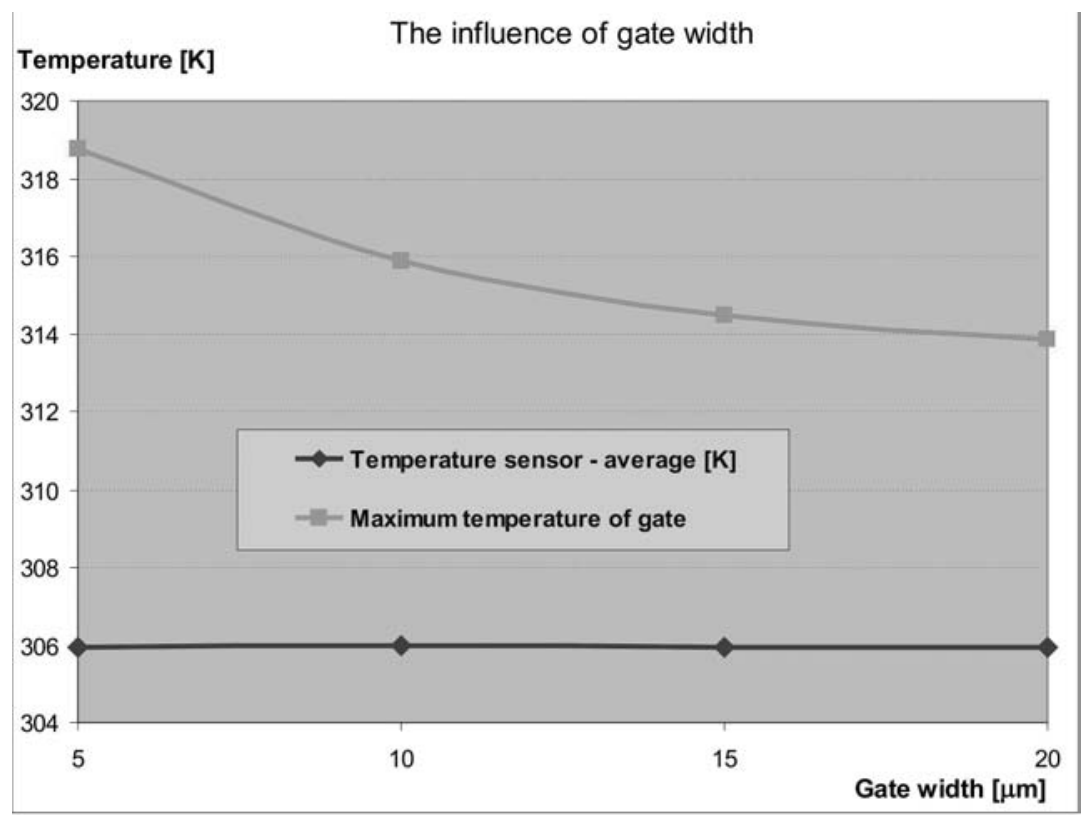

FIGURE 52. Maximal and average temperature-HEMT gate width dependence. Dissipated power in the HEMT was $0.5 \mathrm{~mW}$.

It has been also approved that the temperature sensed by the temperature sensor remained the same. It can be concluded that the HEMT gate width has no influence on the resulting sensitivity, only the maximum temperature changes. In order to minimize the maximum temperature of the sensor it is desirable to increase the HEMT gate width as soon as possible. The dissipated power is then generated in a greater volume. Due to the reduction of the maximum temperature the sensor structure could be used for a wider field of measured power while the sensitivity remains the same.

\section{OPTIMIZATION OF THE DESIGN}

The design criteria to assess the general performance and considerations of the sensor are given below:

- Reduced maximum stress in both the GaAs substrate and the Ti/Au metallization layers, particularly in the heated active area of the MTC device.

- Uniform temperature distribution over the sensing element (meander-like temperature sensor).

- Increased sensitivity (dissipated power-to-temperature conversion).

- Quick time response (change of temperature as a result of changed input power).

According to the above criteria, extensive models of the MTC structure have been designed and numerical simulations have been carried out to evaluate the performance of the sensor. 

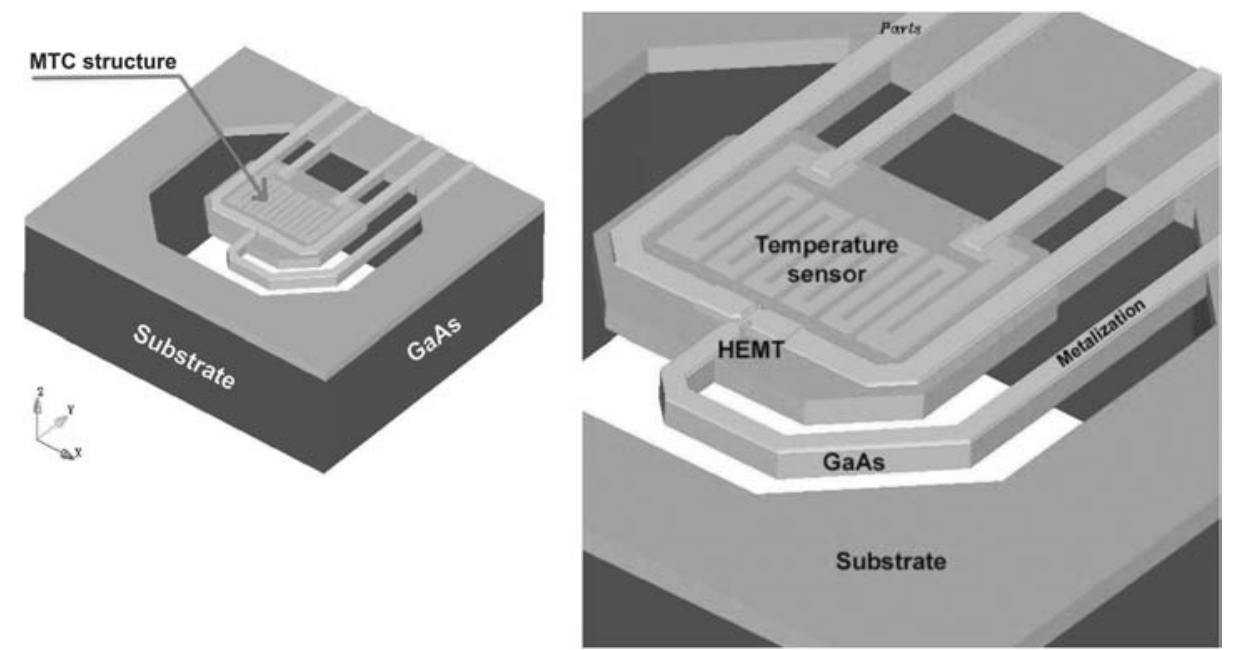

FIGURE 53. 3-D model of the optimized island based MTC device. The scale factor in the $\mathrm{z}$ direction was multiplied by 10 . The polyimide layer is not shown. On the right figure the detail of HEMT and temperature sensor is shown.

A new optimized island structure design reduces the maximal stress caused by temperature changes, minimizes the heat losses caused by too short supplying metallization to the HEMT transistor. The model is depicted in Fig. 53. The gate supplying metallization was led around the island so as to lengthen it as much as possible. Temperature losses are minimized by this solution. Another advantage of such a topology is that all metallizations enter the substrate surface in the same location and there is no other metallization on the opposite site. Mechanical compressions are minimized by this solution, as well.

A steady state temperature analysis has been performed to propose the sensitivity and thermal resistance of the structure. The temperature distribution caused by power dissipation in the heater and the thermal time response as a result of power changes were evaluated by the MemTherm module. For simulation, the input power dissipation in the heater was defined by the heat flux through the HEMT gate area $(10 \mu \mathrm{m} \times 0.5 \mu \mathrm{m})$. We can use this approximation because heat dissipation in the HEMT structure takes place in a very thin conductive layer formed under the gate area. For the thermal analysis problem, the essential boundary conditions are prescribed temperatures. Furthermore, the conductive heat flux and radiation boundary conditions may also be applied. The spatial temperature distribution of the MTCs and steady state heat flux were calculated taking into account the heat transfers to infinity. In the current analysis, according to the application requirement, the fixed thermal boundary is defined for all sidewalls of the GaAs substrate. These sides were kept at room temperature $300 \mathrm{~K}$ while other sides were adiabatic. The CoventorWare simulation manager (SimMan) was used to investigate the influence of the power dissipation in the heater. Plots give a good overall visualization of temperature distribution (Fig. 54) in the island MTC structure. The island is "floating" in the polyimide layer that mechanically and thermally isolates the MTC structure. The polyimide layer is not shown but was considered in the simulation.

The analyses have been performed for both vacuum ambient and non-convective gaseous medium around the MTC structure. The heat losses due to radiation were taken into 


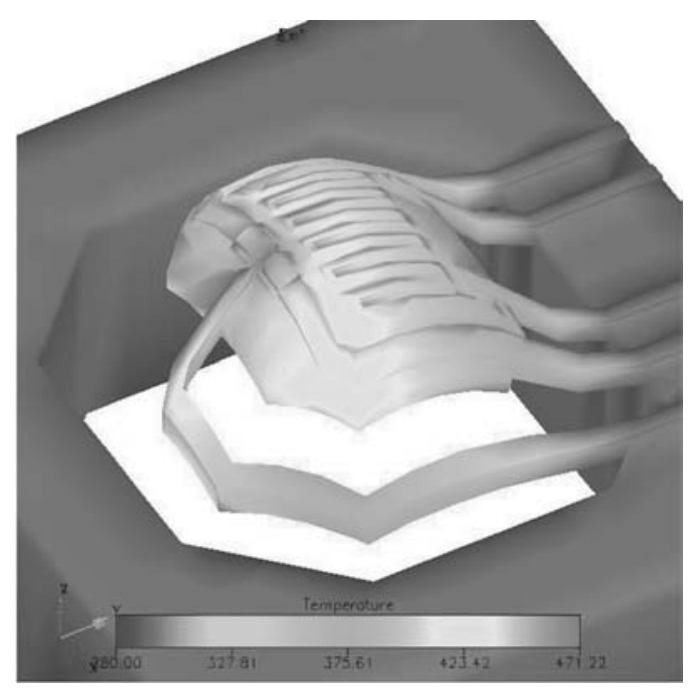

FIGURE 54. 3-D plots of temperature distribution of the island based MTC device. The island is "floating" in polyimide layer that mechanically and thermally isolates MTC structure. Polyimide not shown. The scale factor in the $\mathrm{z}$ direction was multiplied by 10 .

account in the simulation but were found to be negligible. The thermal boundary condition was set the same as for the steady-state analysis. Additionally, the temperature of the MTC body was defined at time $t=0$ to be $300 \mathrm{~K}$. Simulated transient on/off power characteristics for the island structure are depicted in Fig. 55. At the beginning, a power of $1 \mathrm{~mW}$ was switched ON. After $5 \mathrm{~ms}$ the power was switched OFF. The thermal time constant of the island structure arrangement is $0.8 \mathrm{~ms}$. Both, mechanical and thermal boundary conditions

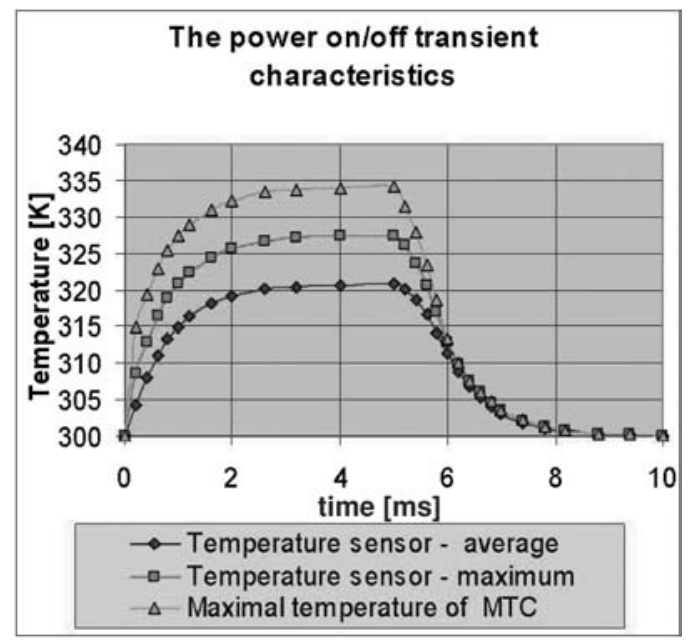

FIGURE 55. The simulated power on/off transient characteristics for island based MTC device for power ON of $1 \mathrm{~mW}$. At the beginning there was power of $1 \mathrm{~mW}$ switched ON. In the time of $5 \mathrm{~ms}$ the power was switched OFF. 

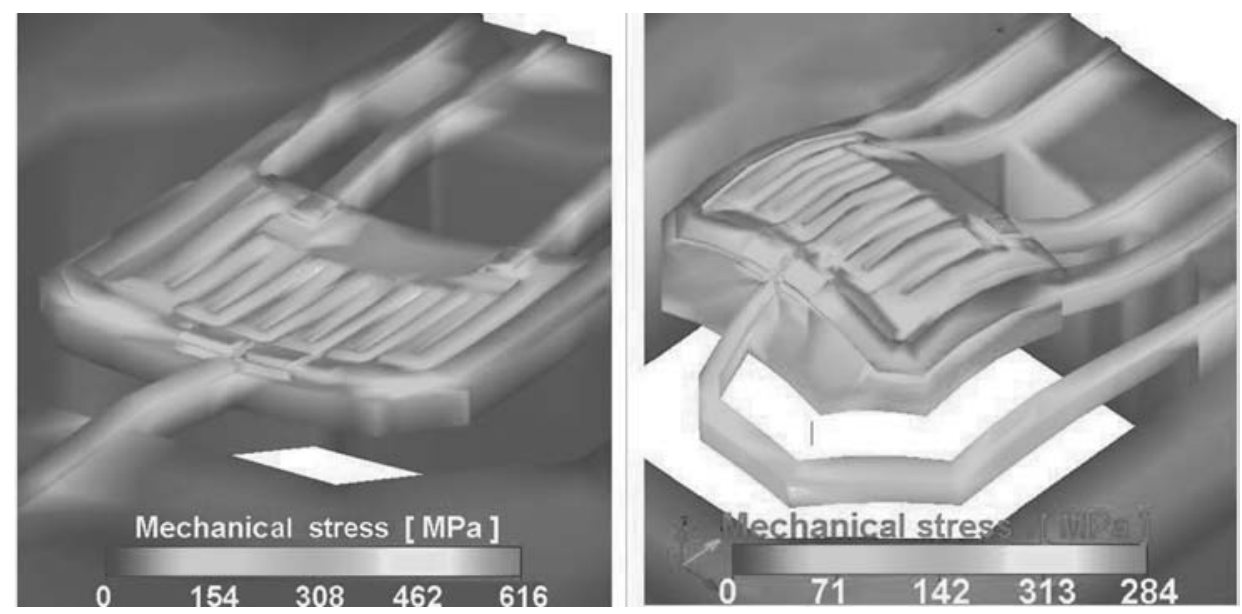

FIGURE 56. Residual stresses and deformation of the island structure caused by heating up with power dissipation of $1 \mathrm{~mW}$ in the heater Left fig.: island structure, Right fig.: optimised island structure, Polyimide layer was investigated in simulation (not visible). Values of stress are in MPa.

were defined for the sidewalls of the GaAs substrate. These sides were kept at room temperature $300 \mathrm{~K}$ while other sides were adiabatic and were set as rigid, i.e., immobile. The initial stress was set in each material according to analytical calculation.

The stress and displacement magnitude were simulated using MemMech simulator. Figure 56 shows the plot of residual stresses and a deformation of the island structure and of the optimized island structure caused by heating. Shading represents the residual stress for $1 \mathrm{~mW}$ power dissipation in the heater. The higher stresses $(616 \mathrm{MPa})$ are located in the place of the meander-shaped PolySi temperature sensor. As seen, the stress is significantly reduced in the optimized island structure design $(284 \mathrm{MPa})$. The scale magnification in $\mathrm{z}$-direction is $10 \times$ and the displacement magnification is $2 \times$.

\section{ACKNOWLEDGEMENTS}

This work was supported, in part, by the NATO SfP Project No.: SfP-974172, CEC COPERNICUS programme-contract No.: CIPA-CT94-0197, Slovak Government contract (No.: 2003 SO 51/03 R06 00/03R06 02-2003), and by the Science and Technology Assistance Agency under the contract No.: APVT-51-032902. The authors would like to thank to Prof. Z. Hatzopoulos from University of Crete for MBE growth of the $\mathrm{AlGaAs} / \mathrm{InGaAs} / \mathrm{GaAs}$ based heterostructures. The authors wish also to thank to research team of the Department of Microelectronics Structures of the Institute of Electrical Engineering, SAS, Bratislava (first of all to Š. Haščík, Ž. Mozolová, M. Grujbár, and I. Benkovič) for participation in the sophisticated MEMS device processing technology. The thanks belongs also to all colleagues from the Department of Electron Beam Lithography of the Institute of Informatics, SAS, Bratislava, for assistance in the device processing technology and fabrication of the required sets of lithographic masks. 


\section{REFERENCES}

1. Leclercq, J.L., Ribas, R.P., Karam J.M., and Viktorovitch P., III-V Micromachined Devices for Microsystems, Microelectronics Journal, 1998;29:613-619.

2. Hjort, K., Soderkvist J., and Jan-Ake Schweitz, Galium Arsenide as a Mechanical Material, J. Micromech. Microeng., 1994;4:1-13.

3. Lalinský, T., Kuzmík, J., Porges, M., Haščík, Š., Mozolová, Ž., and Grňo L., Monolithic GaAs MESFET Power Sensor Microsystem, Electronics Letters, 1995;31:1914-1915.

4. Söderkvist J., A phenomenological Method of Predicting the Performance of Piezoelectric Beams, J. Micromech. Microeng., 1991;1:16-24.

5. Ho, F. and Yamamoto, Y., Piezoresistive-piezoelectric Gallium Arsenide Atomic Force Microscope Cantilever, ERATO Quantum Fluctuation Project, E.L. Ginzton Laboratory, Stanford University, http://wwwsnf.stanford.edu

6. Haščík, Š., Lalinský, T., Kuzmík, J., Porges, M., and Mozolová, Ž., The fabrication of Thin GaAs Cantilever Beams for Power Sensor Microsystem Using RIE, Vacuum, 1996;47:1215-1217.

7. Haščík, Š., Lalinský, T., Mozolovă Ž., and Kuzmík, J., Patterning of Cantilevers for Power Sensor Microsystem, Vacuum, 1998;51:307-309.

8. Karam, J.M., Courtois, B., Holio, M., Leclercq, J.L., and Viktorovitch, P., Collective Fabrication of Gallium Arsenide Based Microsystems, Proceeding SPIE-micromachining and Microfabrication Process Tech. II, 1996, Vol. 2879, pp. 315-326.

9. Ribas, R.P., Leclercq, J.L., Karam, J.M., Courtois, B., and Viktorovitch, P., Bulk Micromachining Characterization of $0.2 \mu \mathrm{m}$ HEMT MMIC Technology for GaAs MEMS Design, Materials Science and Engineering, 1998;B51:267-273.

10. Fobelets, K., Vounckx R., and Borghs, G., A GaAs Pressure Sensor Based on Resonant Tunneling Diodes, J. Micromech. Microeng., 1994;4:123-128.

11. Dehé, A., Fricke, K., Mutanba K., and Hartnagel, H.L., A Piezoresistive GaAs Pressure Sensor with GaAs/AlGaAs Membrane Technology, J. Micromech. Microeng., 1995;5:139-142.

12. Lalinský, T., Burian, E., Mozolová, Ž., Haščík, š., Kuzmík, J., Boháček, P., and Hatzopoulos, Z., The improvment in GaAs Power Sensor Microsystem Technology and Simulation, MICROSIM II-Simulation and Design of Microsystems and Microstructures, in R.A. Adey and Ph. Renaud (Ed.), Computational Mechanics Publications, Southampton, UK, Boston, USA, 1997, pp. 43-51.

13. Lalinský, T., Haščík, Š., Mozolová, Ž., Kuzmík, J., and Hatzopoulos, Z., Power Sensor Microsystem Technology and Characterization, Sensor and Materials, 1998;10:241-250.

14. Lalinský, T., Haščík, Š., Mozolová, Ž., Burian, E., and Držík, M., The Improved Performance of GaAs Micromachined Power Sensor Microsystem, Sensors Actuators A, 1999;76:241-246.

15. Dehé, A., Krozer, V., Fricke, K., Klingbeil, H., Beilenhoff, K., and Hartnagel, H.L., Integrated Microwave Power Sensor, Electronics Letters, 1995;31:2187-2188.

16. Dehé, A., Krozer, V., Chen B., and Hartnagel, H.L., High-sensitivity Microwave Power Sensor for GaAsMMIC Implemention, Electronics Letters, 1996;32:2149-2150.

17. Salzenstein, P., Dupois, O., Hélal, M., Lheurette, E., Vanbésien, O., Mounaix, P., and Lippens, D., Coplanar Waveguides on Dielectric Membranes Micromachined on a GaAs Substrate, Electronics Letters, 1996;32: 821-822.

18. Dehé, A., Fricke, K., and Hartnagel, H.L., Infrared Thermopile Sensor Based on AlGaAs-GaAs Micromachining, Sens. Actuators A, 1995;46-47:432-436.

19. Lalinský, T., Držík, M., Tomáška, M., Krnáč, M., Haščík, Š., Mozolová, Ž., Klasovitý, M., and Kostič, I., Coplanar Waveguides Supported by InGaP and GaAs/AlGaAs Membrane-like Bridges, J. Micromech. Microeng., 2002;12:465-469.

20. Heisig, S. and Oesterschulze, E., Gallium Arsenide Probes for Scanning Near-field Probe Microscopy, Appl. Phys., 1998;A66:S385-S390.

21. Iwata, N., Wakayama, T., and Yamada, S., Establishment of Basic Process to Fabricate full GaAs Cantilever for Scanning Probe Microscope Applications, Sensors and Actuators A, 2004;111;26-31.

22. Lalinský, T., Haščík, Š., Mozolová, Ž., Držík, M., and Hatzopoulos, Z., Micromachined Power Sensor Microsystem, Micromechanics Europe Workshop, Ulvik in Hardanger, Norway, 1998, pp. 139142 
23. Lalinský, T., Držík, M., Haščík, Š., Mozolová, Ž., Kuzmík J., and Hatzopoulos, Z., Study of Bimetallic Effect in a GaAs Cantilever Beam of Power Sensor Microsystem, 2nd International Conference on Advanced Semiconductor Devices and Microsystems, Smolenice Castle, Slovakia, 1998, pp. 331-334.

24. Johansson, S., Ericson, F., and Jan-Ake Schweitz, Influence of Surface Coatings on Elasticity, Residual Stress, and Fracture Properties of Silicon Microelements, J. Appl. Phys., 1989;65:122-128.

25. Lalinský, T., Burian, E., Držík, M., Haščík, Š., Mozolová, Ž., and Kuzmík, J., Thermal Actuation of a GaAs Cantilever Beam, J. Micromech. Microeng., 2000;10:293-298.

26. Lalinský, T., Burian, E., Držík, M., Haščík, Š., Mozolová, Ž., Kuzmík, J., and Hatzopoulos, Z., Performance of GaAs Micromachined Microactuator, Sensors and Actuators, 2000;85:365-370.

27. Brice, J.C., Properties of Gallium Arsenide, 2nd Edition, EMIS Datareviews Series No. 2 (INSPEC (IEE), London, 1990), p. 7.

28. Takahashi, N.S., Properties of Aluminium Gallium Arsenide, in S. Adachi (Ed.), EMIS Datareviews Series No. 7, (INSPEC (IEE), London, 1993), p. 3.

29. Adachi, S., GaAs and Related Materials (Bulk Semiconducting and Superlattice Properties), ISBN 9810219253, World Scientific Publishing Co. Pte. Ltd, 1994

30. Adachi, S., Lattice Thermal Resistivity of III-V Compound Alloys, J. Appl. Phys. 1983;54:1844-1848.

31. Adachi, S., GaAs, AlAs and $\mathrm{Al}_{\mathrm{x}} \mathrm{Ga}_{1-\mathrm{x}}$ As Material Parameters for Use in Research and Device Applications, J. Appl. Phys. 1985;58:R1-29.

32. Walkey, D.J., Smy, T.J., Macelwee, T., and Maliepaarad, M., Compact Representation of Temperature and Power Dependence of Thermal Resistance in Si, InP and GaAs Substrate Devices Using Linear Models, Solid-State Electronics, 2002;46:819-826.

33. Hjort, K., Sacrificial Etching of III-V Compounds for Micromechanical Devices J. Micromech. Microeng., 1996;6:370-375.

34. Collins, S.D., Etch Stop Techniques for Micromachining, J. Electrochemical Society, 1997;144:2242-2262.

35. Fricke, K., Würf, J., Miao, J., Dehé, A., Rück, D., and Hartnagel, H.L., Fabrication of Microstructures for Integrated Sensors on GaAs, J. Micromech. Microeng., 1993;3:131-134.

36. Miao, J., Hartnagel, H.L., Rück, D., and Fricke, K., The Use of Ion Implantation for Micromachining GaAs for Sensor Application, Sensors and Actuators A, 1995;46-47:30-34.

37. Uenishi, Y., Tanaka, H., and Ukita, H., Characterization of AlGaAs Microstructure Fabricated by AlGaAs/GaAs Micromachining, IEEE Trans. On Electron Devices, 1994;41:1778-1783.

38. Chong, N., Srinivas, T.A.S., and Ahmed, H., Performance of GaAs Microbridge Thermocouple Infrared Detectors, Journal of Microelectromechanical Systems, 1997;6:136-141.

39. Ribas, R.P., Maskless Front-Side Bulk Micromachining Compatible to Standard GaAs IC Technology, Thesis, TIMA-CMP, Grenoble Cedex, France.

40. TIMA-Circuits Multi-Projects (CMP) Laboratory: $0.2 \mu \mathrm{m}$ pHEMT GaAs E/D from OMMIC, Compatible Front-side Bulk Micromachining, Grenoble Cedex, France.

41. Lalinský, T., Držík, M., Matay, L., Kostič, I., Mozolová, Ž., Haščík, Š., and Krajcer, A., InGaP/Polyimide Membrane-like Bridges Fully Compatible with InGaP/InGaAs/GaAs Based HFETs, Proceedings of the International MEMS Workshop-iMEMS, Singapore, July 4-6, 2001, pp. 362-368, to be Published Also in Materials \& Process Integration for MEMS, Edited by Francis E.H. Tay, Singapore, ISBN 1-4020-7175-2, 2002, pp. 53-75.

42. Lalinský, T., Haščík, Š., Mozolová, Ž., Burian, E., Tomáška, M., Krnáč, M., Držík, M., Škriniarová, J., Kostič, I., and Matay, L., Mechanically Fixed and Thermally Isolated Micromechanical Structures for GaAs Heterostructure Based MEMS Devices, Proceedings of the International Symposium on Microelectronics, Colorado, September 4-6 2002, pp. 87-92 - to be Published Also in Microelectronics International, January 20, 2003, pp. 43-47.

43. Lalinský, T., Hotový, I., Haščík, Š., Mozolová, Ž., Kuzmík, J., and Pogany, D., Thin Film Resistance Temperature Sensors on GaAs, Proceeding of ASDAM' 98 Conference, Smolenice Castle, Slovakia, 1998, pp. 243-246.

44. Lalinský, T., Hrkút, P., Mozolová, Ž., Kovačik, T., and Krajcer, A., Thermal Performance and Stability of Poly Si/Pt(Ni) Thin Film Temperature Sensors on GaAs, Proceedings of the 11th International Conference on Solid-State Sensors and Actuators, Munich, June 10-14, 2001, pp. 1496-1499.

45. Fricke, K., Hartnagel, H.L., Ritter, S., and Würfl, J., Micromechanically Structurized Sensors on GaAs: an Integrated Anemometer, Microelectronics Engineering, 1992;19:195-198. 
46. Fricke, K., A Micromachined Mass-flow Sensor With Integrated Electronics on GaAs, Sensors and Actuators A, 1994;45:91-94.

47. Dehé, A. and Hartnagel, H.L., Free-standing AlGaAs Thermopilesfor Improved Infrared Sensor Design, IEEE Trans. on Electron Devices, 1996;43:1193-1199.

48. Hava, S. and Hunsperger, R., Thermoelectric Properties of $\mathrm{Al}_{\mathrm{x}} \mathrm{Ga}_{1-\mathrm{x}} \mathrm{As}$, J. of Appl. Phys., 1985;57:53305335.

49. Pogany, D., Seliger, N., Lalinský, T., Kuzmík, J., Habaš, P., Hrkút, P., and Gornik, E., Study of Thermal Effects in GaAs Micromachined Power Sensor Microsystems by An Optical Interferometer Technique, Microelectronics Journal, 1998;29:191-198.

50. Burian, E., Pogany, D., Lalinský, T., Seliger, N., and Gornik, E., Thermal Simulation and Characterization of GaAs Micromachined Power Sensor Microsystems, Sensors Actuators A, 1998;68:372-377.

51. Lalinský, T., Haščík, Š., Mozolová, Ž., Držík, M., and Hatzopoulos, Z., The Improved Performance of GaAs Micromachined Power Sensor Microsystem, Proceedings of the 12th European Conference on Solid-State Transducers-EUROSENSORS XII, Southampton, September 13-16, 1998, pp. 739-742.

52. Dehé, A., Peerlings, J., Pfeiffer, J., Riemenschneider, R., Vogt, A., Streubel, K., Künzel, H., Meissner, P., and Hartnagel, H.L., III-V Compound Semiconductor Micromachined Actuator for Long Resonator Tunable Fabry-Pérot Detector, Sensors and Actuators A, 1998;68:365-371.

53. Lalinský, T., Krnáč, M., Držík, M., Haščík, Š., and Mozolová, Ž., Micromechanical Thermal Converter Device Based on Polyimide-fixed Island Structure, The 14th Micromechanics Europe Workshop-MME03, Delft, November 2-4, 2003, pp. 45-48.

54. Lalinský, T., Držík, M., Chlpík, J., Krnáč, M., Haščík, Š., and Mozolová, Ž., Thermo-Mechanical Characterization of Micromachined GaAs Based Thermal Converter Using Contactless Optical Methods, In: Proceedings of EUROSENSORS XIII Conference, Roma September 12-15, 2004, p. 239.

55. Microwave Monolithic Integrated Transmitted Power Sensors and Their Industrial and Metrological Applications, NATO SfP project No.: SfP-974172.

56. Born, M. and Wolf, E., Principles of Optics, Pergamon Press, New York, 1975.

57. Ohring, M., The Material Science of Thin Films, Academic Press, New York, 1992.

58. Bertollotti, M., Bogdanov, V., Ferrari, A., Jascow, A., Nazarova, N., Pikhtin, A., and Schirone, L., Temperature Dependence of the Refractive Index in Semiconductors, J.Optical Soc. of America, 1990;7:918-922.

59. McCaulley, J.A., Donnelly, V.M., Vernon, M., and Taha, I. Temperature Dependence of the Near-infrared Refractive Index of Silicon, Gallium Arsenide, and Indium Phosphide, Physical Review B, 1994;49:74087017.

60. Tiziani, H.J., Optical Metrology of Engineering Surfaces-Scope and Trends, in P.K. Rastogi (Ed.), Optical Measurement Techniques and Applications, Artech House, Boston, 1997, pp. 15-49.

61. Sheppard, C.J.R. and Roy, M., Low-coherence Interference Microscopy, in P. Torok and F.-J. Kao (Eds.), Optical Imaging and Microscopy, Springer, Berlin, 2003.

62. Davis, C.Q. and Freeman, D.M., Using a Light Microscope to Measure Motions with Nanometer Accuracy, Optical Engineering, 1998;37:1299-304.

63. Sheppard, C.J.R., Roy, M., and Sharma, M.D., Image Formation in Low-coherence and Confocal Interference Microscopes, Applied Optics, 2004;43:1491-1502.

64. Bosseboef, A. and Petitgrand, S., Characterization of the Static and Dynamic Behaviour of M(O)EMS by Optical Techniques: Status and Trends, Proceeding MME'02, Sinaia, Romania, 2002, pp. 229-241.

65. Wilson, T., Confocal Microscopy, Academic Press, New York, 1990.

66. PL $\mu$ : Confocal Imaging Profiler, The Innovative Concept for Non-contact 3D, http://www.atos-online.de.

67. Jordan, H.J., Brodmann, R., Valentin, J., and Grigat, M., Confocal White Light Microscopy, http://www. nanofocus-inc.com $/ \mathrm{html}$.

68. Vlassak, J.J. and Nix, W.D., A New Bulge Test Technique for The Determination of Young's Modulus and Poisson's Ratio of Thin Films, J. Mater. Res., 1992;7:3242-3249.

69. FLATSCAN 200-Automated 2D/3D-Measurement System for Thin Film Stress and Surface Curvature, http://www.oeg-messtechnik.de.

70. KLA Tencor FLX-2320-S Film Stress Measurement Systém, http://www.lot-oriel.com/pdf/all/kla_ filmstress.pdf

71. Drzik, M., Vanek, O., and Kempny, M., Silicon Wafers Deformation Measurement by Using Optical Methods, Proceeding of 41st International Science Colloquium, 1996, Vol. 1, pp. 597-602. 
72. Nemcsics, A., Szabo, J., Gurban, S., and Csontos, L., Mechanical Study of the Strained $\operatorname{In}_{\mathrm{X}} \mathrm{Ga}_{1-\mathrm{x}} \mathrm{As} / \mathrm{GaAs}$ Heterostructures, in J. Novak and A. Schlachetzki (Eds.), Heterostructure Epitaxy and Devices, Kluwer Academic Publ., 1996, pp. 103-106.

73. Drzik, M., Butschke, J., Fallmann, W., Haugeneder, E., and Loeschner, H., Optical Measurement of Stress in Thin Membranes, Proceeding of 26th IMEKO World Congress, Vienna, CD-ROM, 2000.

74. Levtchenko,V.M. and Gulidov, D.N., Mechanical Treatment of Semiinsulator (SI) GaAs (100) and its Effect on Geometrical and Electro-physical Parameters, Proceeding of 5th Science and Business Conference SILICON`96 Vol. 2 Roznov p. Radhostem, 1996, pp. 278-292.

75. Gunnars, J. and Wiklund, U., Determination of Growth-induced Strain and Thermoelastic Properties of Coatings by Curvature Measurements, Materials Science and Engineering, 2002; Vol. A336, pp. 7-21.

76. Chen, K.-S. and Ou, K.-S., Modification of Curvature-based Thin-film Residual Stress Measurement for MEMS Applications, J. Micromech. Microeng., 2002;12:917-924.

77. Fang, W. and Wickert, J.A., Comments on Measuring Thin-film Stresses Using Bi-layer Micromachined Beams, J. Micromech. Microeng., 1995;5:276-281.

78. Yang, J.C.S., Prevention of Thermal Bending of Multilayered Beams and Plates, Experimental Mechanics $1975 ; 15: 418-423$.

79. Suhir, E., Stresses in Bi-metal Thermostats, J. Applied Mechanics, 1986;53:657-660.

80. Lavitska, E., Thermal Strain and Its Influence on Characterization of Semiconductor Elements, Proceeding of 5th NEXUSPAN Workshop on Thermal Aspects in Microsystem Technology, Budapest, 1998; pp. $115-120$.

81. Zhang, X., Ghodssi, R., Chen, K.-S., Ayon, A.A., and Spearing, S.M., Residual Stress Characterization of Thick PECVD TEOS Film For Power MEMS Applications, Proceeding of Solid-State Sensor and Actuator Workshop, Hilton Head Is, SC, 2000, pp. 1-4.

82. Castellini, P., Revel, G.M., and Scalise, L., Application of Lasers for Non-contact Excitation and Measurement of Vibration, Proceeding SPIE - 4th Int. Conference on Vibration Measurements by Laser Technique, 2000, Vol. 4072, pp. 280-291.

83. Jia, W. and Enyao, Zh., Applications of Laser Vibrometer in Vibration Measurement of Microstructures, Proceeding SPIE, 1996;2868:318-325.

84. Petitgrand, S., Yahiaoui, R., Bosseboef, A., and Danaie, K., Quantitative Time-average Microscopic Interferometry for Micromechanical and Art Conservation, Proceeding SPIE, 2001;4400:51-60.

85. Abdel-Rahman, E.M., Younis, M.I., and Nayfeh, A.H., Characterization of the Mechanical Behavior of An Electrically Actuated Microbeam, J. Micromech. Microeng., 2002;12:759-766.

86. Lawrence, E.M. and Speller, K.E., MEMS Characterization Using Laser Doppler Vibrometry, Proceeding of SPIE, 2002;4980:323-329.

87. Kopystyski P., Obermayer H., Delfs H., Hohenester W., and Loser A., Silicon RF Power Sensor from DC to Microvawe, Microsystems Technologies 90, Springer, Berlin, 1990, p. 605.

88. Jaeggy, Baltes, H., and Moser, D., Thermoelectric AC Power Sensor by CMOS Technology, IEEE Electron Device Letters, 1992;13:366.

89. Senturia, S.D., Microsystem Design, Kluwer Academic Publisher, book, 2001.

90. Elwensoekk, M. and Wiegerink, R., Mechanical Mikrosensors, Springer, book, 2000.

91. Tai-Ran Hsu, MEMS and Microsystems Design and Manufacture, Mc Graw Hill, book, 2001.

92. MEMCAD—User Manual.

93. Georgakilas, A., Lalinský, T., Husák, M., and Grňo, L., Microwave Monilitic Integrated Transmitted Power Sensor and Their Industrial and Metrological Applications, NATO SfP Project No.: SfP-974172, 1999.

94. Jakovenko, J. and Husák, M., Thermo-mechanical Simulation of GaAs Power Sensor Microsystem, Poster 2001 Conference, Prague, Czech Republic, 2001.

95. http://k315.feld.cvut.cz/download/men/COUP4.pdf.

96. Kim, Y. and Allen, G., Measurement of Mechanical Properties of Polyimide Films Using Micromachined Resonant String Structures, Transactions on Components and Packaging Technology, 1999;22(2).

97. Rizk, J., Chaiban, E., and Rebeiz, G., Steady State Thermal Analysis and High-Power Reliability Considerations of RF MEMS Capacitive Switches, Proceedings of IMS-02, 2002.

98. http://heat.feld.cvut.cz/vyuka/2/index.html.

99. Jakovenko, J., Husak, M., and Lalinsky, T., Design and Simulation of Micromechanical Thermal Converter for RF Power Sensor Microsystem, Microelectronics Reliability, 2004;141-148. 
100. Jakovenko, J., Husak, M., and Lalinsky, T., Design and Simulation of The GaAs Micromechanical Thermal Converter for Microwave Transmitted Power Sensor, Proceedings of Nanotechnology Conference, San Francisco, 2003, pp. 332-335.

101. Jakovenko, J., Husak, M., and Lalinsky, T., Modeling of Thermally Isolated Micromechanical Thermo Converter, The 17th European Conference on Solid-State Transducers, EUROSENSORS XVII, Guimarăes 2003, pp. 316-317.

102. Jakovenko, J., Husák, M., and Lalinsky, T., Thermo-mechanical Simulation and Modeling of RF Power Sensor Microsystem, IMAPS Proceedings 2002, 35th International Symposium on Microelectronics, Denver, Colorado, ISBN 0-930815-66-1, 2002, pp. 886-891.

103. Jakovenko, Husák, M., and Lalinsky, T., Thermo-mechanical Simulations of GaAs Based Microwave Power Sensor Microsystem, EUROSENSORS XVI Book of Abstracts, Prague, Czech Republic, ISBN 80-01-02576-4 2002, pp. 63-64.

104. Jakovenko, J. and Husák, M., MEMCAD Thermal Simulation of GaAs Based Membrane Bridge, Proceedings of 12th Micromechanics Europe Workshop (MME'01), Cork, 2001, pp. 225-228.

105. Incropera, F.P. and DeWitt, D.P., Fundamentals of Heat and Mass Transfer, JohnWiley \& Sons, New York, 2002 . 